\title{
Scattering of the Low-Mode Internal Tide at the Line Islands Ridge
}

by

Maha Niametullah Haji

B.S. Mechanical Engineering, B.A. Applied Mathematics (2012)

University of California, Berkeley

Submitted in partial fulfillment of the requirements for the degree of

Master of Science in Oceanographic Engineering

at the

MASSACHUSETTS INSTITUTE OF TECHNOLOGY

and the

WOODS HOLE OCEANOGRAPHIC INSTITUTION

February 2015

(c) 2015 Maha N. Haji. All rights reserved.

The author hereby grants to MIT and WHOI permission to reproduce and to distribute publicly paper and electronic copies of this thesis document in whole or in part in any medium now known or hereafter created.

Author

Departments of Mechanical Engineering, MIT \& Applied Ocean Science and Engineering, WHOI

January 16, 2015

Certified by . .

Thomas Peacock

Associate Professor of Mechanical Engineering, MIT Thesis Supervisor

Accepted by

David Hardt

Chair, Department Committee on Graduate Studies, MIT

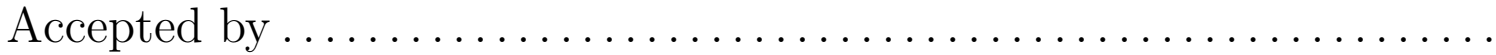

Henrik Schmidt

Chair, Joint Committee for Applied Ocean Science and Engineering, MIT 


\title{
Scattering of the Low-Mode Internal Tide at the Line Islands Ridge
}

by

\author{
Maha Niametullah Haji
}

\author{
Submitted to the Departments of Mechanical Engineering, MIT \\ \& Applied Ocean Science and Engineering, WHOI \\ on January 16, 2015, in partial fulfillment of the \\ requirements for the degree of \\ Master of Science in Oceanographic Engineering
}

\begin{abstract}
The scattering of low-mode internal tides by ocean-floor topography is extensively studied through analytical models and field observations at the Line Islands Ridge (LIR). An existing Green function method is utilized to examine the generation of internal tides by idealized topographic shapes as well as realistic transects of the LIR. The method is also applied to examine the scattering of a mode- 1 internal tide at these topographies to determine the relative high mode energy flux due to generated and scattered internal tides at the realistic transects.

A method of determining the modal content of an internal wave field is advanced to account for arbitrary stratification and rotation. It is then adjusted to allow for image loss as is common to oceanographic studies. Its performance is compared to the existing regression method widely used by oceanographers to determine the modal content of internal tides. The results from this comparison are used to inform the analysis of the field observations.

This thesis concludes by examining the modal content of the LIR as determined from measurements taken during the 150-day EXperiment on Internal Tide Scattering (EXITS) NSF field study. Motivated by satellite altimetry data and three-dimensional numerical model studies, the EXITS cruise sought to observe the internal tide scattering process in the ocean for the first time. The data from three moorings equipped with moored profilers, spanning total depths of $3000-5000 \mathrm{~m}$ is analyzed to determine the modal content of the southward propagating internal tide before and after it encounters the ridge for evidence of topographic scattering.
\end{abstract}

Thesis Supervisor: Thomas Peacock

Title: Associate Professor of Mechanical Engineering, MIT 


\section{Acknowledgments}

Sincere thanks to my thesis advisor, Professor Thomas Peacock, for his support and guidance throughout this project, starting from before I even knew what internal waves were. Most of all, thank you for giving me the opportunity to travel and meet some amazing scientists in the field. I could not have become the researcher I am today without your help.

Thank you to the National Science Foundation for their generious founding for this project. Any opinion, findings, and conclusions or recommendations expressed in this material are those of the author and do not necessarily reflect the views of the National Science Foundation.

Thank you to the many ENDLab members I have had the pleasure of working with during my time at MIT: Michael Allshouse, Séverine Atis, Margaux Filippi, Sasan Ghaemsaidi, Nils Holzenberger, Mathieu Leclair, Mathieu Mercier, Zaim Ouazzani and honorary member, Hussain Karimi. Your friendship, advice, and discussions, especially of the oceanographic sort, have helped me tremendously. Thanks to Ray Hardin, Leslie Regan, Joan Kravit, Ronni Schwartz, Leanora Fraser, and Julia Westwater for their unending help with all administrative issues.

Many thanks to Professor Manikandan Mathur for helping me understand and implement the Green function method utilized in Chapter 2. I would also like to thank Professors Shaun Johnston and Glenn Carter for their collaboration on the work presented in Chapter 4. It was thanks to their willingness to teach that I was able to understand the methods of physical oceanography. Additionally, I would like to thank Professors John Toole and Matthew Alford, Leah Trafford, and Andy Pickering for all their patience and help in learning how to process the MMP field data. Thanks to Zhongxiang Zhao for providing an MMP toolbox and helpful instructions without which I would not have been able to present results of Chapter 4

Thank you to my friends who have been there for me and have made the past

few years enjoyable: Jay Brett, Pierre Claver, Giulia DeSalvo, Fayzan Gowani, Julia Hopkins, Brie Robertori, James Schulmeister, Stephanie Steele, and Sarah Thornton. 
Special thanks to Monica Orta, for always being a kind shoulder to lean on when times got rough.

Many, many thanks to Sasan for being there for me throughout the process. Your support, knowledge, and motivation have helped me grow in ways I did not know were possible. I could not have done this without you.

Most of all, I would like to thank my mother, father, and brother. Thank you for your never-ending faith in my abilities, encouragement, and understanding throughout my life. I would not be the person I am today without you. 


\section{Contents}

$\begin{array}{lll}1 & \text { Introduction } & 13\end{array}$

1.1 Background $\ldots \ldots \ldots \ldots \ldots$

1.2 Governing equations $\ldots \ldots \ldots \ldots \ldots \ldots$

1.3 Internal wave modes $\ldots \ldots \ldots \ldots$

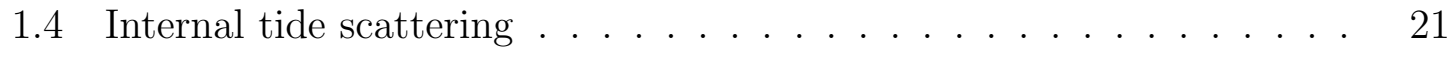

1.5 Thesis overview . . . . . . . . . . . . . . . . . . . . . . . . 24

$\begin{array}{lll}2 & \text { Analytical Model } & 25\end{array}$

2.1 Introduction . . . . . . . . . . . . . . . . . . . . . . 25

2.2 Theory $\ldots \ldots \ldots \ldots \ldots \ldots$

2.3 Idealized Gaussian ridge $\ldots \ldots \ldots \ldots \ldots$

2.3 .1 Generation results . . . . . . . . . . . . . . . . 35

$2.3 .2 \quad$ Scattering results $\ldots \ldots \ldots \ldots \ldots$

$2.3 .3 \quad$ Generation vs. Scattering $\ldots \ldots \ldots \ldots$. . . . . . . . . 41

2.4 Line Islands Ridge transects $\ldots \ldots \ldots \ldots \ldots$. . . . . . . . . . . 42

2.4 .1 Generation results . . . . . . . . . . . . . . . 45

2.4 .2 Scattering results $\ldots \ldots \ldots \ldots \ldots$

2.5 Discussion and Conclusions $\ldots \ldots \ldots \ldots \ldots$

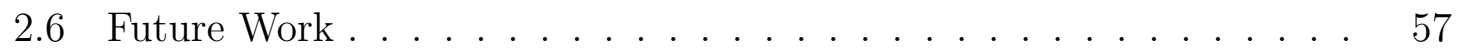

3 Modal Decomposition of Internal Wave Fields 61

3.1 Introduction . . . . . . . . . . . . . . . . . . . . . . . . . 61

3.2 Projection method $\ldots \ldots \ldots \ldots \ldots$ 
3.3 Regression method $\ldots \ldots \ldots \ldots$. . . . . . . . . . . . . 68

3.4 Results . . . . . . . . . . . . . . . . . . . . . . . . . . . . . . . . 69

$3.4 .1 \quad$ Full vertical domain $\ldots \ldots \ldots \ldots$. . . . . . . . . . . 71

3.4 .2 Incomplete vertical domain $\ldots \ldots \ldots \ldots \ldots . \ldots \ldots$

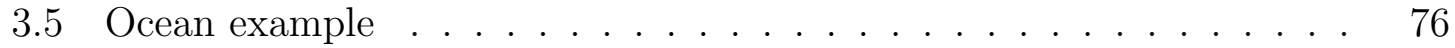

3.6 Determining $M \ldots \ldots \ldots \ldots \ldots$

\begin{tabular}{|lll}
\hline 4 & Field Experiment Results & 81
\end{tabular}

4.1 Introduction . . . . . . . . . . . . . . . . . . . . . . . . . 81

4.2 Data and methods $\ldots \ldots \ldots \ldots$. . . . . . . . . . . . . . . 82

$4.2 .1 \quad$ The EXITS field study $\ldots \ldots \ldots \ldots$

4.2 .2 Profiling mooring instruments . . . . . . . . . . . . . . 84

$4.2 .3 \quad$ Bandpass filtering $\ldots \ldots \ldots \ldots \ldots$

$4.2 .4 \quad$ Vertical displacement . . . . . . . . . . . . . . . . . 86

4.2 .5 Modal decomposition . . . . . . . . . . . . . . . . 86

$4.2 .6 \quad$ Pressure, energy, and energy flux . . . . . . . . . . . . 90

4.3 Mooring-observed internal tides . . . . . . . . . . . . . . . . . . 91

$4.3 .1 \quad$ Interntal tide at mooring $\mathrm{U} \ldots \ldots \ldots$

4.3 .2 Interntal tide at mooring $\mathrm{N} \ldots \ldots \ldots$

$4.3 .3 \quad$ Interntal tide at mooring S . . . . . . . . . . . . . . . 97

4.3 .4 Variations between moorings . . . . . . . . . . . . . . . . 99

4.4 Uncertainties $\ldots \ldots \ldots \ldots$. . . . . . . . . . . . . . . . . 100

4.5 Conclusion $\ldots \ldots \ldots \ldots \ldots \ldots \ldots$

$\begin{array}{lll}5 \text { Conclusions } & 105\end{array}$ 


\section{List of Figures}

1-1 Energy loss from the barotropic tide calculated by Egbert and Ray (Egbert \& Ray, 2001) from satellite altimetry data. . . . . . . . . . . 14

1-2 The first three modes, $\phi_{n}(z)$, for $(a)$ a uniform stratification profile, $N(z)=N$, and $(b)$ a nonuniform stratification profile, $N(z)$, characteristic of the ocean. In $(b)$, the vertical profiles indicate a concentration of amplitude and highest gradients in the vicinity of the peak of $N(z)$, the pycnocline. . . . . . . . . . . . . . . . . . 20

1-3 The global tidal energy budget depicting the energy transfers from the surface tides to mixing scales (modified from Munk \& Wunsch (1998)). More than $50 \%$ of the energy lost by the surface tide through boundary interactions is suggested to end up in internal waves that radiate away from the generation site. . . . . . . . . . . . . . . . . 21

1-4 Altimeter sea surface height modulations due to the $(a)$ mode-1 and $(b)$ mode-2 $M_{2}$ internal tide. Grey shading shows bathymetry (Johnston et al. [2003)] . . . . . . . . . . . . . . . . . . . 22

1-5 The horizontal velocity field $u(x, z, t)$ at an arbitrary instant in time for mode-1 internal tide (incident from the left) scattering in a nonuniform stratification by a (a) subcritical $(\epsilon=0.5)$ and (b) supercritical $(\epsilon=2)$ Gaussian ridge with $h^{*}=0.5$ (calculated using the Green function model) (Mathur et al.]2014). . . . . . . . . . . . . . . . . 23

2-1 Problem geometry showing the boundaries and point-source distribution. 27

$2-2 \quad$ iTides Graphical User Interface (GUI). . . . . . . . . . . . . . . . . . 33 
2-3 The $(a)$ nonuniform stratification and $(b)$ topography of the idealized Gaussian ridge representation of a characteristic transect of the Line Islands Ridge. In $(b)$ the dashed lines MN and MS indicate effective locations of mooring $\mathrm{N}$ and $\mathrm{S}$ from the EXITS study, respectively. . . 34

2-4 Normalized horizontal velocity field $\left(u(x, z, t) / u_{0}\right)$ at an arbitrary instant in time generated at the idealized Gaussian ridge depicted in |figure $2-3$ [ . . . . . . . . . . . . . . . . . . . . 35

2-5 (a) Modal decomposition of the leftward $\left(F_{n}^{R}\right)$ and rightward $\left(F_{n}^{T}\right)$ propagating energy flux and $(b)$ the local depth-integrated, time-averaged, across-ridge energy flux of the generated internal wave field, both normalized by the total energy flux. In $(b)$, dashed lines MN and MS correspond to the effective locations of mooring $\mathrm{N}$ and $\mathrm{S}$ of the EXITS - study, respectively. . . . . . . . . . . . . . . 36

2-6 Modal decomposition of the net energy flux of the generated internal \begin{tabular}{|c|}
\hline tide before, after, and at locations MN and MS on the topography of \\
\hline
\end{tabular} \begin{tabular}{|c|c|c|}
\hline the Gaussian ridge shown in figure & $2-3$ & normalized by the total energy \\
\hline
\end{tabular} \begin{tabular}{|c|}
\hline flux. The profiles are decomposed using the $(a)$ local depth and $(b)$ \\
\hline
\end{tabular} far-field depth. The net energy flux in the far-field before the topography $\left(F_{n}^{R}\right)$ and after the topography $\left(F_{n}^{T}\right)$ are also plotted, and should roughly agree with the modal decomposition of the net energy flux at

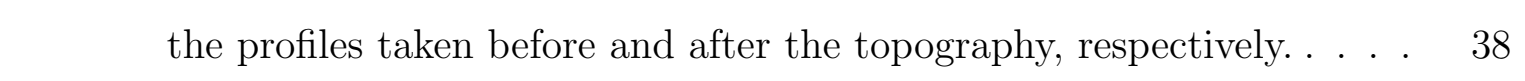

2-7 $\quad$ Normalized incident-plus-scattered horizontal velocity field $\left(u(x, z, t) / u_{0}\right)$ at an arbitrary instant in time for mode-1 (incident from the left) scat-

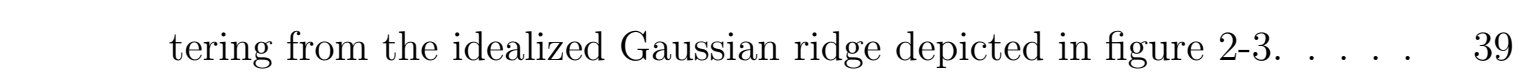

$2-8 \quad(a)$ Modal decomposition of the transmitted $\left(F_{n}^{T}\right)$ and reflected $\left(F_{n}^{R}\right)$ \begin{tabular}{l}
\hline energy flux and $(b)$ the local depth-integrated, time-averaged, across- \\
\hline ridge energy flux of the scattered internal wave field, both normalized \\
\hline by the incident mode-1 energy flux. In $(b)$, dashed lines MN and MS \\
\hline correspond to the locations of mooring N and S of the EXITS study, \\
\hline respectively. . . . . . . . . . . . . . . . . . . . . . . . . . . . . . 39
\end{tabular} 
2-9 Modal decomposition of the net energy flux of the incident-plus-scattered internal tide before, after, and at locations MN and MS on the topography of the Gaussian ridge shown in figure $|2-3|$ normalized by the incident energy flux. The profiles are decomposed using the $(a)$ local depth and (b) far-field depth. The energy flux before the topography $\left(F_{n}^{R+i}\right)$ and after the topography $\left(F_{n}^{T}\right)$ are also plotted, and should roughly agree with the modal decomposition of the net energy flux at the profiles taken before and after the topography, respectively. . . . .

2-10 A contour plot of the topography of the Line Islands Ridge, indicating the locations of the three internal wave observation moorings from the EXITS cruise: mooring U, mooring N, and mooring S. The scattering of a mode-1 internal tide and internal tide generation by barotropic forcing is investigated for transects between moorings $\mathrm{U}$ and $\mathrm{N}$ (U$\mathrm{N}$ transect) and moorings $\mathrm{N}$ and $\mathrm{S}$ (N-S transect), indicated by the dashed lines. . . . . . . . . . . . . . . . . . . . . . . .

2-11 The $(a)$ stratification and (b) topography of the U-N transect of the \begin{tabular}{|l|l|l|l|}
\hline Line Islands Ridge shown in figure & $2-10$ & In $(b)$ the dashed lines MU and
\end{tabular} $\mathrm{MN}$ indicate effective locations of mooring $\mathrm{U}$ and $\mathrm{N}$ from the EXITS study, respectively. . . . . . . . . . . . . . . . . . . . . . . 44

2-12 The (a) stratification and (b) topography of the N-S transect of the \begin{tabular}{|c|c|c|c|}
\hline Line Islands Ridge shown in figure & $2-10$. & In $(b)$ the dashed lines MN
\end{tabular} and MS indicate effective locations of mooring $\mathrm{N}$ and $\mathrm{S}$ from the EXITS study, respectively. . . . . . . . . . . . . . . . . . . . . . 44

2-13 Normalized horizontal velocity field $\left(u(x, z, t) / u_{0}\right)$ of the internal tide at an arbitrary instant in time generated at the U-N transect shown in

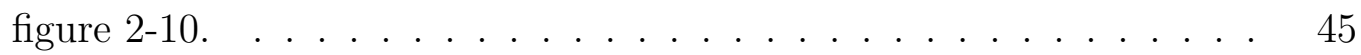


2-14 (a) Modal decomposition of the leftward $\left(F_{n}^{R}\right)$ and rightward $\left(F_{n}^{T}\right)$ propagating energy flux and (b) the local depth-integrated, time-averaged, across-ridge energy flux of the internal tide generated at the U-N transect shown in figure $2-10$, Both are normalized by the total energy flux. In (b), dashed lines MU and MN correspond to the locations of mooring $\mathrm{U}$ and $\mathrm{N}$ of the EXITS study, respectively. . . . . . . . . . . 46

2-15 Modal decomposition of the net energy flux of the generated internal tide before, after, and at locations MU and MN on the topography of the U-N transect shown in figure $2-11 \mid$ normalized by the total energy flux. The profiles are decomposed using the $(a)$ local depth and $(b)$ far-field depth. The net energy flux in the far-field before the topography $\left(F_{n}^{R}\right)$ and after the topography $\left(F_{n}^{T}\right)$ are also plotted, and should roughly agree with the modal decomposition of the net energy flux at the profiles taken before and after the topography, respectively. . . . .

2-16 Normalized horizontal velocity field $\left(u(x, z, t) / u_{0}\right)$ at an arbitrary instant in time generated at the N-S transect shown in figure $2-10$. . . .

2-17 (a) Modal decomposition of the leftward $\left(F_{n}^{R}\right)$ and rightward $\left(F_{n}^{T}\right)$ propagating energy flux and (b) the local depth-integrated, time-averaged, across-ridge energy flux of the internal tide generated at the N-S transect shown in figure $2-10$. Both are normalized by the total energy flux. In $(b)$, dashed lines MN and MS correspond to the locations of mooring $\mathrm{N}$ and $\mathrm{S}$ of the EXITS study, respectively. . . . . . . . . . . 49

2-18 Modal decomposition of the net energy flux of the generated internal tide before, after, and at locations MN and MS on the topography of the N-S transect shown in figure $2-12$ normalized by the total energy flux. The profiles are decomposed using the $(a)$ local depth and $(b)$ far-field depth. The net energy flux in the far-field before the topography $\left(F_{n}^{R}\right)$ and after the topography $\left(F_{n}^{T}\right)$ are also plotted, and should roughly agree with the modal decomposition of the net energy flux at the profiles taken before and after the topography, respectively. . . . . 50 
2-19 Normalized incident-plus-scattered horizontal velocity field $\left(u(x, z, t) / u_{0}\right)$ at an arbitrary instant in time for mode-1 (incident from the left) scattering from the U-N transect shown in figure 2 2-10] . . . . . . . . . . . 51

2-20 (a) Modal decomposition of reflected $\left(F_{n}^{R}\right)$ and the transmitted $\left(F_{n}^{T}\right)$ energy flux and $(b)$ the local depth-integrated, time-averaged, acrossridge energy flux of the internal tide scattered by the U-N transect shown in figure $2-10$, Both are normalized by the incident mode-1 energy flux. In (b), dashed lines MU and MN correspond to the locations of mooring $\mathrm{U}$ and $\mathrm{N}$ of the EXITS study, respectively. . . . . . . . . . 52

2-21 Modal decomposition of the net energy flux of the incident-plus-scattered internal tide before, after, and at locations MU and MN on the topography of the U-N transect shown in figure $|2-11|$ normalized by the incident energy flux. The profiles are decomposed using the $(a)$ local depth and (b) far-field depth. The energy flux before the topography $\left(F_{n}^{R+i}\right)$ and after the topography $\left(F_{n}^{T}\right)$ are also plotted, and should roughly agree with the modal decomposition of the net energy flux at the profiles taken before and after the topography, respectively. . . . . . . . . . . 52

2-22 Normalized incident-plus-scattered horizontal velocity field $\left(u(x, z, t) / u_{0}\right)$ at an arbitrary instant in time for mode-1 (incident from the left) scattering the N-S transect shown in figure $2-10$. . . . . . . . . . . . . . 54

2-23 (a) Modal decomposition of the reflected $\left(F_{n}^{R}\right)$ and transmitted $\left(F_{n}^{T}\right)$ energy flux and (b) the local depth-integrated, time-averaged, acrossridge energy flux of the internal tide scattered by the N-S transect \begin{tabular}{|c|}
\hline shown in figure $2-10$, Both are normalized by the incident mode-1 en- \\
\hline
\end{tabular} ergy flux. In $(b)$, dashed lines MN and MS correspond to the locations

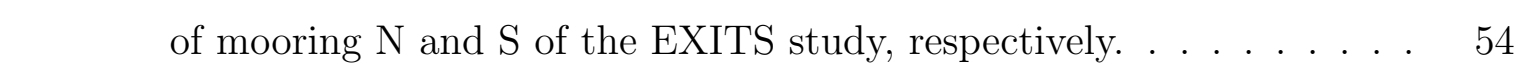


2-24 Modal decomposition of the net energy flux of the incident-plus-scattered internal tide before, after, and at locations MN and MS on the topography of the N-S transect shown in figure $|2-12|$ normalized by the incident energy flux. The profiles are decomposed using the $(a)$ local depth and (b) far-field depth. The energy flux before the topography $\left(F_{n}^{R+i}\right)$ and after the topography $\left(F_{n}^{T}\right)$ are also plotted, and should roughly agree with the modal decomposition of the net energy flux at the profiles taken before and after the topography, respectively. . . . . . . . . . . 55

2-25 (a) Energy flux of mode-1 $M_{2}$ internal tides around the Hawaiian ridge, estimated from multisatellite altimetry using a $120 \mathrm{~km}$ fitting region. The 4,000 $\mathrm{m}$ isobath contours are shown in gray. Red and green arrows indicate southward and northward internal tides, respectively. Flux arrows smaller than $0.15 \mathrm{~kW} / \mathrm{m}$ are not plotted. (b) Mode- $2 M_{2}$ internal tides. Flux arrows smaller than $0.05 \mathrm{~kW} / \mathrm{m}$ are not plotted. Adapted from Zhao et al. $[(2011)$.

3-1 Example of oceanographic data with image loss throughout the vertical domain. ........................ 67

3-2 (a) Characteristic oceanic stratification used to generate wave field in figure $3-3$ and corresponding vertical modes for the $(b)$ horizontal velocity, $\hat{u}(z)$, and $(c)$ isopycnal displacement, $\hat{\eta}(z)$ fields. . . . . . . . 70

3-3 An idealized horizontal velocity field in a characteristic oceanic stratification plotted as a function of the spatial coordinates $x$ and $z$, and time $t$. The wave field was constructed in MATLAB using the known modal strengths $\left|\mathcal{U}_{1: 10}\right|=\left[\begin{array}{lllllllll}10 & 9 & 8 & 7 & 6 & 5 & 4 & 3 & 2\end{array}\right]$ and phases $\varphi_{1: 10}^{u}=\left[\begin{array}{lll}1 & 2\end{array}\right]$ $345678910] \times \pi / 5 \mathrm{rad}$. . . . . . . . . . . . . . . 70 
3-4 Modal decomposition results for the $u$ velocity profile (top row) and the isopycnal displacement $\eta$ (bottom row). Plots $(a)$ and $(c)$ display the modal strengths while $(b)$ and $(d)$ display the phases. The known inputs (black dots) are compared to the projection method results (blue circles) and regression method results (green squares). . . . . . . . . 71

3-5 The percent error in the first five modes $m$ of the idealized wave field evaluated using the analytical procedure accounting for image loss (solid lines) and the regression method (dashed lines) as a function of image loss from the top of the vertical domain (top row), the bottom of the vertical domain (middle row), and the bottom of the vertical domain with combined image loss of $3 \%$ at the top and $2 \%$ in the middle (bottom row). Plots $(a),(c)$, and $(d)$ display the results for the $u$ velocity $(b),(d)$, and $(e)$ display the results for the $\eta$ displacement fields. 73

3-6 The (a) topography of the Line Islands Ridge transect investigated as \begin{tabular}{|c|}
\hline well as the northward (leftward) and southward (rightward) propagat- \\
\hline
\end{tabular} \begin{tabular}{|c|}
\hline ing horizontal velocity field $(u(x, z, t))$ at an arbitrary instant in time \\
\hline of the generated internal tide. In $(b)$, the modal amplitude of the first \\
\hline
\end{tabular} ten modes of the northward propagating internal tide as determine by

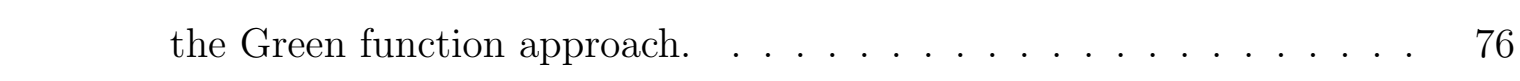

3-7 The modal amplitudes for the first five modes of the generated $M_{2}$ internal tide at the N-S transect of the Line Islands Ridge as determined by the Green function theory (blue line), projection method (red stars), and regression method (green circles). . . . . . . . . . . . . . 77

3-8 The percent error in computing the amplitudes of mode 1 (blue), mode 2 (green), and mode 3 (red) with varying $M$ when using the projection method (solid lines) and the regression method (dashed lines) for the idealized wave field presented in Section $\mid 3.4$ with 3\% top image loss and $60 \%$ bottom image loss. In $(a) M$ ranges 1 to 139 . Zoomed-in plots of the percent error for $M$ ranging from (b) 1 to 20 and (c) 60 to $139 . \ldots \ldots \ldots \ldots 79 \ldots \ldots \ldots$ 
3-9 The percent error in computing the amplitudes of mode 1 (blue), mode 2 (green), and mode 3 (red) with varying $M$ when using the projection method (solid lines) and the regression method (dashed lines) for the idealized wave field presented in Section $3.5 \mid$ with image loss consistent with mooring $U$ from the EXITS cruise. In (a) $M$ ranges 1 to 139. Zoomed-in plots of the percent error for $M$ ranging from $(b)$ 20 to 40 and $(c) 60$ to 139 . . . . . . . . . . . . . . 80

4-1 CTD/LADCP/microstructure stations were occupied from 12-36 hours and are labelled in order of occupation (dots). T011 and T015 are actually the same location but occupied at different times and slightly offset in this and following figures for clarity. Mooring U, N, and $\mathrm{S}$ locations are noted (stars). Colored contours indicate topography at 500-m intervals starting at $1500 \mathrm{~m}$. . . . . . . . . . . . . . 83

4-2 Instrumentation of moorings used in EXITS cruise and their average measurement locations at $(a)$ mooring $\mathrm{U},(b)$ mooring $\mathrm{N}$ and $(c)$ mooring S. Note that only instruments measuring velocity are shown. In the case of mooring $\mathrm{S},{ }^{*}$ indicates instruments only present during the first deployment and ${ }^{* *}$ indicates instruments only present during the second deployment. . . . . . . . . . . . . . . . . . . . 85

4-3 Samples of (left) raw measurements and (right) bandpassed semidiurnal signals at $(a),(b)$ mooring $\mathrm{U},(c),(d)$, mooring $\mathrm{N}$, and $(e),(f)$ mooring S. Isopycnal displacements with mean spacing of $100 \mathrm{~m}$ are plotted in black. . . . . . . . . . . . . . . . . . . . . . 87

4-4 Ocean stratification profiles at mooring N: $(a)$ buoyancy frequency profiles (in rad/s) from MMP measurements (green dashed line) and shipboard CTD data (blue line). (b) Normalized vertical structures of the first five baroclinic modes for pressure and horizontal velocity. (c) As in $(b)$, but for vertical displacement and vertical velocity. . . . . . . . 88 
4-5 Time series of the semidiurnal internal tide at mooring U. Semidiurnally bandpassed north-south velocity (color) along with isopycnal displacement (black lines) with mean spacing every $100 \mathrm{~m}$. . . . . . . 92

4-6 Time-varying modal amplitude for the $(a)$ north-south velocity and $(b)$ vertical displacement at mooring U for modes 1 (blue) and 2 (green). 93

4-7 $\quad$ Energy flux magnitude in modes 1 (blue), 2 (green), and 3 (red) for mooring U. . . . . . . . . . . . . . . . . . . . 93

4-8 Time series of the semidiurnal internal tide at mooring N. Semidiurnally bandpassed north-south velocity (color) along with isopycnal displacement (black lines) with mean spacing every $100 \mathrm{~m}$. . . . . . . 95

4-9 $\quad$ Time-varying modal amplitude for the $(a)$ north-south velocity and $(b)$ vertical displacement at mooring $\mathrm{N}$ for modes 1 (blue) and 2 (green). $\quad 96$

4-10 Energy flux magnitude in modes 1 (blue), 2 (green), and 3 (red) for mooring N. . . . . . . . . . . . . . . . . 96

4-11 Time series of the semidiurnal internal tide at mooring S. Semidiurnally bandpassed north-south velocity (color) along with isopycnal displacement (black lines) with mean spacing every $100 \mathrm{~m}$. . . . . . . . . . . 97

4-12 Time-varying modal amplitude for the $(a)$ north-south velocity and $(b)$ vertical displacement at mooring S for modes 1 (blue) and 2 (green). 98

4-13 Energy flux magnitude in modes 1 (blue), 2 (green), and 3 (red) for mooring S. . . . . . . . . . . . . . . . . . . . . . . . . . . . . . . . 99

4-14 Modal partition of $(a)$ energy and $(b)$ energy flux of the semidiurnal internal tide measured at moorings $\mathrm{U}, \mathrm{N}$, and $\mathrm{S}$. In $(a), \mathrm{HKE}$ is distinguished with a hatching pattern. . . . . . . . . . . . . . . . 100 
4-15 The $(a)$ total energy flux, $(b)$ mode-1 energy flux, and (c) mode-2 energy flux at the Line Islands Ridge as predicted by a high-resolution primitive equation model simulation. In $(a)$ every eight depth-integrated $M_{2}$ baroclinic energy flux vectors in each direction has been plotted. The underlying color gives the flux magnitude. Plots $(b)$ and $(c)$ zoom in on the EXITS study region. Contour interval is $1000 \mathrm{~m}$. Based on Carter et al. (2008) with data from conversations with Dr. Glenn Carter.103 


\section{List of Tables}

2.1 Mode-2 energy flux of the internal tide produced by generation and scattering of an incident mode-1 internal tide at the U-N and N-S transects. . . . . . . . . . . . . . . . 57

4.1 EXITS profiling moorings _................... . . . 84 


\section{Chapter 1}

\section{Introduction}

This thesis investigates the scattering of internal tides by deep-ocean topography. First, the internal tide scattering process is examined through analytical methods. The insights gained from these studies are then used to assist the interpretation of data from the first dedicated field observations of the internal tide scattering process, the EXperiment on Internal Tide Scattering (EXITS) cruise.

The basics of internal waves are described in Section 1.1 of this introduction. Section 1.2 presents the theoretical formulation of internal waves. Following on, Section 1.3 describes the method of analyzing internal wave fields via their modal content, a key task in determining how much scattering has occurred. Section 1.4 provides a survey of previous research on internal tide scattering. Finally, an overview of the thesis is presented in Section 1.5 .

\section{$1.1 \quad$ Background}

Internal waves are propagating perturbations of the density structure within a densitystratified fluid. They oscillate within, rather than on the surface of, a fluid medium. These oscillations are maintained by a balance between the forces of gravity and buoyancy and can exist at the interface of two fluids of different densities as well as in continuously stratified fluids. Due to spatial variations in temperature and salinity, both the atmosphere and ocean are examples of continuously density stratified fluids 
and therefore sustain internal waves. In the atmosphere, it has been found that internal waves are important vehicles for momentum transport and thus greatly influence general circulation models (Alexander et al., 2006, Hines, 1960). In the ocean, internal waves play an important role in dissipating barotropic tidal energy and driving a substantial amount of vertical mixing in the ocean (Garrett \& Kunze, 2007) and are thus crucial in understanding the global ocean energy budget (Munk \& Wunsch, 1998). This thesis focuses on the case of oceanic internal waves.

Previous studies suggest that radiated internal tide energy is one of the main sources of energy flux into the deep ocean (Wunsch \& Ferrari, 2004). Furthermore, a global study of tidal dissipation utilizing satellite altimetric data revealed that 25$30 \%(\sim 1 \mathrm{TW})$ of the total tidal dissipation $(\sim 3.7 \mathrm{TW})$ occurs in the deep ocean, near areas of rough topography, as shown in figure 1-1 (Egbert \& Ray, 2001). Additional investigations through analytical methods (Pétrélis et al., 2006, Echeverri \& Peacock, 2010), numerical modeling (Carter et al., 2008), laboratory experiments (Echeverri et al., 2009) and field observations (Rudnick et al., 2003) have resulted in a relatively

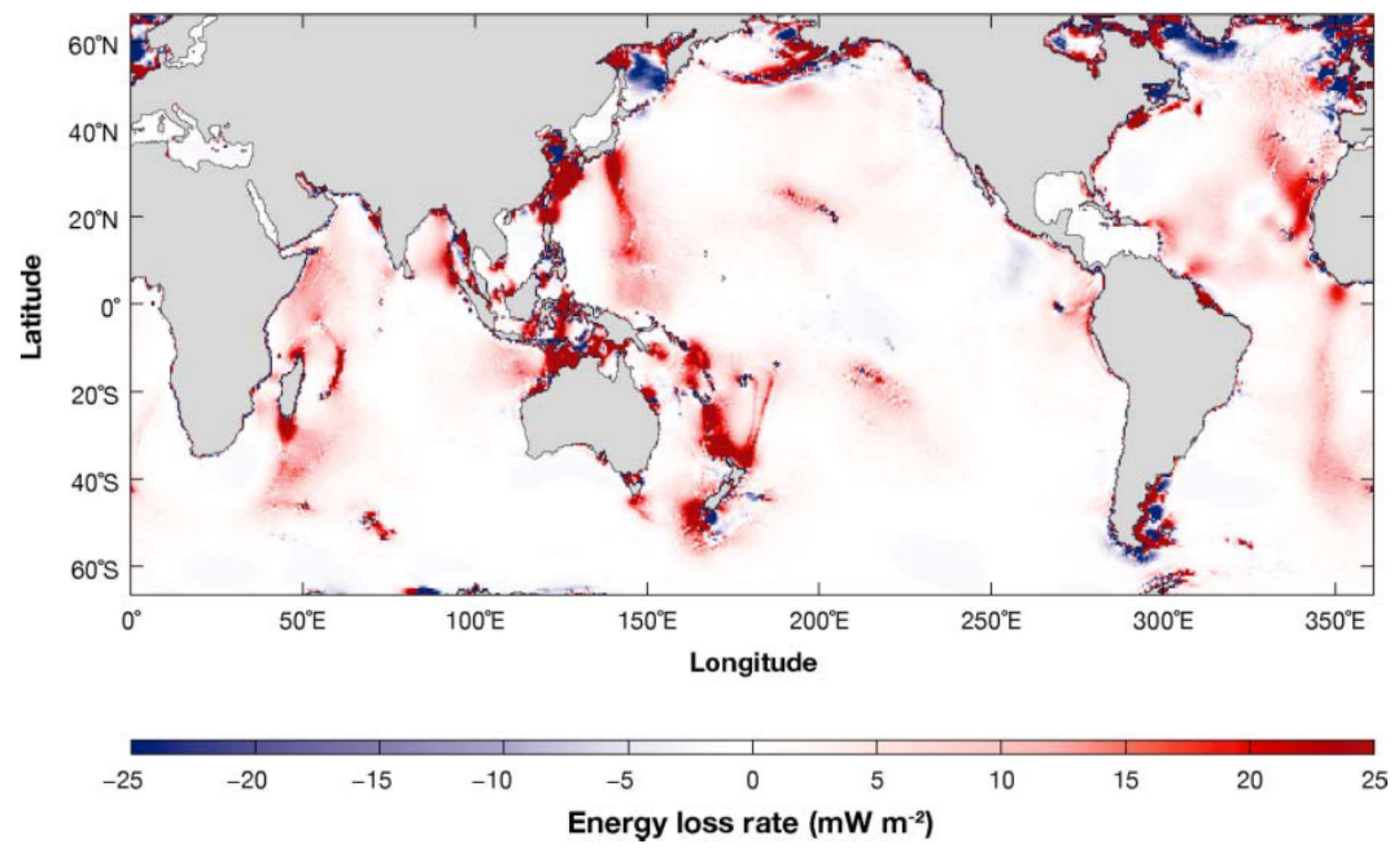

Figure 1-1: Energy loss from the barotropic tide calculated by Egbert and Ray (Egbert \& Ray, 2001) from satellite altimetry data. 
thorough understanding of the internal tide generation process in the ocean, although some outstanding issues remain regarding generation by highly three-dimensional topography.

Long internal waves can be decomposed into orthogonal normal modes or standing waves in the vertical, i.e. horizontally propagating and vertically standing waves whose spatial form is dictated by the vertical stratification (Gill, 1982). Extensive studies have found that the energy radiated by so-called high-mode internal tides (short-wavelength vertical modes, $n>2$ where $n$ is the mode number) dissipates near the generation site (Garrett \& Kunze, 2007). On the other hand, the majority of the energy radiated by significant topographic features propagates in the form of so-called low-mode internal tides (long-wavelength vertical modes, $n=1,2$ ). These low-mode internal tides have been observed via satellite altimetry data to travel far from the generation site, in some cases over a thousand kilometers away (Ray \& Mitchum, 1997, Zhao et al., 2012), and hence are more efficient at transporting energy than high-mode internal tides.

Current work focuses on determining where and by what means low-mode internal tide energy dissipates (Alford, 2003, MacKinnon \& Winters, 2005, Rainville \& Pinkel, 2006, Alford et al., 2007). Possible dissipation mechanisms include wave-wave interactions (Staquet \& Sommeria, 2002, MacKinnon \& Winters, 2005, Tsang et al., 2008), interactions with mean flows and mesoscale features (St. Laurent \& Garrett, 2002, Rainville \& Pinkel, 2006), topographic scattering in the deep ocean (Johnston \& Merrifield, 2003), and interaction with continental shelves (Kunze \& Llewellyn Smith, 2004). This thesis investigates the scenario of energy transfer of low-mode internal tides to higher modes via topographic scattering. 


\subsection{Governing equations}

Incompressible, adiabatic, and inviscid fluid motion in a rotating frame is governed by the momentum equation

$$
\frac{D \mathbf{u}}{D t}+2 \mathbf{\Omega} \times \mathbf{u}+\frac{\nabla p}{\rho}+\mathbf{g}=0
$$

mass conservation

$$
\frac{\partial \rho}{\partial t}+\nabla \cdot(\rho \mathbf{u})=0
$$

and incompressibility

$$
\nabla \cdot \mathbf{u}=0
$$

Here, a two-dimensional system is considered where $\mathbf{u}=(u(x, z, t), w(x, z, t))$ is the velocity field in the vertical plane $(x, z), \Omega$ is the Earth's rotation rate $(|\Omega|=7.29 \times$ $\left.10^{-5} \mathrm{rad} / \mathrm{s}\right)$ and is aligned with the vertical, $\rho(x, z, t)$ is the fluid density, $p(x, z, t)$

is the pressure, $\nu$ is the kinematic viscosity, and $\mathbf{g}=-g \hat{\mathbf{k}}=-9.81 \mathrm{~m} / \mathrm{s}^{2}$ is the gravitational acceleration.

Equations (1.1) through 1.3 can be linearized for small perturbations about a background state. To do this, $\mathbf{u}, \rho$, and $p$ can be defined in terms of a perturbation from the base state as

$$
\begin{aligned}
\mathbf{u}(x, z, t) & =\mathbf{u}_{b}(x, z, t)+\mathbf{u}^{\prime}(x, z, t), \\
\rho(x, z, t) & =\rho_{b}(z)+\rho^{\prime}(x, z, t), \\
p(x, z, t) & =p_{b}(z)+p^{\prime}(x, z, t),
\end{aligned}
$$

where the base state is indicated by the subscript $b$, the small perturbation variables are indicated by primes, and additional horizontal pressure gradients or perturbations have been neglected. For a barotropic tide in a stratified ocean, the background density is

$$
\rho_{b}(z)=\rho_{0}+\bar{\rho}(z)
$$


where $\rho_{0}$ is the reference density at $z_{0}$ and $\bar{\rho}(z)$ is the ambient equilibrium stratification.

Applying Newton's law to a fluid element perturbed from its basic state leads to the buoyancy frequency $N(z)$, the natural frequency of oscillation associated with the background stratification:

$$
N^{2}(z)=-\frac{g}{\rho_{b}(z)} \frac{d \bar{\rho}(z)}{d z}
$$

In the case for internal tides in the ocean, the Boussinesq approximation holds since the fluid density does not depart much from a mean reference value, that is $|\bar{\rho}(z)| \ll \rho_{0}$. Therefore, $\rho_{b}(z) \approx \rho_{0}$, and the buoyancy frequency reduces to

$$
N^{2}(z) \approx-\frac{g}{\rho_{0}} \frac{d \bar{\rho}(z)}{d z}
$$

Thus, in the Boussinesq limit, the total density field can then be written explicitly as

$$
\rho(x, z, t)=\rho_{0}+\frac{\rho_{0}}{g} \int_{z}^{z_{0}} N^{2}\left(z^{\prime}\right) d z^{\prime}+\rho^{\prime}(x, z, t) .
$$

The expression for total pressure then simplifies to

$$
p(x, z, t)=-g \int_{H}^{z} \rho_{b}\left(z^{\prime}\right) d z^{\prime}+p^{\prime}(x, z, t)
$$

where $H$ is the total depth of the fluid.

The equations governing the perturbed quantities are obtained by substituting (1.4), (1.9), (1.10) and (1.11) into the equations of motion, (1.1)-(1.3), and linearizing by neglecting quadratically small terms. The resulting linear equations governing the perturbations are

$$
\begin{aligned}
\frac{\partial \mathbf{u}^{\prime}}{\partial t}+2 \boldsymbol{\Omega} \times \mathbf{u}^{\prime}+\frac{\nabla p^{\prime}}{\rho_{0}}+g \frac{\rho^{\prime}}{\rho_{0}} \hat{k} & =0 \\
\frac{\partial \rho}{\partial t}+w^{\prime} \frac{d \bar{\rho}(z)}{d z} & =0
\end{aligned}
$$


and

$$
\nabla \cdot \mathbf{u}^{\prime}=0
$$

For internal tides, the $\beta$-plane approximation holds because the curvature of the Earth is small in the spatial domain of internal tides, therefore equations 1.12)-(1.14), which are exact in curvilinear coordinates, are approximately valid in Cartesian coordinates. Note that this approximation does not hold in the case of near-inertial waves. Furthermore, the Coriolis term in the momentum equation (1.12) can be simplified via the $f$-plane approximation, which builds upon the $\beta$-plane approximation (LeBlond \& Mysak, 1978). Therefore, the inviscid, linearized two-dimensional Boussinesq equations of motion in the $f$-plane that govern perturbations to the barotropic flow are:

$$
\begin{aligned}
\frac{\partial u}{\partial t}-f v+\frac{1}{\rho_{0}} \frac{\partial p}{\partial x} & =0 \\
\frac{\partial v}{\partial t}+f u & =0 \\
\frac{\partial w}{\partial t}+\frac{g}{\rho_{0}} \rho+\frac{1}{\rho_{0}} \frac{\partial p}{\partial z} & =0 \\
\frac{\partial \rho}{\partial t}+w \frac{d \bar{\rho}(z)}{d z} & =0
\end{aligned}
$$

and

$$
\frac{\partial u}{\partial x}+\frac{\partial w}{\partial z}=0,
$$

where $f=2 \Omega \sin \theta_{\text {lat }}$ is Coriolis frequency, $\theta_{\text {lat }}$ is the latitude of interest, the $y$ component of the velocity perturbation $v$, arises due to background rotation, and the primes have been dropped.

By defining a streamfunction $\psi$ such that $(u, w)=\left(-\psi_{z}, \psi_{x}\right)$, which immediately satisfies the continuity equation (1.19), equations (1.15)-(1.19) can be combined into a single equation governing the propagation of internal waves:

$$
\left(N^{2}(z)+\frac{\partial^{2}}{\partial t^{2}}\right) \psi_{x x}+\left(f^{2}+\frac{\partial^{2}}{\partial t^{2}}\right) \psi_{z z}=0
$$




\subsection{Internal wave modes}

An internal tide is a solution of the internal wave equation (1.20) with the same periodic time dependence as the forcing barotropic tide. Furthermore, since the spatial and temporal variables are independent, the time-dependence of the problem can be separated. Additionally, having assumed no viscous dissipation, the solution is periodic in both time and space. This results in seeking a separable solution of the form

$$
\psi(x, z, t)=\Re\left\{\phi(z) e^{i(k x-\omega t)}\right\},
$$

where $k$ is the wavenumber, $\phi(z)$ is the vertical structure, and $\omega$ is the frequency of the forcing barotropic tide. Substituting (1.21) into (1.20) yields a homogenous differential equation for $\phi(z)$,

$$
\frac{d^{2} \phi(z)}{d z^{2}}+k^{2}\left(\frac{N^{2}(z)-\omega^{2}}{\omega^{2}-f^{2}}\right) \phi(z)=0,
$$

which is subject to the boundary conditions of a flat bottom and rigid lid (since from the definition of the streamfunction, $\left.w=\psi_{x}=\Re\left\{i k \phi(z) e^{i(k x-\omega t)}\right\}\right)$ :

$$
\phi(z=0)=\phi(z=H)=0
$$

For a given buoyancy frequency profile $N(z)$, there exists a range of values for

$k$ for which an infinite number of nontrivial solutions to 1.22 exist. These specific values of $k$ are known as eigenvalues and their corresponding solutions $\phi(z)$, are known as eigenfunctions or more specifically, vertical modes. Furthermore, since (1.22) represents a Sturm-Louivile problem, the vertical modes form a basis and therefore any linear solution can be expressed as a superposition of these modes. In the case of a uniform stratification, $N(z)=N$ constant, 1.22 becomes a constant coefficient problem which admits sinusoidal normal modes given by

$$
\phi_{n}(z)=\Phi_{n} \sin \left(\frac{n \pi}{H}\right), \quad n=1,2,3, \ldots,
$$



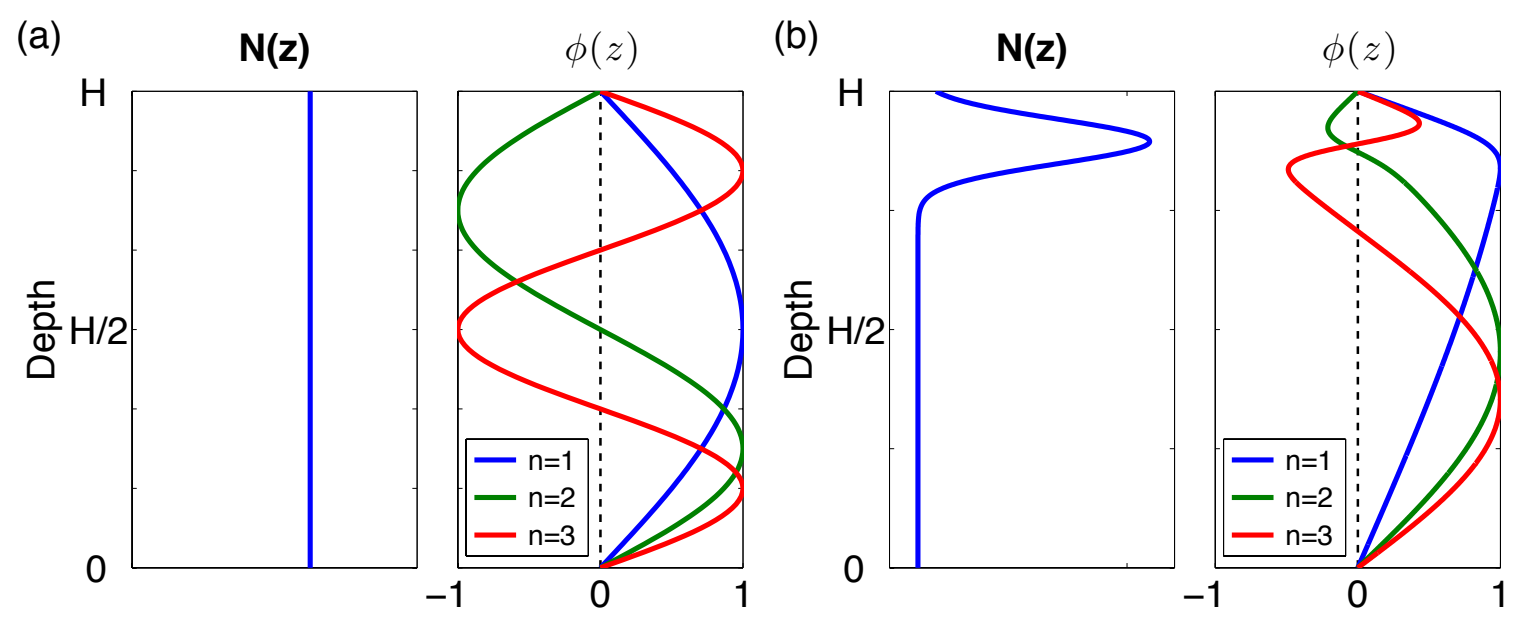

Figure 1-2: The first three modes, $\phi_{n}(z)$, for $(a)$ a uniform stratification profile, $N(z)=N$, and $(b)$ a nonuniform stratification profile, $N(z)$, characteristic of the ocean. In $(b)$, the vertical profiles indicate a concentration of amplitude and highest gradients in the vicinity of the peak of $N(z)$, the pycnocline.

where each value of the integer $n$ corresponds to a distinct vertical mode and $\Phi_{n}$ is the modal amplitude. An example of the first three mode shapes for a uniform stratification are shown in figure 1-2 (a). In the case of a nonuniform stratification, $N(z)$, no closed form solution exists to 1.22 except for a few specific $N(z)$ profiles. Therefore, numerical schemes must be used to solve for both the eigenvalues, $k_{n}$, and corresponding vertical modes, $\phi_{n}(z)$. Figure 1-2 $(b)$ depicts the first three mode shapes for a nonuniform stratification characteristic of the ocean.

To aid in comparison between wave fields, internal tides are described by their modal content. An internal tide dominated by low-modes means that the first few modes $(n=1,2)$ contain the majority of the energy flux of the internal tide. Similarly, an internal tide whose energy flux is mostly contained in modes other than the first few $(n=3,4,5, \ldots)$ is considered to be dominated by high-modes. This distinction is extremely important since the energy flux of an internal wave field scales as $\sim \frac{a_{n}^{2}}{n}$ (Echeverri et al., 2009), where $a_{n}$ is the modal amplitude and $n$ is the mode number. Thus, while an internal wave field may be comprised of two modes of the same amplitude, the lower mode is responsible for the majority of the energy flux. This thesis will also utilize this convention when comparing wave fields in Chapter 2. Furthermore, although this section presented the modes corresponding to the vertical 
velocity, $w$, it is clear that since the streamfunction is related to both the horizontal and vertical velocities by $(u, w)=\left(-\psi_{z}, \psi_{x}\right)$, there exist modes for the horizontal velocity as well, which are different in shape, but related, to the vertical modes presented in this section. Similarly, modes corresponding to pressure and density can also be found by making use of the governing equations of momentum and mass conservation. Building upon the work of Echeverri et al. (2009), Saidi (2011), the method used in determining the modal content of an internal wave field is described in detail in Section 3.2 ,

\subsection{Internal tide scattering}

Topographic scattering is the process by which the energy from an incident wave field is redistributed into higher modes (shorter vertical wavelengths) after encountering a topographic feature. It is an unresolved process in the energy flow from largescale inputs, such as surface tides and winds, to internal tides to micro-scale mixing (figure 1-3). It has been proposed that the generation of low-mode internal tides is the most important path from barotropic tidal energy to abyssal mixing at mid-

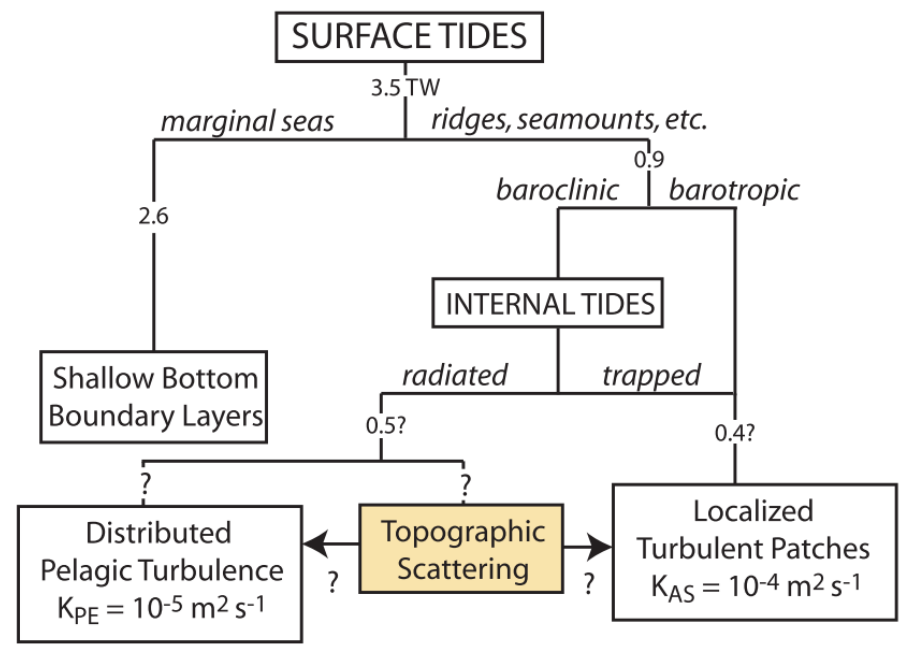

Figure 1-3: The global tidal energy budget depicting the energy transfers from the surface tides to mixing scales (modified from Munk \& Wunsch (1998)). More than $50 \%$ of the energy lost by the surface tide through boundary interactions is suggested to end up in internal waves that radiate away from the generation site. 
ocean ridges (St. Laurent \& Garrett, 2002). These low-mode internal tides are then hypothesized to scatter into higher modes as they propagate over rough topography, a conversion process suggested to be very efficient over rough ridges (Müller \& Xu, 1992, Polzin, 2004). These higher modes are more susceptible to energy dissipation via small-scale processes (such as shear instability and wave-wave interactions) resulting in internal-tide driven mixing at the scattering site.

The documentation of strong mixing at mid-ocean ridges (Polzin et al., 1997, Ledwell et al.) and the prevalence of rough topography in the ocean where low-mode internal tides are energetic (Niwa \& Hibiya, 2001), strongly suggest that topographic scattering is of global importance for understanding deep-ocean mixing and overturning circulation (Wunsch \& Ferrari, 2004). Theoretical studies of internal tide scattering suggest that the efficiency of scattering by finite-amplitude topography is more substantial than for infinitesimal topography (Müller \& Liu, 2000). This has been further implicated by satellite observations which suggest significant scattering of internal wave energy occurs at tall topography and only weak scattering over small-scale topography (Ray \& Cartwright, 2001, Ray \& Mitchum, 1997, Johnston et al., 2003, Zhao et al., 2012). In particular, Johnston et al. (2003) found that the altimetric sea surface height modulations due to the mode- $1 M_{2}$ internal tide propagating southward from the Hawaiian Ridge weaken at a prominent seamount in the Line Islands Ridge, where modulations due to the mode- $2 M_{2}$ internal tide increase, shown in figure 1-4, a clear indication of internal tide scattering.

Theoretical work has identified the criticality $\epsilon$ (i.e., ratio of the topographic slope to the internal ray slope) and height ratio $h^{*}$ (i.e., the ratio of the topographic height relative to the total water depth) to be the key parameters that determine the scattering efficiency of a two-dimensional topography. Specifically, scattering from low to high modes occurs most efficiently for near- and supercritical slopes (topographic slopes equal to and greater than the internal ray slope), and less so for subcritical slopes (Müller \& Liu, 2000). Furthermore, for small height ratios $\left(0<h^{*}<0.2\right)$, the scattering efficiency increases monotonically with $h^{*}$ and for larger height ratios $\left(0.2<h^{*}<1.0\right)$, scattering is most efficient near criticality (Mathur et al., 2014). 


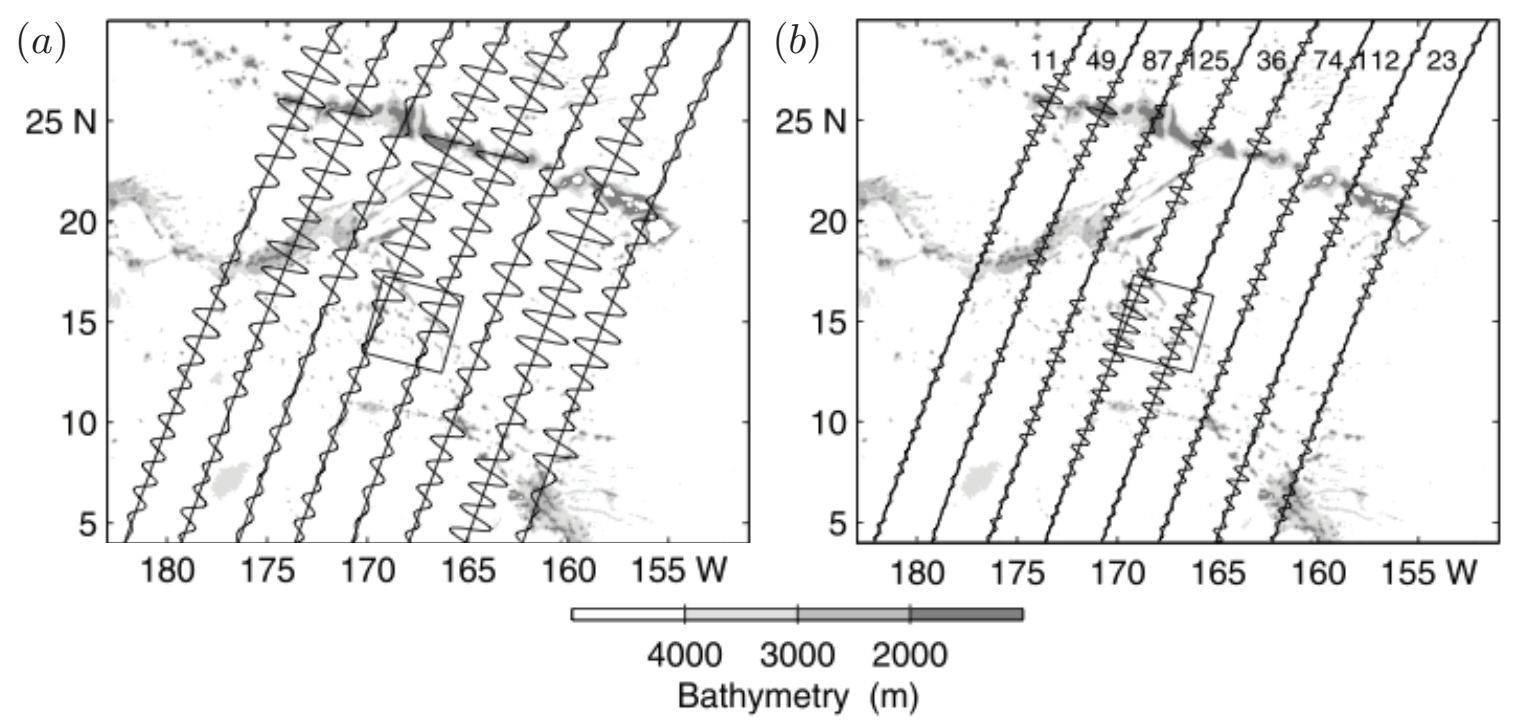

Figure 1-4: Altimeter sea surface height modulations due to the $(a)$ mode-1 and $(b)$ mode-2 $M_{2}$ internal tide. Grey shading shows bathymetry (Johnston et al., 2003)
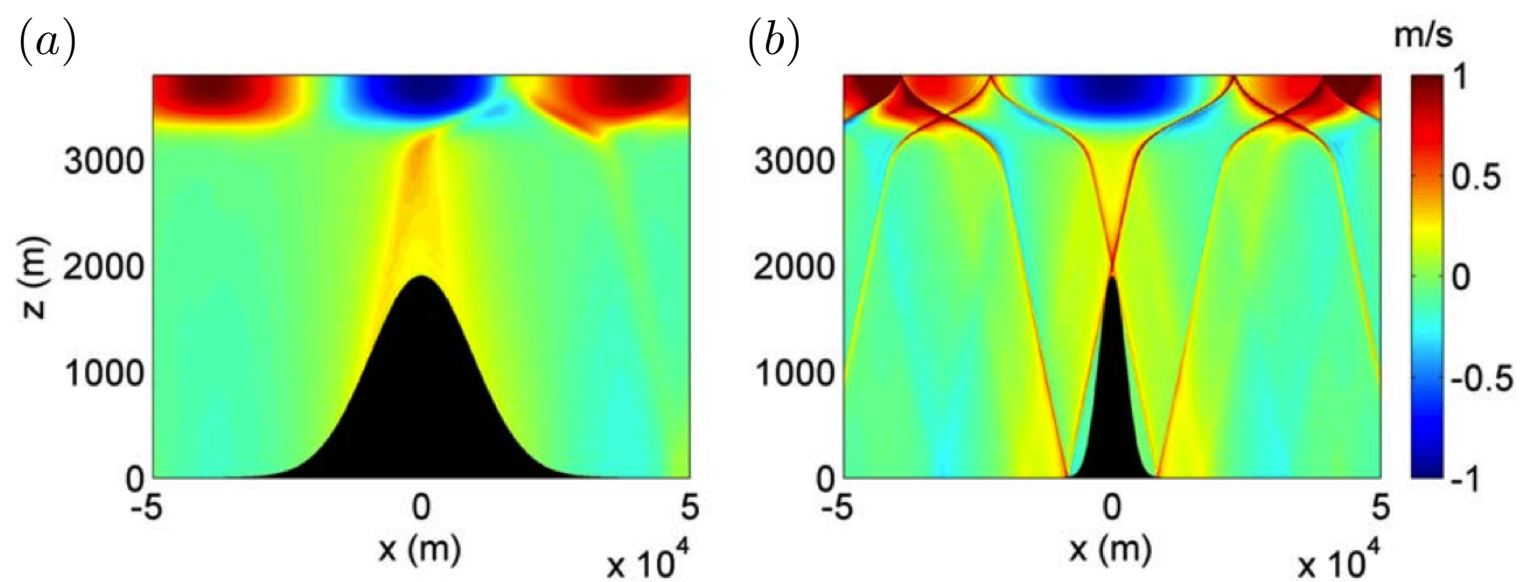

Figure 1-5: The horizontal velocity field $u(x, z, t)$ at an arbitrary instant in time for mode-1 internal tide (incident from the left) scattering in a nonuniform stratification by a (a) subcritical $(\epsilon=0.5)$ and (b) supercritical $(\epsilon=2)$ Gaussian ridge with $h^{*}=0.5$ (calculated using the Green function model) (Mathur et al. 2014 ).

Figure 1-5 depicts two scenarios from Mathur et al. (2014) in which the topographic feature $(a)$ does not (subcritical, $\epsilon=0.5)$ and $(b)$ does (supercritical, $\epsilon=2$ ) result in significant scattering of the incident mode-1 internal tide from a Gaussian ridge with $h^{*}=0.5$.

Detailed numerical studies of the scattering of a mode-1 internal tide by finiteamplitude Gaussian topography conclude that in general, the height of the topography 
affects the extent of energy transmission and reflection, while the slope and the width of the topography determine how much energy is scattered into higher modes (Johnston \& Merrifield, 2003). In particular, subcritical seamounts and ridges scatter less incident mode-1 internal wave energy than near- and supercritical features. When applied to study the scattering of a mode-1 internal tide generated at the Hawaiian Ridge by the Line Islands Ridge, it was found that around $19 \%$ of the incident energy scattered into modes 2-5 (Johnston et al., 2003$)$. This finding was part of the motivation for the first field observation of internal tide scattering, the EXITS cruise, conducted at the Line Islands Ridge (the results of which are presented in Chapter 4).

Further numerical studies of finite-amplitude low-mode internal waves incident on isolated topography suggest that that internal tide scattering by three-dimensional topographies is more suited for two-dimensional modeling than internal tide generation at three-dimensional topographies (Legg, 2014). Motivated by these findings, the Green function method, a two-dimensional analytical method of internal tide generation and scattering, was rigorously validated against nonlinear numerical simulations and utilized to examine the scenario of topographic scattering and predicted that $\sim 40 \%$ of a mode- 1 internal tide coming from the Hawaiian Ridge is scattered at the Line Islands Ridge (Mathur et al., 2014). Following this work, Chapter 2 applies the Green function method to examine the internal tide scattering process at the transects of the Line Islands Ridge studied during the EXITS cruise.

\subsection{Thesis overview}

The goal of this thesis is to analyze the scattering of the low-mode internal tide at the Line Islands Ridge. The theoretical formulation of the Green function method is outlined and utilized in Chapter 2 to examine the internal tide generation and scattering process in two dimensions at specific transects of the Line Islands Ridge that were study sites of the EXITS cruise. Given that the modal content of an internal wave field is the primary indicator of scattering, Chapter 3 describes and compares analytical and numerical techniques of wave field modal decomposition, particularly 
in cases of incomplete vertical domain information. Chapter 4 utilizes the modal decomposition methods outlined in Chapter 3 to analyze the results of EXITS, the first field experiment to study the internal tide scattering process. Finally, the thesis concludes with key findings in Chapter 5 . 


\section{Chapter 2}

\section{Analytical Model}

\section{$2.1 \quad$ Introduction}

In this chapter, the Green function approach is described, a theoretical framework in the limit of linear, inviscid and Boussinesq approximations for modeling the twodimensional interaction of internal waves with topography. While there are other analytical methods for solving the problem of both internal tide generation and scattering, namely Kelly et al. (2013), the focus of this thesis will be the established Green function approach, which has been thoroughly validated against full numerical modeling, as described in Mathur et al. (2014). Section 2.2 details the theory behind the Green function approach, while Section 2.3 utilizes it to predict the generated and scattered internal tide wave fields at an idealized Gaussian ridge, respectively. The method is then applied in Section 2.4 to examine the internal tide at two transects of the Line Islands Ridge that were the focus of the EXITS study, with the goal of aiding in the analysis of the observational field data whose results are described in Chapter 4. Section 2.5 discusses the scattering and generation properties of the transects using physical values of the barotropic and internal tides characteristic of the Line Islands Ridge. Finally, Section 2.6 concludes the chapter with suggestions for future work in using the Green function approach to investigate the modal content of generated and scattered internal wave fields along various topographies. 


\subsection{Theory}

Following the approach described by Robinson (1969), Pétrélis et al. (2006), Balmforth \& Peacock (2009), Echeverri \& Peacock (2010), and Mathur et al. (2014), a streamfunction $\psi(x, z, t)$ is defined such that the internal tidal velocities are given by $(u, w)=\left(-\psi_{z}, \psi_{x}\right)$, where $u$ is the horizontal velocity, $w$ is the vertical velocity, $x$ and $z$ are the horizontal and vertical coordinates, respectively, and $t$ is time. The streamfunction of the total wave field is

$$
\psi(x, z, t)=\psi_{b}(x, z, t)+\psi^{\prime}(x, z, t),
$$

where $\psi_{b}$ is the background internal tide and $\psi^{\prime}$ is the perturbation streamfunction due to internal tide generation or scattering by topography. The streamfunction is expected to be periodic in time and space with the same periodic time dependence as the forcing barotropic tide. Thus, the solution is assumed to be of the form

$$
\begin{aligned}
& \psi_{b}(x, z, t)=U \Re\left\{\phi_{b}(x, z) e^{-i \omega t}\right\} \\
& \psi^{\prime}(x, z, t)=U \Re\left\{\phi^{\prime}(x, z) e^{-i \omega t}\right\},
\end{aligned}
$$

where $U$ is the magnitude of the far-field barotropic tide in the case of internal tide generation or the incident mode-1 internal tide in the case of internal tide scattering, $\omega$ is the forcing frequency of the barotropic tide, and $\phi_{b}$ and $\phi^{\prime}$ are complex amplitudes representing the spatial portion of the streamfunction. The total spatial portion of the streamfunction is then given by $\phi(x, z)=\phi_{b}(x, z)+\phi^{\prime}(x, z)$. For the following studies, forcing by the $M_{2}$ barotropic tide is considered, for which $\omega=1.4053 \times 10^{-4} \mathrm{rad} / \mathrm{s}$. Note that due to the Coriolis acceleration, there exists a velocity component $v$, along the second horizontal direction $y$, which is given by

$$
v=U \Re\left\{\frac{i f}{\omega}\left(\phi_{b, z}+\phi_{z}^{\prime}\right)\right\},
$$

where the Coriolis frequency $f=2 \Omega \sin \theta_{\text {lat }}, \Omega$ is the Earth's background rotation 


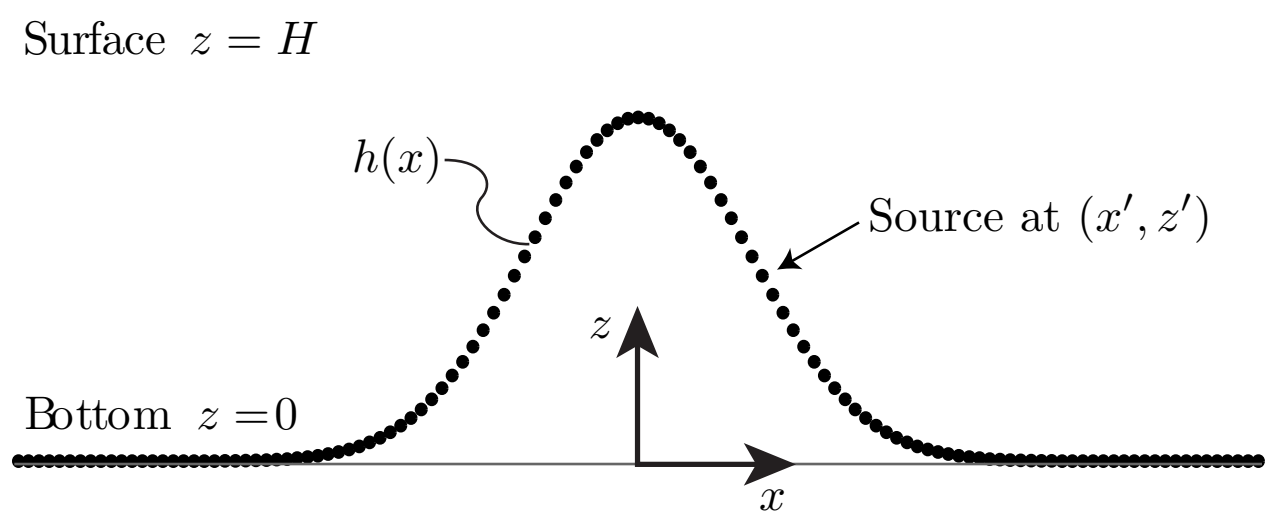

Figure 2-1: Problem geometry showing the boundaries and point-source distribution.

and $\theta_{\text {lat }}$ is the latitude of the region of interest.

Figure 2-1 depicts the problem geometry. In the Green function approach, the general solution for $\phi^{\prime}$ is expressed as an integral over contributions from a distribution of sources of strength $\gamma\left(x^{\prime}\right)$ placed on the topography $\left(x^{\prime}, z^{\prime}\right)=\left(x^{\prime}, h\left(x^{\prime}\right)\right)$ :

$$
\phi^{\prime}(x, z)=\int_{-a}^{b} \gamma\left(x^{\prime}\right) G\left(x, x^{\prime} ; z, h\left(x^{\prime}\right)\right) d x^{\prime}
$$

where $[a, b]$ are the horizontal limits of the topography and $G\left(x, x^{\prime} ; z, z^{\prime}\right)$ is the Green function, which is given by:

$$
G\left(x, x^{\prime} ; z, z^{\prime}\right)=\sum_{n=1}^{\infty} \frac{\Phi_{n}\left(z^{\prime}\right)}{2 k_{n}}\left(\int_{0}^{H} \frac{N^{2}(z)-\omega^{2}}{\omega^{2}-f^{2}} \Phi_{n}^{2} d z\right)^{-1} e^{i k_{n}\left|x-x^{\prime}\right|} \Phi_{n}(z)
$$

following standard procedures from Robinson (1969), Pétrélis et al. (2006), Echeverri $\&$ Peacock (2010), and Mathur et al. (2014). Here, $\Phi_{n}$ is the $n^{\text {th }}$ vertical mode satisfying the governing internal wave equation 1.22

$$
\frac{d^{2} \Phi_{n}}{d z^{2}}+k_{n}^{2}\left(\frac{N^{2}(z)-\omega^{2}}{\omega^{2}-f^{2}}\right) \Phi_{n}=0
$$

subject to the boundary conditions of no normal-flow through the ocean floor, $\Phi_{n}(z=$ $0)=0$ and the surface, $\Phi_{n}(z=H)=0$, approximated as a rigid lid. In (2.7), $k_{n}$ is the positive eigenvalue corresponding to the horizontal wavenumber and $H$ is the far-field 
ocean depth. The continuous function describing the topography $h(x)$ is assumed to be in the range $0 \leq h(x) \leq H$ for $x \in[-a, b]$, go smoothly to zero at $x=-a$ and $x=b$, and uniformly zero elsewhere. As described in Section 1.3, equation (2.7), with the homogeneous boundary conditions, constitutes a Sturm-Liouville system that must be solved numerically to obtain $\Phi_{n}$ for an arbitrary stratification $N(z)$. Without loss of generality, $\Phi_{n}$ is defined such that $\left|d \Phi_{n} / d z\right|$ has a maximum value of unity for $z \in[0, H]$.

Combining 2.5 and 2.6 yields the following expression for the perturbation streamfunction

$$
\phi^{\prime}(x, z)=\sum_{n=1}^{\infty}\left(\int_{0}^{H} \frac{N^{2}(z)-\omega^{2}}{\omega^{2}-f^{2}} \Phi_{n}^{2} d z\right)^{-1} \frac{\Phi_{n}(z)}{2 k_{n}} \int_{-a}^{b} \gamma\left(x^{\prime}\right) \Phi_{n}\left(h\left(x^{\prime}\right)\right) e^{i k_{n}\left|x-x^{\prime}\right|} d x^{\prime} .
$$

Since $\phi^{\prime}(x, z)$ is built out of the Green function $G\left(x, x^{\prime} ; z, z^{\prime}\right)$, which is comprised of vertical modes $\Phi_{n}(z)$ satisfying the boundary conditions of no normal-flow through the ocean floor topography $h(x)$ and sea surface, $\phi^{\prime}(x, z)$ automatically satisfies the following conditions

$$
\phi^{\prime}(x, h(x))=-\phi_{b}(x, h(x))
$$

and

$$
\phi^{\prime}(x, H)=0
$$

where $\phi_{b}$ is assumed to satisfy the no normal-flow boundary condition at $z=0$ and $z=H$. For the case of internal tide generation by barotropic forcing, as studied extensively in Echeverri \& Peacock (2010), the functional form of $\phi_{b}$ is

$$
\phi_{b}(x, z)=-U_{b} z
$$

where $U_{b}$ is the barotropic forcing velocity. In the case of scattering, an incident mode-1 internal tide is taken to propagate from left to right, in which case 


$$
\phi_{b}(x, z)=-U_{1} \Phi_{1}(z) e^{i k_{1} x}
$$

where here $U_{1}$ is the velocity of the incident mode- 1 internal tide. In this thesis, both the processes of internal tide generation and internal tide scattering are investigated via the Green function approach in order to better understand the extent to which the mode- 2 and mode-3 internal tide downstream of Line Islands Ridge, present in velocity data gathered by the EXITS cruise, is due to generation or scattering of the internal tide by the topography.

For the case of generation, substitution of (2.11) into (2.9) combined with the Green function solution (2.8) yields the following integral equation:

$$
U_{b} h(x)=\sum_{n=1}^{\infty}\left(\int_{0}^{H} \frac{N^{2}(z)-\omega^{2}}{\omega^{2}-f^{2}} \Phi_{n}^{2} d z\right)^{-1} \frac{\Phi_{n}(h(x))}{2 k_{n}} \int_{-a}^{b} \gamma\left(x^{\prime}\right) \Phi_{n}\left(h\left(x^{\prime}\right)\right) e^{i k_{n}\left|x-x^{\prime}\right|} d x^{\prime} .
$$

A similar integral equation can be found for the case of scattering, in which 2.12 is subtistuted into (2.9) and combined with the Green function solution (2.8):

$U_{1} \Phi_{1}(h(x)) e^{i k_{1} x}=\sum_{n=1}^{\infty}\left(\int_{0}^{H} \frac{N^{2}(z)-\omega^{2}}{\omega^{2}-f^{2}} \Phi_{n}^{2} d z\right)^{-1} \frac{\Phi_{n}(h(x))}{2 k_{n}} \int_{-a}^{b} \gamma\left(x^{\prime}\right) \Phi_{n}\left(h\left(x^{\prime}\right)\right) e^{i k_{n}\left|x-x^{\prime}\right|} d x^{\prime}$.

In (2.13) and (2.14), the topography $h(x)$, barotropic tidal velocity $U_{b}$, mode-1 internal tidal velocity $U_{1}$, forcing frequency $\omega$, Coriolis parameter $f$, and buoyancy frequency $N^{2}(z)$, are known parameters of the problem and $\Phi_{n}$ is found by solving (2.7), leaving the only unknown variable to be $\gamma(x)$. Both (2.13) and (2.14) can be solved numerically for $\gamma(x)$ using the procedure detailed in Echeverri \& Peacock (2010).

In the far field, where the ocean depth is constant, the quantities $\alpha_{n}^{ \pm}$can be defined as follows: 


$$
\alpha_{n}^{ \pm}=\frac{1}{2 k_{n}}\left(\int_{0}^{H} \frac{N^{2}(z)-\omega^{2}}{\omega^{2}-f^{2}} \Phi_{n}^{2} d z\right)^{-1} \int_{-a}^{b} \gamma\left(x^{\prime}\right) \Phi_{n}\left(h\left(x^{\prime}\right)\right) e^{\mp i k_{n} x^{\prime}} d x^{\prime}
$$

where $\alpha_{n}^{+}$corresponds to $x>b$ and $\alpha_{n}^{-}$corresponds to $x<-a$. Utilizing this definition of $\alpha_{n}^{ \pm}$, the perturbation stream function can be written as a sum over the vertical modes, where it is clear that $\alpha_{n}^{ \pm}$is the amplitude associated with the $n^{\text {th }}$ vertical mode:

$$
\phi^{\prime}(x, z)=\sum_{n=1}^{\infty} \alpha_{n}^{ \pm} \Phi_{n}(z) e^{ \pm k_{n} x}
$$

The total internal wave field in the case of the generation problem is given by $(2.11)$ and 2.16 as

$$
\phi(x, z)=-U_{b} z+\sum_{n=1}^{\infty} \alpha_{n}^{ \pm} \Phi_{n}(z) e^{ \pm k_{n} x}
$$

whereas for the scattering of an internal mode-1, it includes both 2.12 and 2.16 as

$$
\phi(x, z)=-U_{1} \Phi_{1}(z) e^{i k_{1} x}+\sum_{n=1}^{\infty} \alpha_{n}^{ \pm} \Phi_{n}(z) e^{ \pm k_{n} x}
$$

Of interest in studying both the scattering and generation of internal tides by barotropic velocities is the depth-integrated energy flux both transmitted $(T)$ and reflected $(R)$ by the topography. This value indicates the rate at which the baroclinic energy radiates away from the topographic region to the right or to the left, respectively. It is found by integrating the time-averaged far-field energy flux over the depth of the fluid. The instantaneous two-dimensional energy flux per unit of length along the topography, is given by

$$
\mathbf{J}=p \mathbf{u}
$$

The time-averaged far-field energy flux over the vertical domain is then 


$$
\begin{aligned}
F & =\int_{0}^{H}\langle\mathbf{J} \cdot \hat{\mathbf{1}}\rangle d z \\
& =-\int_{0}^{H}\left\langle p \psi_{z}\right\rangle d z \\
& =\int_{0}^{H}\langle p u\rangle d z
\end{aligned}
$$

where $p$ is the baroclinic pressure and the brackets indicate the time-average over a period of forcing. Utilizing (2.15), $F^{T, R}$ can be expressed as

$$
F^{T, R}=U^{2} \rho \omega\left(1-\frac{f^{2}}{\omega^{2}}\right) \sum_{n=1}^{\infty} \frac{\left|a_{n}^{ \pm}\right|^{2} \int_{0}^{H}\left(\frac{d \Phi_{n}}{d z}\right)^{2} d z}{2 k_{n}}
$$

and the depth-integrated energy flux in mode- $n$ is simply

$$
F_{n}^{T, R}=U^{2} \frac{\rho \omega}{2 k_{n}}\left(1-\frac{f^{2}}{\omega^{2}}\right)\left|a_{n}^{ \pm}\right|^{2} \int_{0}^{H}\left(\frac{d \Phi_{n}}{d z}\right)^{2} d z
$$

where $U$ is the magnitude of the far-field barotropic tide in the case of internal tide generation or the incident mode-1 internal tide in the case of internal tide scattering.

For internal tide generation, the transmitted $(\mathrm{T})$ and reflected $(\mathrm{R})$ depth-integrated energy flux in mode- $n$ will refer to the rightward and leftward propagating internal waves, respectively. Each of these can be normalized by the total energy flux as

$$
C_{n}^{T, R}=\frac{\left|\alpha_{n}^{ \pm}\right|^{2}}{k_{n}} \frac{\int_{0}^{H} \frac{d \Phi_{n}^{2}}{d z} d z}{\sum_{m=1}^{\infty} \frac{\left|\alpha_{m}^{ \pm}\right|^{2}}{k_{m}} \int_{0}^{H}\left(\frac{d \Phi_{m}}{d z}\right)^{2} d z} .
$$

For the scattering scenario, with the exception of transmitted mode 1, the transmitted and reflected depth-integrated energy flux in mode- $n$ can normalized by the incident energy flux in mode 1 as

$$
C_{n}^{T, R}=\left|\alpha_{n}^{ \pm}\right|^{2} \frac{k_{1}}{k_{n}} \frac{\int_{0}^{H}\left(\frac{d \Phi_{n}}{d z}\right)^{2} d z}{\int_{0}^{H}\left(\frac{d \Phi_{1}}{d z}\right)^{2} d z} .
$$

In the case of the energy flux in the transmitted mode 1 , the contribution from $\phi_{b}$ must be included as 


$$
C_{1}^{T}=\left|-1+\alpha_{1}^{+}\right|^{2}
$$

For convenience, the cumulative normalized transmitted and reflected energy fluxes in mode- $p$ to mode- $q$ can be defined as

$$
C_{p-q}^{T+R}=\sum_{n=p}^{q}\left(C_{n}^{T}+C_{n}^{R}\right)
$$

In order to completely represent the solution, 2.13) and 2.14 require $n=\infty$; in practice, however, this is not feasible. Therefore, the sum in 2.13 and 2.14 is truncated at a finite value of $n$ above which the solution changes negligibly. Additionally, the integrals in (2.13) and (2.14) require sufficient discretization of the spatial coordinates $x$ and $z$ for numerical convergence of the solution. In this study, scattering of the internal tide was investigated first, for which the spatial resolutions were increased until the energy conservation equation $C_{1-n}^{T+R}=1$ was satisfied with less than $1 \%$ error. This same spatial resolution was then utilized to examine the generation of the internal tide at the same transect.

Additionally, for convenience in the evaluation of the energy flux along the topography, (2.20) is reformulated as follows. Equation 2.19 can be written as

$$
\mathbf{J}=\psi p_{z} \hat{\mathbf{1}}-\psi p_{x} \hat{\mathbf{k}}+\nabla \times(\psi p \hat{\mathbf{j}})
$$

Applying the same operation that acts on $\mathbf{J}$ in 2.20 to 2.27 yields

$$
\begin{aligned}
F & =\int_{0}^{H}\langle\mathbf{J} \cdot \hat{\mathbf{i}}\rangle d z \\
& =\int_{0}^{H}\left\langle\psi p_{z}\right\rangle d z+\int_{0}^{H}\langle(\nabla \times(\psi p \hat{\mathbf{j}})) \cdot \hat{\mathbf{i}}\rangle d z
\end{aligned}
$$

Since $\partial / \partial y=$ for the two-dimensional problem, the second term in 2.28 can be reduced as follows: 


$$
\begin{aligned}
\int_{0}^{H}\langle(\nabla \times(\psi p \hat{\mathbf{j}})) \cdot \hat{\mathbf{1}}\rangle d z & =\int_{0}^{H}\left\langle\frac{\partial}{\partial y}(\psi p)\right\rangle d z \\
& =\left.(\psi p)\right|_{0} ^{H} \\
& =0
\end{aligned}
$$

by the boundary conditions of $\psi(x, 0, t)=\psi(x, H, t)=0$. Therefore

$$
F=\int_{0}^{H}\langle u p\rangle d z=\int_{0}^{H}\left\langle p_{z} \psi\right\rangle d z
$$

and for convenience it is the right-hand side of equation 2.30 that is evaluated. This has been implemented before by Llewellyn Smith \& Young 2002, 2003), Pétrélis et al. (2006) and Echeverri (2009), and the equivalence of either side of 2.30 has been confirmed by plotting the corresponding wave fields using the theory.

The analytical method described in this section has been incorporated by Mercier

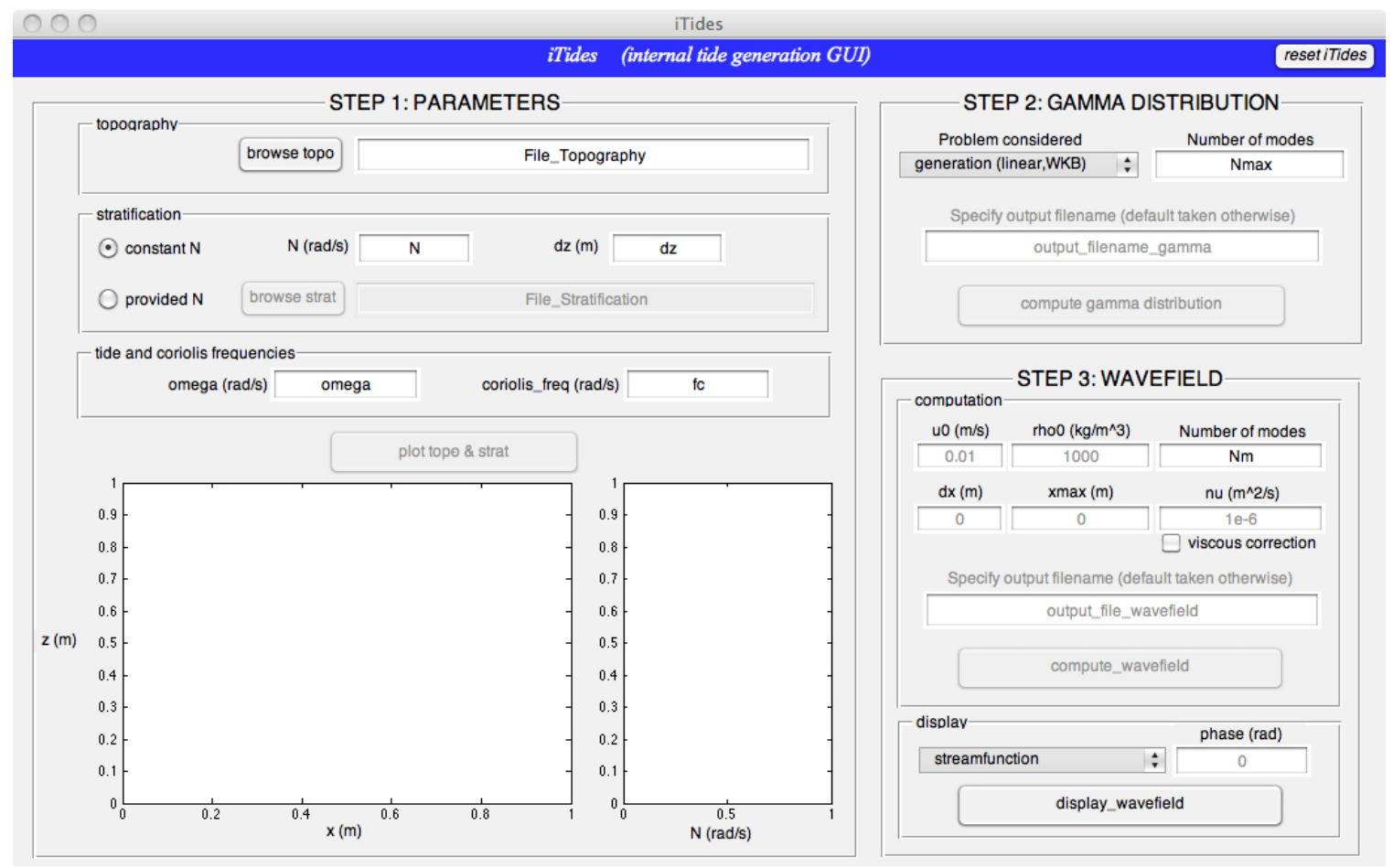

Figure 2-2: iTides Graphical User Interface (GUI). 
et al. (in prep.) into a new MATLAb toolkit known as iTides. Figure 2-2 presents a screenshot of the iTides graphical user interface. iTides was utilized in this thesis to carry out the Green function approach to predict the generation and scattering of oceanic internal tides by the two-dimensional topographies described in Sections 2.3 and 2.4. The GUI yields wave fields associated with the in-plane horizontal velocity $u$, out-of-plane horizontal velocity $v$, vertical velocity $w$, pressure $p$, vertical pressure gradient $p_{z}$, streamfunction $\psi$, and density perturbation $\rho$. The full details of $i$ Tides can be found in Mercier et al. (in prep.) and the toolbox itself can be downloaded from http://web.mit.edu/endlab/downloads/.

\subsection{Idealized Gaussian ridge}

The goal of this chapter is to better understand the generation and scattering aspects of the Line Islands Ridge, the focus of field experiments detailed in Chapter 4. To begin with, the generation and scattering of an idealized Gaussian ridge is examined to gain a basic understanding of how the modal composition of the internal tide varies far away from and over a topographic feature. The Green function approach detailed in Section 2.2 is used to predict both the generated wave field from a barotropic tide (Section 2.3.1) and the scattered wave field from an incident mode-1 internal tide (Section 2.3.2). Section 2.3.3 will further examine the relative generated and scattered internal tide at such a Gaussian ridge using physical values characteristic of the Line Islands Ridge. This section will also discuss what the strength of the forcing barotropic tide must be compared to an incident mode-1 internal tide for the generated and scattered energy flux in mode 2 to be of comparable order.

Figure 2-3 presents the topography of the idealized Gaussian ridge and the nonuniform stratification utilized by the Green function approach. The dimensions of the topography are characteristic of a transect of the Line Islands Ridge studied in the EXITS cruise and the nonuniform stratification is an idealized version of the oceanic stratification measured at the same transect. The latitude at this location, $17.1^{\circ} \mathrm{N}$, corresponds to $f=4.2898 \times 10^{-5} \mathrm{rad} / \mathrm{s}$. Additionally, the dashed lines labeled MN 
(a)

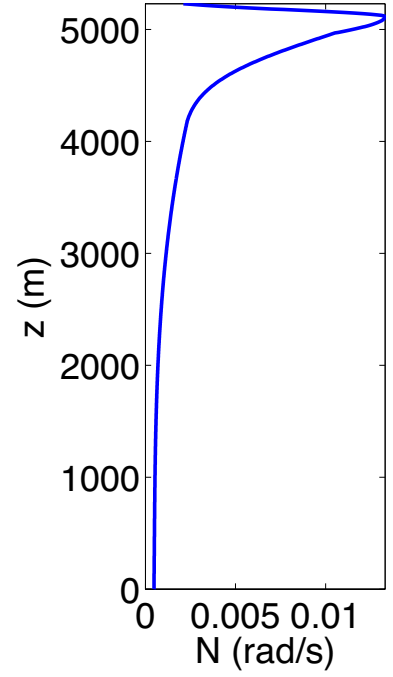

(b)

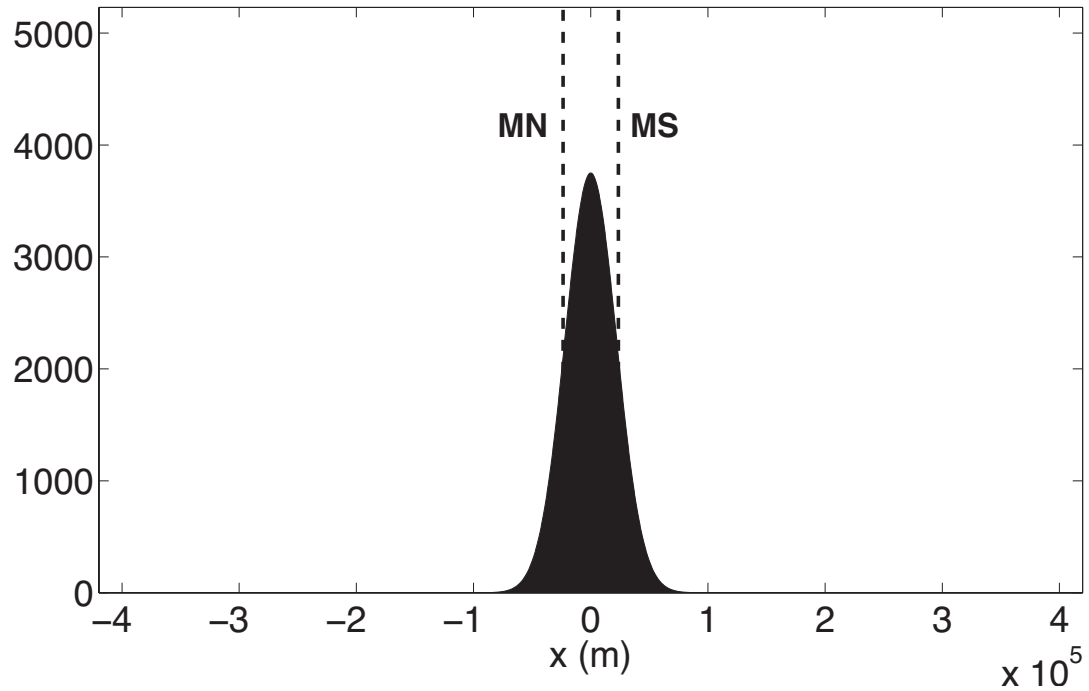

Figure 2-3: The $(a)$ nonuniform stratification and $(b)$ topography of the idealized Gaussian ridge representation of a characteristic transect of the Line Islands Ridge. In (b) the dashed lines $\mathrm{MN}$ and $\mathrm{MS}$ indicate effective locations of mooring $\mathrm{N}$ and $\mathrm{S}$ from the EXITS study, respectively.

and MS are situated on the topography at the effective locations of mooring $\mathrm{N}$ and $\mathrm{S}$ from the EXITS study, respectively.

\subsubsection{Generation results}

Figure 2-4 presents a snapshot of the theoretical results for the wave field generated by a barotropic tide at the Gaussian ridge as normalized by the tidal velocity, $u_{0}$. As can be seen from the figure, the wave field is symmetric, as expected, since both the barotropic tidal forcing and the topographic feature are symmetric. The presence of strong wave beams on either side of the topography indicate the presence of higher modes.

Figure 2-5( $a$ ) shows the modal decomposition of the leftward and rightward propagating internal tide normalized by the total energy flux. According to the Green function approach, the energy flux of the leftward and rightward propagating internal tide generated by the Gaussian ridge are identical, as expected for the symmetric topographic feature. Overall, mode-1 accounts for $40.3 \%$ of the energy flux of the generated internal tide and mode- 2 for $30.5 \%$. 


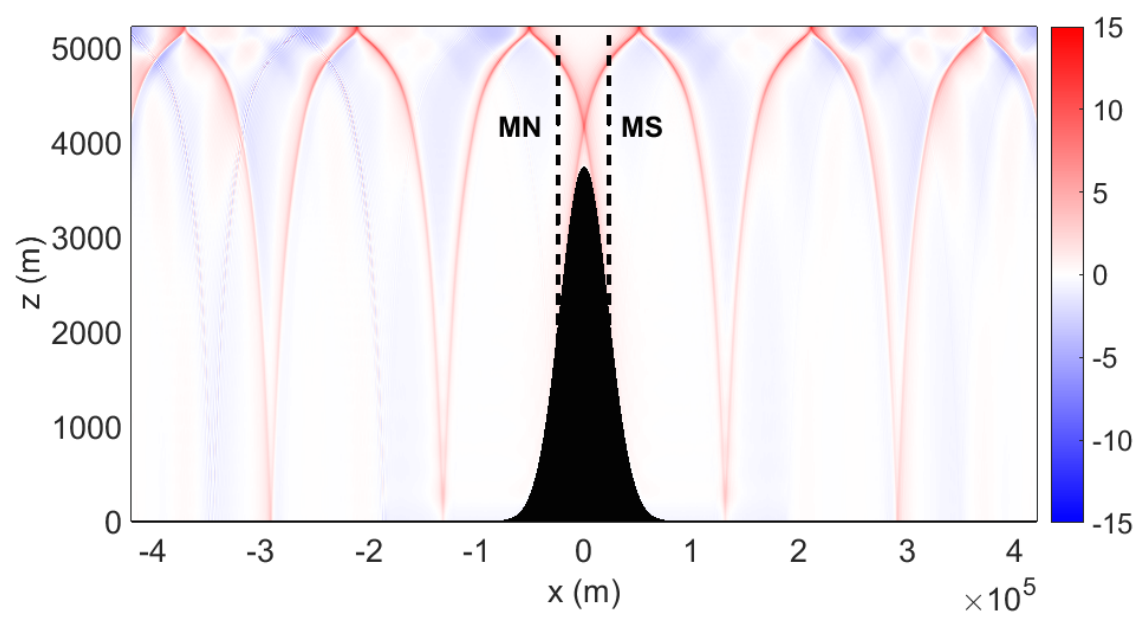

Figure 2-4: Normalized horizontal velocity field $\left(u(x, z, t) / u_{0}\right)$ at an arbitrary instant in time generated at the idealized Gaussian ridge depicted in figure 2-3.
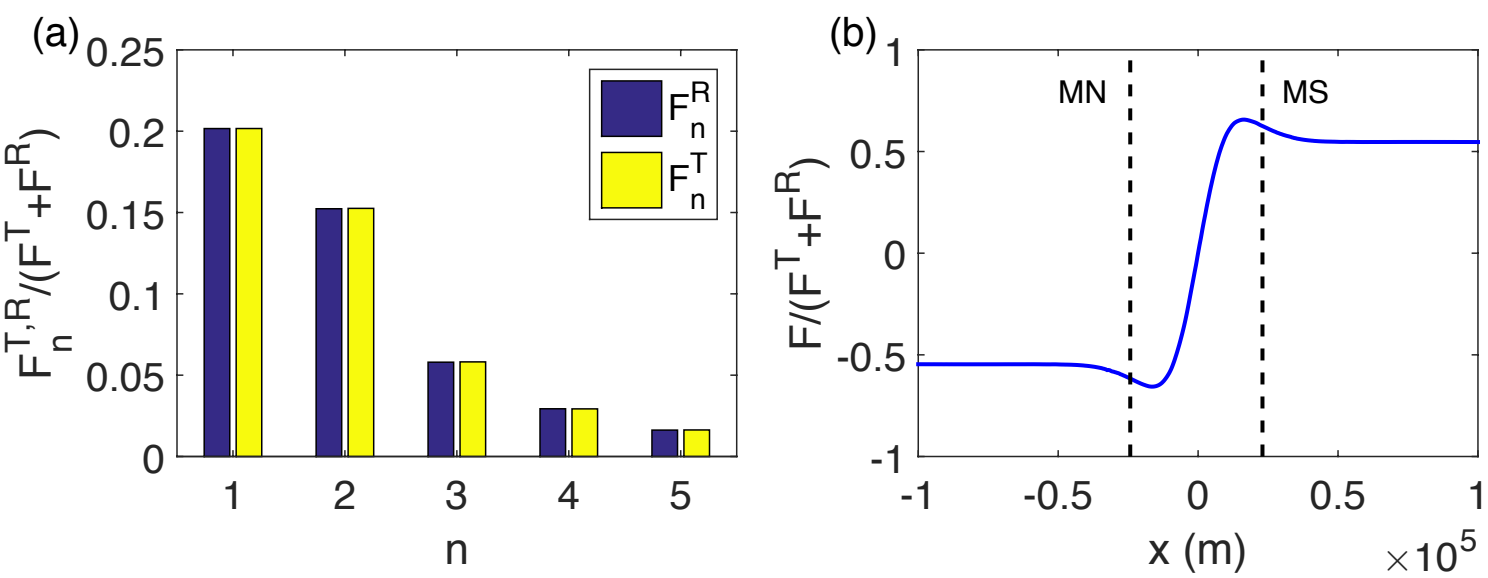

Figure 2-5: (a) Modal decomposition of the leftward $\left(F_{n}^{R}\right)$ and rightward $\left(F_{n}^{T}\right)$ propagating energy flux and $(b)$ the local depth-integrated, time-averaged, across-ridge energy flux of the generated internal wave field, both normalized by the total energy flux. In (b), dashed lines MN and MS correspond to the effective locations of mooring $\mathrm{N}$ and $\mathrm{S}$ of the EXITS study, respectively.

Figure 2-5(b) shows the local depth-integrated, time-averaged, across-ridge energy flux for the generated internal tide, as calculated by the right-hand side of 2.30). As seen in the figure, the energy flux at MN differs from that of the far-field leftward propagating internal tide by approximately $11.7 \%$, indicating that $\mathrm{MN}$ is not at a location of significant internal wave generation and therefore the modal content at this location should be similar to that of the far-field reflected internal tide. In the same way, the difference between the energy flux of far-field transmitted internal tide 
and that at MS is about $13.7 \%$, also indicating that the modal content at each of these locations should be similar. These results suggest that the internal wave fields at MN and MS are representative of their far-field counterparts.

The modal content of the generated internal tide can be further investigated. Harmonic velocity profiles at various locations in the wave field may be collected and modally decomposed using the technique outlined in Section 3.2. However, in this decomposition technique, an uncertain factor is the depth over which the flat bottom mode structure holds. Looking at figure 2-4, it is clear that the wave beam structure of the internal tide is unchanged at locations on the topography MN and MS, suggesting that the far-field ocean depth would yield accurate results. In using the far-field ocean depth, the presence of the topography results in image loss in the velocity profiles. However, in oceanographic field experiments, it is the local topographic depth at the locations of moored measurements that is used in the determination of the modal content of the wave field. The modal decomposition results when using both of these depth choices may be investigated and compared to the energy flux as predicted by equation 2.30 .

Figure 2-6 shows the results of modally decomposing the generated internal tide at locations before the topography, MN, MS, and after the topography. Note that the modal content at MN and MS are not expected to be identical because they are not on symmetric locations of the Gaussian ridge. Using the local topographic depth, figure 2-6(a), the sum of the energy flux in all modes at MN differs by over $40 \%$ with the energy flux computed at that location by 2.30 and shown in figure 2-5(b). Similarly, the total energy flux at MS is $45 \%$ less than the energy flux at MS in figure 2-5(b). On the other hand, using the far-field ocean depth, figure 2-6(b), the total energy flux at MN is within $2 \%$ of that shown in figure 2-5(b) at MN. The total energy flux at MS is also in excellent agreement, with less than 1\% difference between the energy flux at MS in figure 2-5(b). These results suggest that the far-field depth choice yields an accurate modal decomposition of the generated internal wave field.

Using the far-field depth modal decomposition, figure 2-6(b) shows that the modal decomposition of the generated internal tide before and after the topography match 
(a)

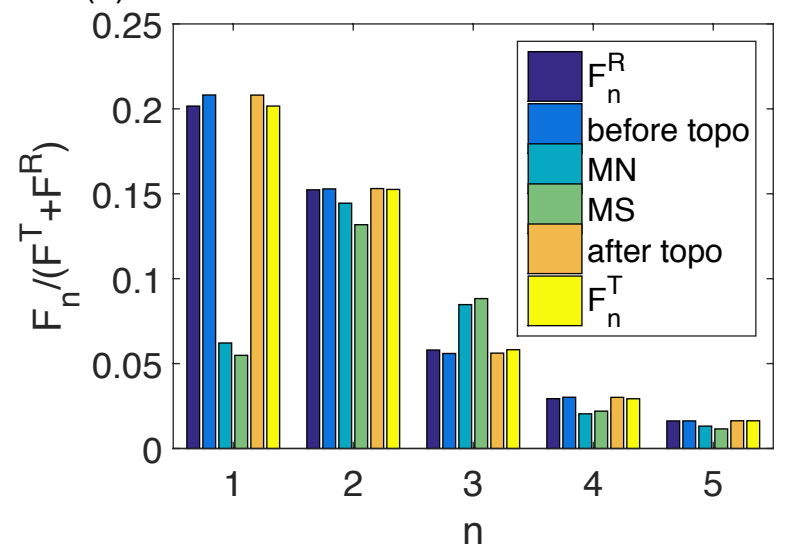

(b)

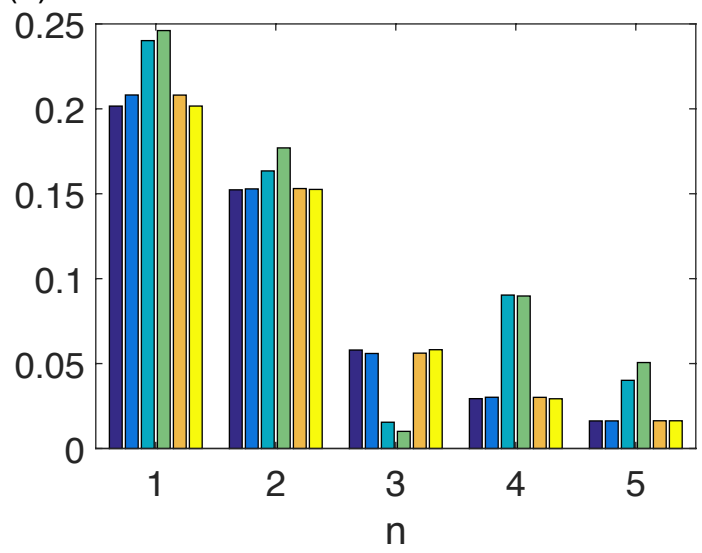

Figure 2-6: Modal decomposition of the net energy flux of the generated internal tide before, after, and at locations MN and MS on the topography of the Gaussian ridge shown in figure 2-3 normalized by the total energy flux. The profiles are decomposed using the $(a)$ local depth and (b) far-field depth. The net energy flux in the far-field before the topography $\left(F_{n}^{R}\right)$ and after the topography $\left(F_{n}^{T}\right)$ are also plotted, and should roughly agree with the modal decomposition of the net energy flux at the profiles taken before and after the topography, respectively.

very well with the modal content of the leftward and rightward propagating internal tides as determined by the Green function method. On the other hand, the modal decomposition of the internal tide at MN, although still dominated by modes 1 and 2 similar to the far-field leftward propagating internal tide, has content in modes 3-5 that differ greatly from its far-field counterpart. In particular, the energy flux at MN in mode 3 is $73.3 \%$ less, in mode 4 is $207.9 \%$ greater, and in mode 5 is $147.2 \%$ greater than the far-field leftward propagating internal tide. The modal content at MS also exhibits these differences with regard to the far-field rightward propagating internal tide. These results suggest the modal content of an internal tide at locations along the topography may not be a good indication of the modal content of the far-field propagating internal tides.

\subsubsection{Scattering results}

Figure 2-7 presents a snapshot of the theoretical results for the incident-plus-scattered wave fields resulting from a mode- 1 internal tide propagating from the left to right at the idealized Gaussian ridge. The horizontal velocity results presented have been 


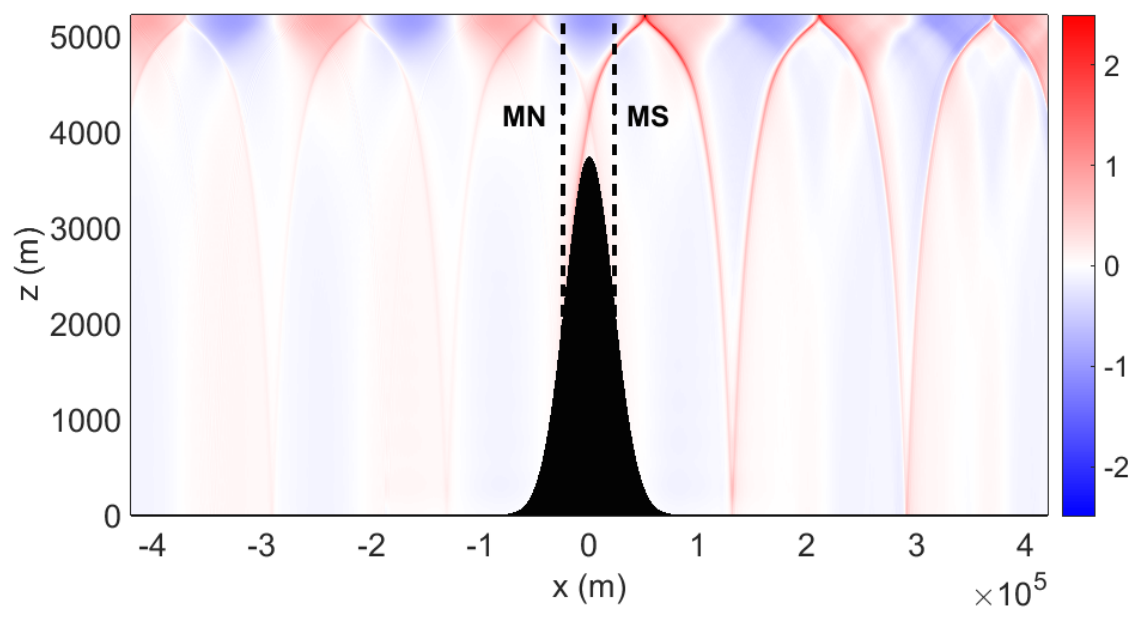

Figure 2-7: Normalized incident-plus-scattered horizontal velocity field $\left(u(x, z, t) / u_{0}\right)$ at an arbitrary instant in time for mode-1 (incident from the left) scattering from the idealized Gaussian ridge depicted in figure 2-3.

normalized by the velocity of the incoming mode- 1 internal tide, $u_{0}$. The presence of strong wave beams downstream of the topography clearly indicate scattering of the incident mode-1 internal tide. The weaker wave beams upstream indicate the presence of energy scattered into higher modes in the reflected wave field as well.

Figure 2-8(a) shows the modal decomposition of the transmitted and reflected energy flux normalized by the incident mode-1 energy flux. According to the Green
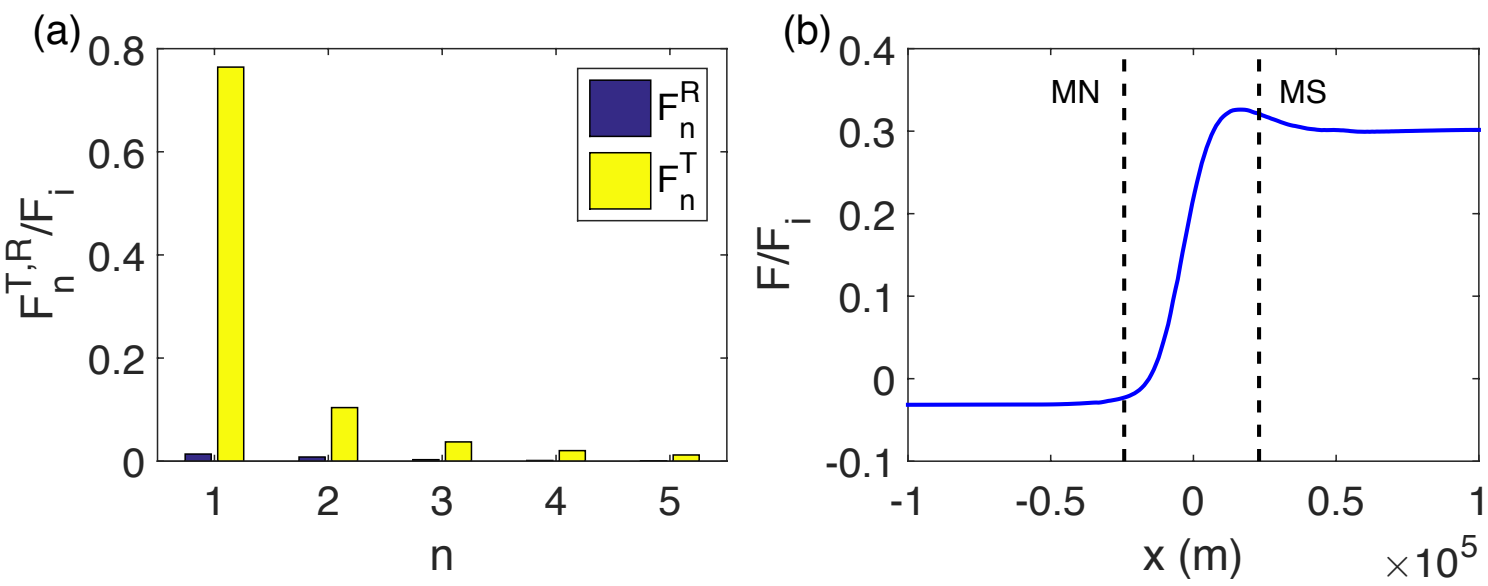

Figure 2-8: (a) Modal decomposition of the transmitted $\left(F_{n}^{T}\right)$ and reflected $\left(F_{n}^{R}\right)$ energy flux and $(b)$ the local depth-integrated, time-averaged, across-ridge energy flux of the scattered internal wave field, both normalized by the incident mode- 1 energy flux. In $(b)$, dashed lines MN and MS correspond to the locations of mooring $\mathrm{N}$ and $\mathrm{S}$ of the EXITS study, respectively. 
function approach, $97.2 \%$ of the incident mode-1 energy is transmitted and only $2.8 \%$ is reflected at the Gaussian ridge. Additionally, $22.2 \%$ of the incident mode- 1 energy is scattered into higher modes, either in the transmitted or reflected wave field, suggesting that a sufficient amount of scattering has taken place at the ridge. Most of the internal tide is scattered into mode- 2 , with an efficiency of $10.4 \%$ to the transmitted wave field and only $0.8 \%$ to the reflected wave field.

Furthermore, as shown in figure 2-8(b), the local depth-integrated, time-averaged, across-ridge energy flux, calculated by the right-hand side of 2.30 , of the scattered internal wave field changes considerably across the topography. At MN, the energy flux is fairly constant, nearly the same value as the far-field, indicating that a significant amount of scattering has not taken place at this location of the ridge. This suggests that the modal content of the wave field at MN should be approximately that of the northward far-field internal wave field. Similarly, the energy flux at MS is less than $5 \%$ greater than that of the far-field internal wave field to the south, also suggesting MS is not in a critical region of topographic scattering and that the modal content of the wave field at this location should approximate that of the far-field. This result suggests that for topographic features that are poor reflectors, the modal content of the internal tide is virtually unchanged until the peak of the topography, where scattering efficiently converts the energy flux into higher modes.

As done in Section 2.3.1, the modal content of the incident-plus-scattered internal tide field may also be investigated, the results of which are shown in figure 2-9. Motivated by the results from Section 2.3.1, it is expected that the modal decomposition technique when using the far-field ocean depth should yield correct results. However, unlike in the generation case, the modal content determined when using the local topographic depth, figure 2-9 (a) does not differ much from that determined when using the far-field depth, figure 2-9 (b). Furthermore, at MN, the distribution of modal content matches that of the energy flux before the topography, with the largest difference being $11.7 \%$ in the of mode 1 energy content when using the local topographic depth and $14.9 \%$ when using the far-field ocean depth. Similarly, at MS, the modal distribution of energy flux of the internal tide matches that of the far- 
(a)

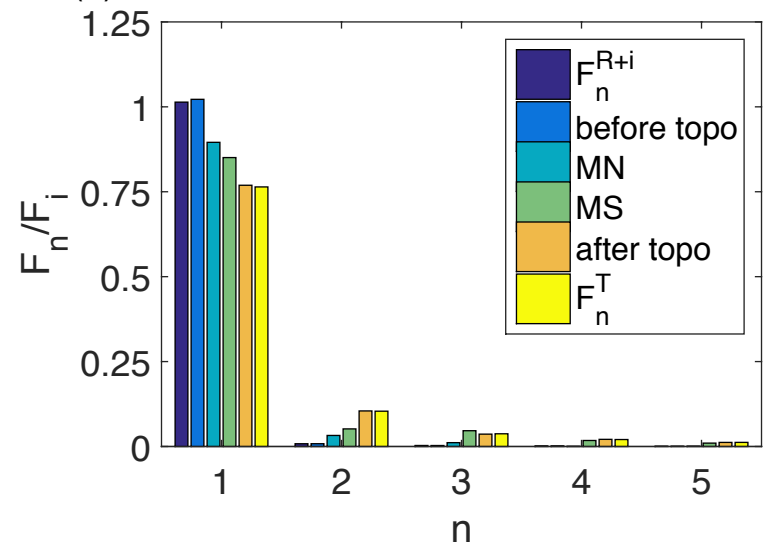

(b)

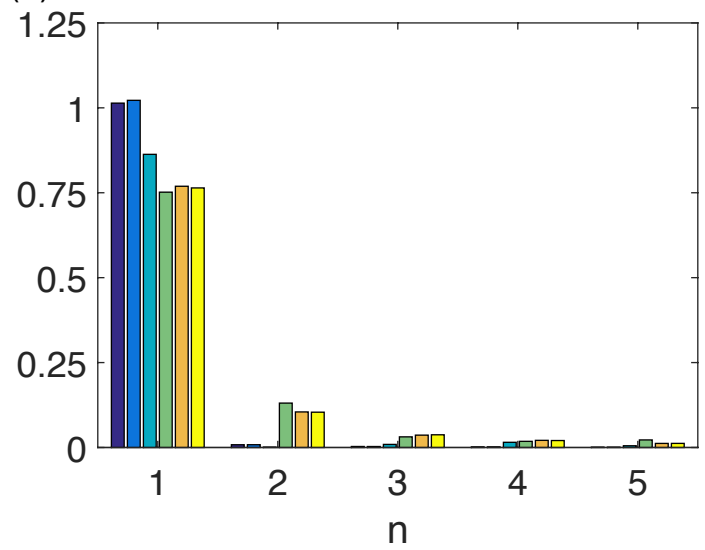

Figure 2-9: Modal decomposition of the net energy flux of the incident-plus-scattered internal tide before, after, and at locations MN and MS on the topography of the Gaussian ridge shown in figure $2-3$ normalized by the incident energy flux. The profiles are decomposed using the $(a)$ local depth and $(b)$ far-field depth. The energy flux before the topography $\left(F_{n}^{R+i}\right)$ and after the topography $\left(F_{n}^{T}\right)$ are also plotted, and should roughly agree with the modal decomposition of the net energy flux at the profiles taken before and after the topography, respectively.

field internal tide after the topography, only differing greatly in the modal content of mode- 2 by $50.1 \%$ when using the local topographic depth and $25.8 \%$ when using the far-field ocean depth. These results suggest that, unlike in the generation case, the modal decomposition depths used for a velocity field due to a scattered internal tide yield similar results and can thus be used interchangeably. Moreover, also in contrast to the generation case, the modal content at various locations along the topography are in good agreement with their far-field counterparts.

\subsubsection{Generation vs. Scattering}

One objective of this study is to quantify how much mode- 2 internal tide is due to scattering of incident mode-1 internal tide versus barotropic tidal generation at the Gaussian ridge. For this comparison, characteristic values of the incident mode-1 internal tide velocity, $u_{0}=0.05 \mathrm{~m} / \mathrm{s}$ (based on experimental measurements from the EXITS cruise and within the range of values used in numerical simulations by Johnston et al. (2003) and Ray \& Cartwright (2001)), and the barotropic tidal velocity, $u_{b}=0.03 \mathrm{~m} / \mathrm{s}$ (from TPXO tidal predictions at the Line Islands Ridge and within the 
range of values used in numerical studies by Echeverri \& Peacock (2010), Merrifield \& Holloway (2002) and Llewellyn Smith \& Young (2003) and reported in field studies by St. Laurent \& Nash (2004) and Lee et al. (2006)), were used to determine the dimensional energy flux in mode 2 due to the scattered and generated internal tide. With these values, approximately $0.14 \mathrm{~kW} / \mathrm{m}$ of the scattered energy flux is contained mode 2 as compared to $2.7 \mathrm{~kW} / \mathrm{m}$ in the generated mode- 2 internal tide. Therefore, although the ridge is efficient in scattering the incident mode- 1 internal tide into mode 2 , the mode-2 internal tide generated by the ridge is an order of magnitude greater than that due to the scattering of a incident mode-1 internal tide.

However, since the magnitude of $F$ scales with $U^{2}$ for both the generated and scattered internal tide by 2.22 , this introduces significant uncertainty into the predicted conversion rates, which can only be reasonably estimated within the bounds set by the uncertainty in $U$. For instance, with a barotropic tidal velocity of $u_{b}=0.01 \mathrm{~m} / \mathrm{s}$, the smallest value used in numerical and analytical studies of this region (Merrifield \& Holloway, 2002, Llewellyn Smith \& Young, 2002) the generated internal tide mode 2 energy flux is only $0.30 \mathrm{~kW} / \mathrm{m}$. In this case, an incident mode- 1 internal tide velocity of $u_{0}=0.074 \mathrm{~m} / \mathrm{s}$ or greater would yield a scattered wave field with $0.30 \mathrm{~kW} / \mathrm{m}$ or greater energy flux in mode 2 .

\subsection{Line Islands Ridge transects}

In preparation for rigorous analysis of data from the field study (presented in Chapter 4), this section seeks to analyze both the generation of internal tides by barotropic forcing and the scattering of a mode-1 internal tide at the Line Islands Ridge using the two-dimensional Green function method described in Section 2.2. The analytical study of the idealized Gaussian ridge in Section 2.3 demonstrated that a topography with a singular peak was efficient in both generating higher mode internal waves and scattering an incident mode-1 internal tide into higher mode internal waves. It also showed that although the majority of the scattered internal tide goes into mode 2 , the resulting wave field is still dominated by mode 1. Additionally, using characteristic 


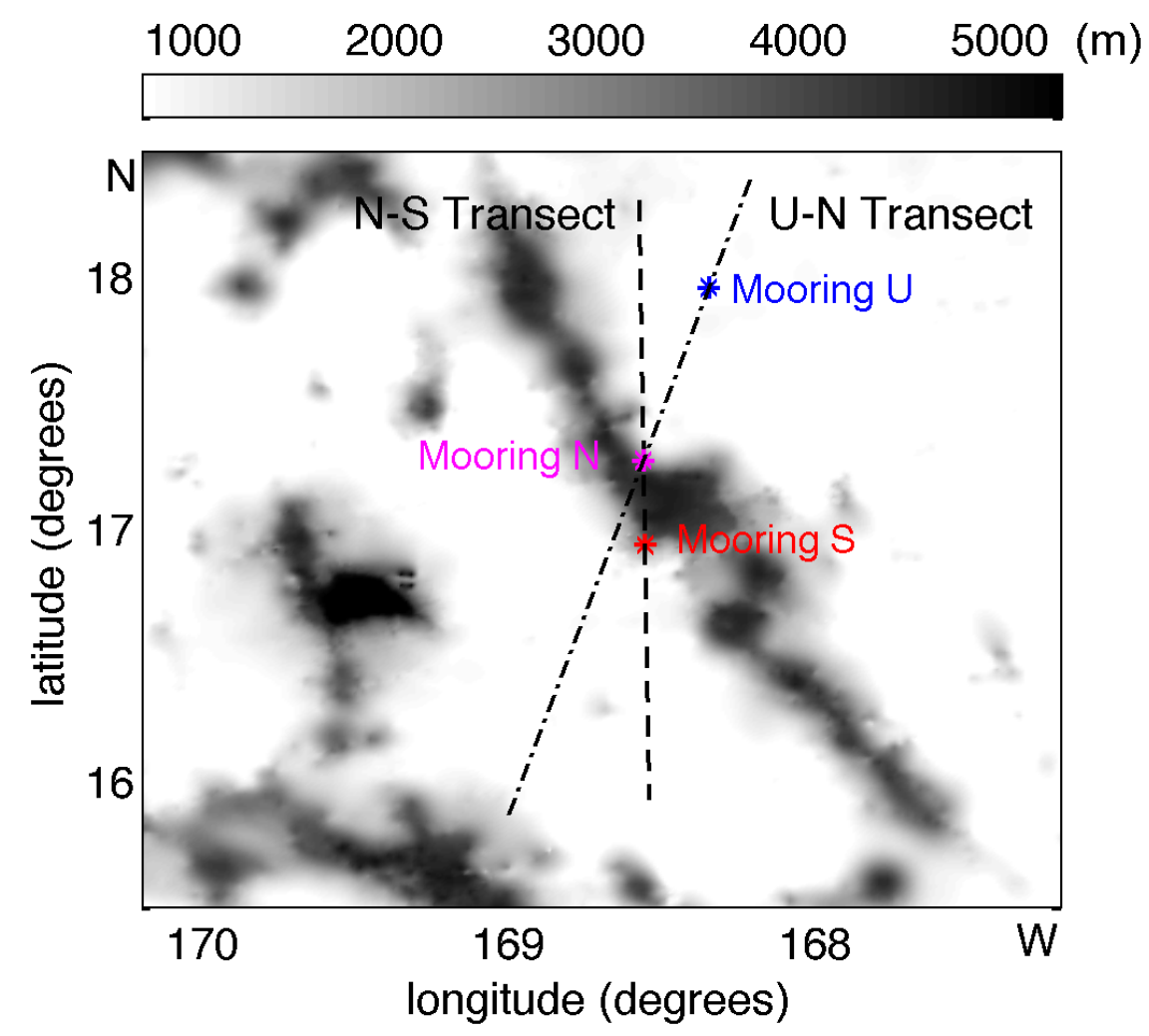

Figure 2-10: A contour plot of the topography of the Line Islands Ridge, indicating the locations of the three internal wave observation moorings from the EXITS cruise: mooring $\mathrm{U}$, mooring $\mathrm{N}$, and mooring $\mathrm{S}$. The scattering of a mode-1 internal tide and internal tide generation by barotropic forcing is investigated for transects between moorings $\mathrm{U}$ and $\mathrm{N}$ (U-N transect) and moorings $\mathrm{N}$ and $\mathrm{S}$ (N-S transect), indicated by the dashed lines.

values of the incident mode- 1 internal tide and barotropic forcing tide velocities, the mode-2 energy flux generated by the idealized ridge was shown to be much greater than that scattered. The expectation is these results will carry on to more realistic transects, as those from the Line Islands Ridge.

Figure 2-10 presents the contour plot of a roughly $3^{\circ}$ longitude by $3^{\circ}$ latitude section of the ridge system. In addition to the bathymetry, the figure also indicates the locations at which velocity data was gathered during the EXITS cruise (moorings $\mathrm{U}, \mathrm{N}$ and $\mathrm{S}$ ) and is analyzed in Chapter 4 for evidence of the topographic scattering of the internal tide. The generation and scattering of the internal tide at two crosssections in this domain between moorings $\mathrm{U}$ and $\mathrm{N}$ (U-N transect) and moorings $\mathrm{N}$ and S (N-S transect), indicated by the dashed lines in figure 2-10, are considered in 
(a)

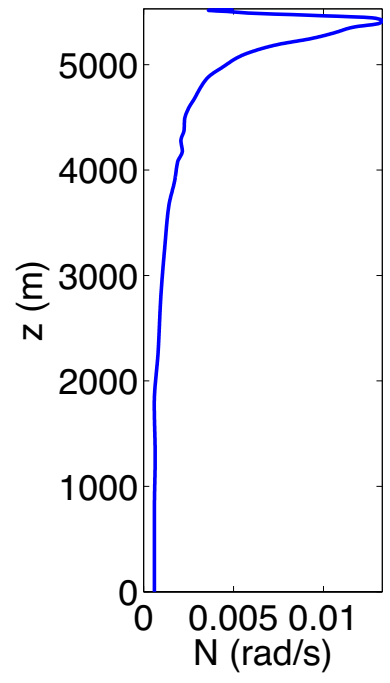

(b)

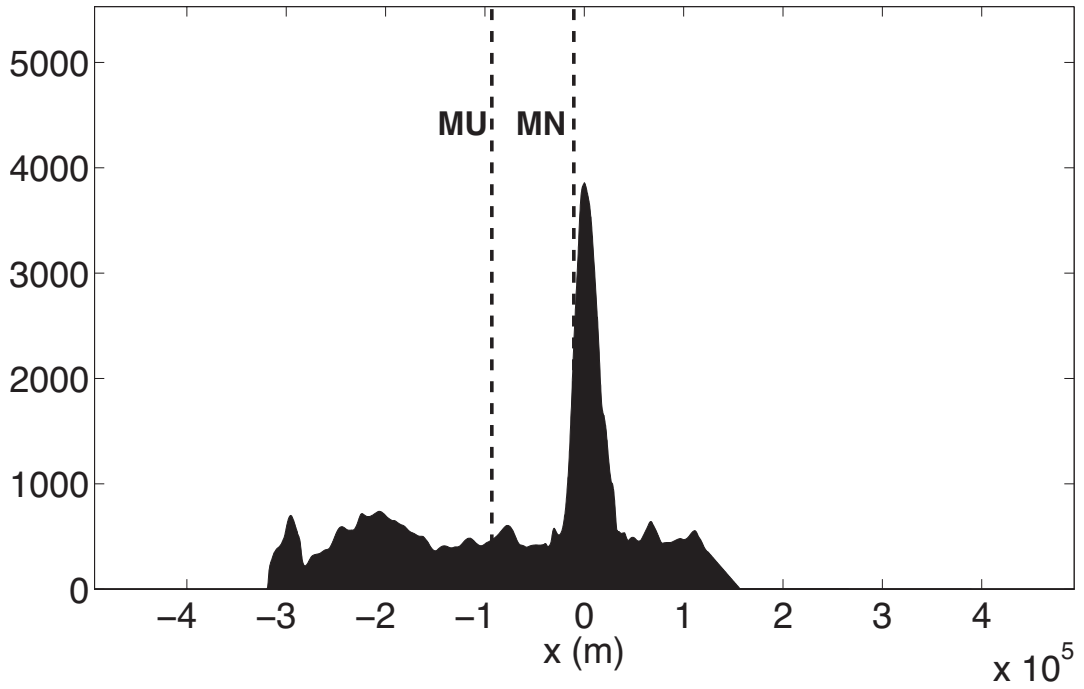

Figure 2-11: The $(a)$ stratification and $(b)$ topography of the U-N transect of the Line Islands Ridge shown in figure 2-10. In (b) the dashed lines MU and MN indicate effective locations of mooring $\mathrm{U}$ and $\mathrm{N}$ from the EXITS study, respectively.

this section.

Figure 2-11 depicts the topography and stratification at the U-N transect shown in figure 2-10, where the latitude, $17.6^{\circ} \mathrm{N}$, corresponds to $f=4.4135 \times 10^{-5} \mathrm{rad} / \mathrm{s}$. Additionally, figure 2-11(b) also includes the locations of mooring $\mathrm{U}$ and $\mathrm{N}$ from the EXITS study, MU and MN, respectively. As compared to the idealized Gaussian ridge from figure 2-3, the U-N transect has small scale topographic features leading up to the peak, which were not present in the Gaussian ridge. Moreover, while the peaks in both topographies are of similar height, the peak in the $\mathrm{U}-\mathrm{N}$ transect is much slimmer, more like a knife-edge than a Gaussian ridge. These topographic differences suggest that the generated and scattered internal tides will also vary between the topographies.

The topography and stratification at the N-S transect of figure 2-10 is depicted in figure 2-12, where the latitude, $17.1^{\circ} \mathrm{N}$, corresponds to $f=4.2898 \times 10^{-5} \mathrm{rad} / \mathrm{s}$. As in figure 2-11(b), the dashed lines in figure 2-12(b) indicate the locations of mooring $\mathrm{N}$ and $\mathrm{S}$ from the EXITS study, MN and MS, respectively. Unlike the U-N transect, the idealized Gaussian topography studied in Section 2.3 was based on the N-S transect and therefore the two topographies share many similar features. Both to- 
(a)

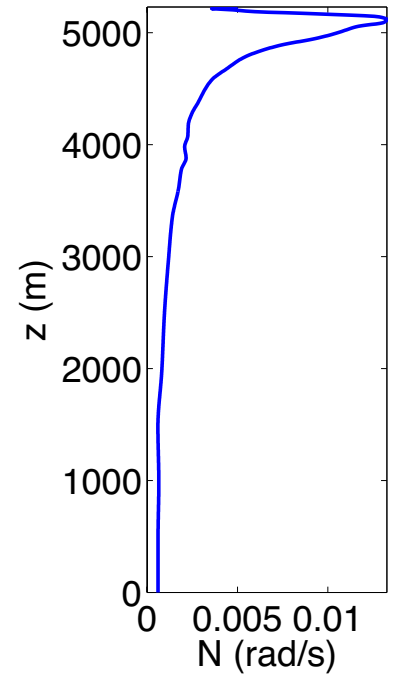

(b)

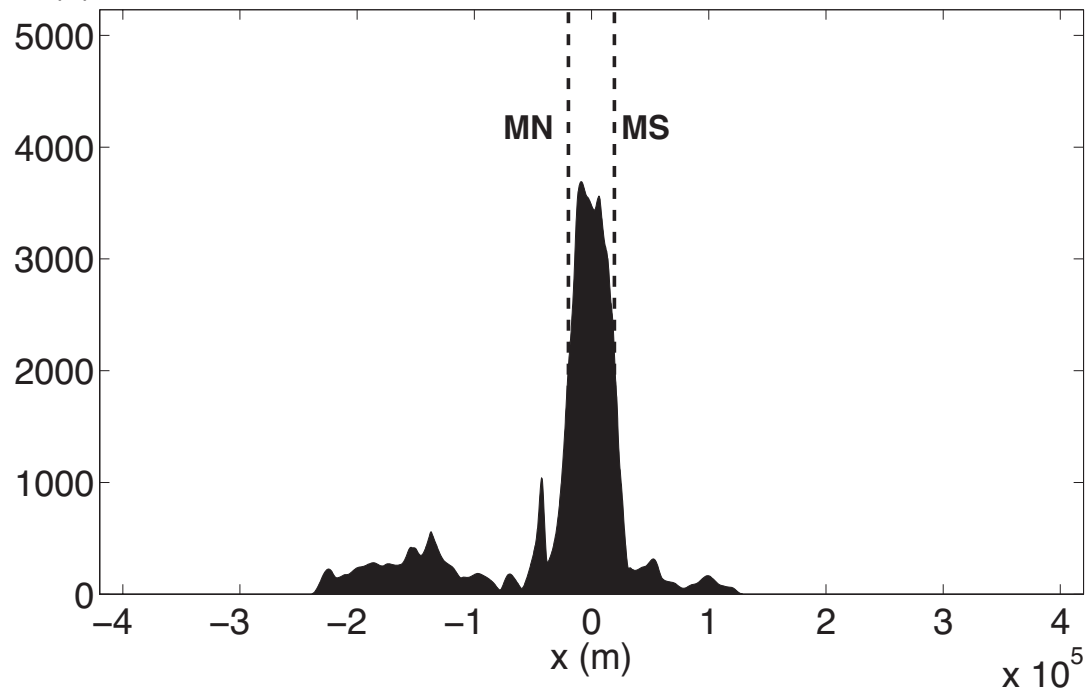

Figure 2-12: The $(a)$ stratification and (b) topography of the N-S transect of the Line Islands Ridge shown in figure 2-10. In (b) the dashed lines MN and MS indicate effective locations of mooring $\mathrm{N}$ and $\mathrm{S}$ from the EXITS study, respectively.

pographies have minimal small-scale features before and after a central peak, as well as comparable peak heights and widths. These similarities suggest that the resulting scattered and generated internal wave fields should be analogous to those predicted by the Green function solution of the Gaussian ridge.

\subsubsection{Generation results}

As in Section 2.3.1, this section seeks to quantify the internal tide generation at the Line Islands Ridge by examining the cases of the U-N and N-S transects shown in figure 2-10. With respect to the U-N transect, figure 2-13 presents the horizontal velocity field of the generated internal tide as predicted by Green function theory, normalized by the barotropic tidal velocity, $u_{0}$. From the velocity field, the increased number of wave beams upstream compared to downstream of the topography indicate that a greater amount of the generated internal tide propagates leftward of the topography.

This result further shown in figure 2-14 (a), where $64.7 \%$ of the energy flux of the generated internal tide propagates to the left. Furthermore, of the generated internal 


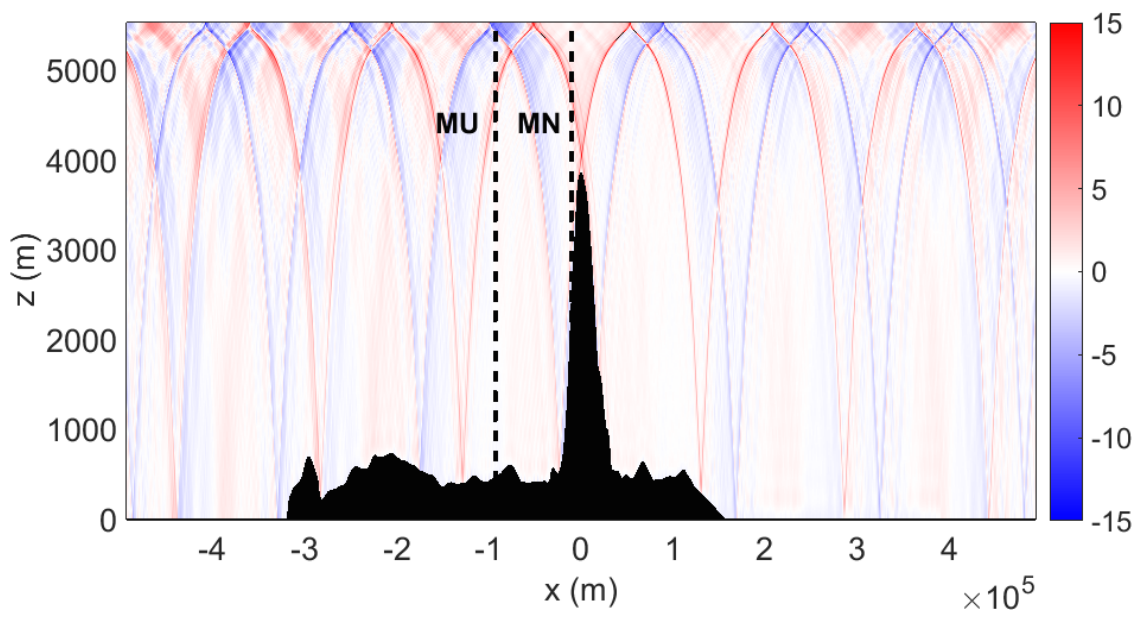

Figure 2-13: Normalized horizontal velocity field $\left(u(x, z, t) / u_{0}\right)$ of the internal tide at an arbitrary instant in time generated at the U-N transect shown in figure 2-10.

tide, leftward and rightward propagating mode- 1 internal tide accounts for $26.3 \%$ of the energy flux, and mode-2 for $40.7 \%$. While the U-N transect is a strong generator of mode- 2 internal tides, the majority of mode 2 generated, $67.9 \%$, propagates upstream of the topography.

Examining the energy flux at various locations along the U-N transect, as shown in figure 2-14(b), further demonstrates the similarities between the internal tide at locations MU and MN. Specifically, the energy flux at MU differs by about 5.5\% to
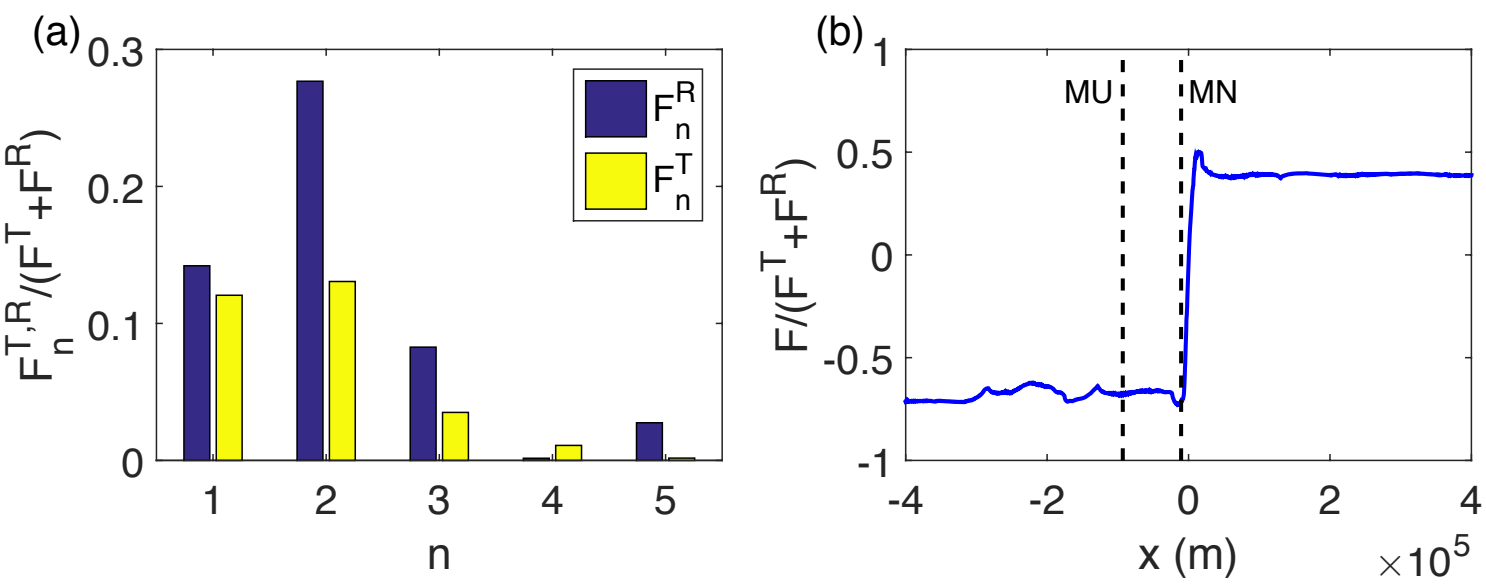

Figure 2-14: (a) Modal decomposition of the leftward $\left(F_{n}^{R}\right)$ and rightward $\left(F_{n}^{T}\right)$ propagating energy flux and $(b)$ the local depth-integrated, time-averaged, across-ridge energy flux of the internal tide generated at the U-N transect shown in figure 2-10. Both are normalized by the total energy flux. In (b), dashed lines MU and MN correspond to the locations of mooring $\mathrm{U}$ and $\mathrm{N}$ of the EXITS study, respectively. 
the far-field leftward propagating internal tide while the energy flux at MN differs by less than $1 \%$. This indicates that the generated internal tide at MU is unchanged in the far-field by the small scale topography. Furthermore, these results show the modal content of the internal tide incident on the U-N transect is virtually unchanged between mooring $\mathrm{U}$ and mooring $\mathrm{N}$.

As for the case of the Gaussian ridge studied in 2.3.1, figure 2-15 shows the modal content of the generated internal tide at various locations along the U-N transect using local topographic depth, 2-15(a), and far-field ocean depth, 2-15(b). As in the case of the internal tide generated at the Gaussian ridge, the modal content determined using the local depth and the far-field depth vary a great deal. Comparing the sum of the energy flux in all modes at MU when using the local depth to that computed by 2.30 and shown in figure 2-14(b) shows that the energy flux computed by modal decomposition differs by over $3.2 \%$. In the same way, the total energy flux at MN is 44.5\% less than the energy flux at MN in figure 2-14(b). However, the total energy flux at MU as computed using the far-field ocean depth, figure 2-15(b), is within $2.5 \%$ of that shown in figure 2-14(b). The total energy flux at $\mathrm{MN}$ is also in excellent

(a)

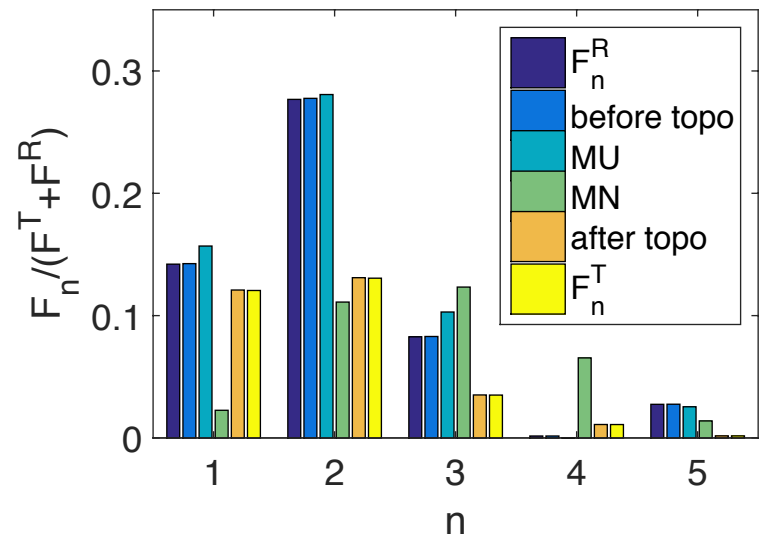

(b)

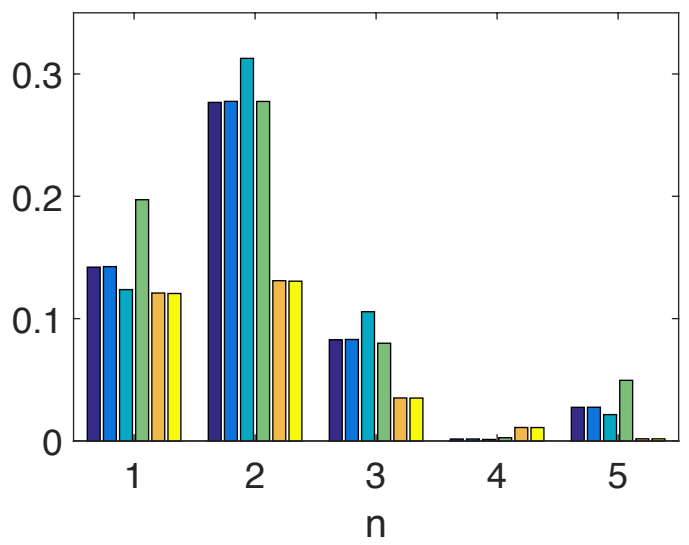

Figure 2-15: Modal decomposition of the net energy flux of the generated internal tide before, after, and at locations $\mathrm{MU}$ and $\mathrm{MN}$ on the topography of the $\mathrm{U}-\mathrm{N}$ transect shown in figure 2-11 normalized by the total energy flux. The profiles are decomposed using the $(a)$ local depth and $(b)$ far-field depth. The net energy flux in the far-field before the topography $\left(F_{n}^{R}\right)$ and after the topography $\left(F_{n}^{T}\right)$ are also plotted, and should roughly agree with the modal decomposition of the net energy flux at the profiles taken before and after the topography, respectively. 
agreement, with only $1.7 \%$ difference between the energy flux at MN in figure 2-14(b). These results further indicate that the far-field depth choice yields an accurate modal decomposition of the internal wave field.

Investigating the modal content determined using the far-field depth modal decomposition further, figure 2-15(b) shows that the modal decomposition of the generated internal tide before and after the topography is in excellent qualitative agreement with the modal content of the internal tides before and after the topography as determined by the Green function approach. Additionally, while the modal content of the internal tide at MU has a similar partition of energy flux to that before the topography, the mode 1 and mode 2 content differs by $12.9 \%$, and the mode 3 content differs by $27.8 \%$ to that of the far-field internal tide as predicted by the Green function method. Disagreements are also present when comparing the modal content at MN to that of the far-field energy flux after the topography, in which case modal decomposition predicts $38.8 \%$ greater mode 1 than the far-field energy flux before the topography. Additionally, the modal content measured at MU and MN differ greatly in their prediction of the energy flux in modes 1-3 and 5, in some cases by over $58 \%$. These results are in agreement with those for the case of the generated internal tide at a Gaussian topography, further suggesting that the modal content of an internal tide at locations along the topography may not be a good indication of the modal content of the far-field propagating internal tide.

Figure 2-16 shows the normalized horizontal velocity field of the internal tide generated at the N-S transect. The complexity of the wave beams both upstream and downstream of the topography suggest generated high-mode internal tides propagate both to the left and right of the topography.

As plotted in figure 2-17 (a), mode 1 accounts for $42.4 \%$ of the generated internal tide, whereas mode 2 accounts for $35.2 \%$. In the case of the leftward propagating internal tide, mode 1 and 2 each account for $\sim 40 \%$ of the energy flux. Furthermore, of the mode- 2 energy flux of the generated internal tide, the vast majority of it, $67 \%$, propagates upstream of the topography.

The energy flux along the N-S transect, presented in figure 2-17(b), indicates 


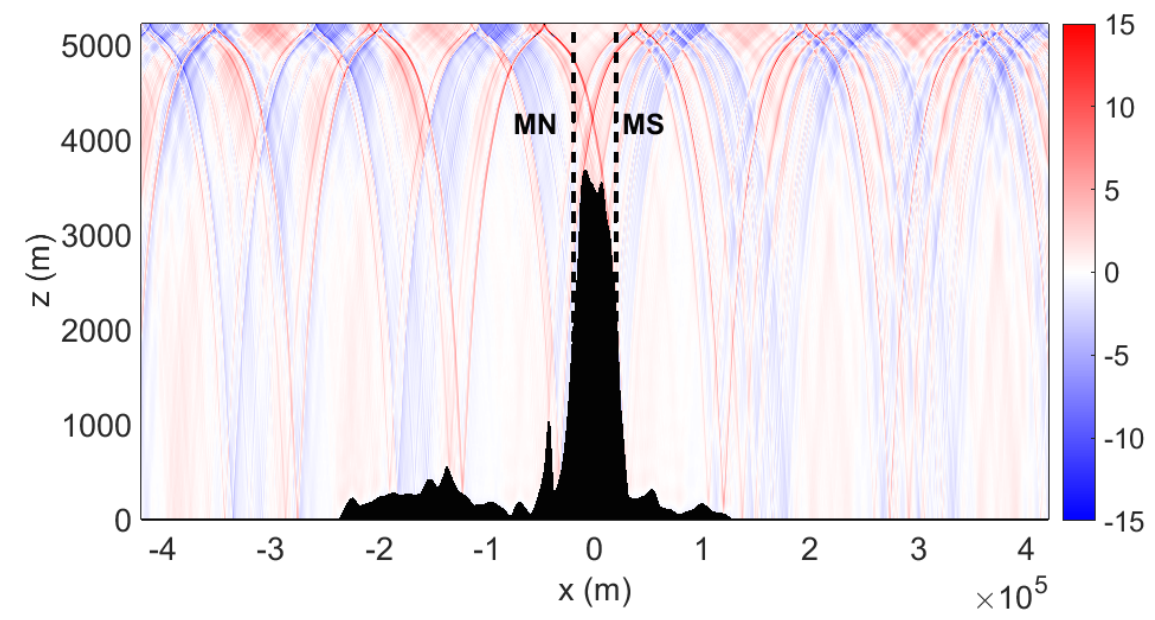

Figure 2-16: Normalized horizontal velocity field $\left(u(x, z, t) / u_{0}\right)$ at an arbitrary instant in time generated at the N-S transect shown in figure $2-10$.
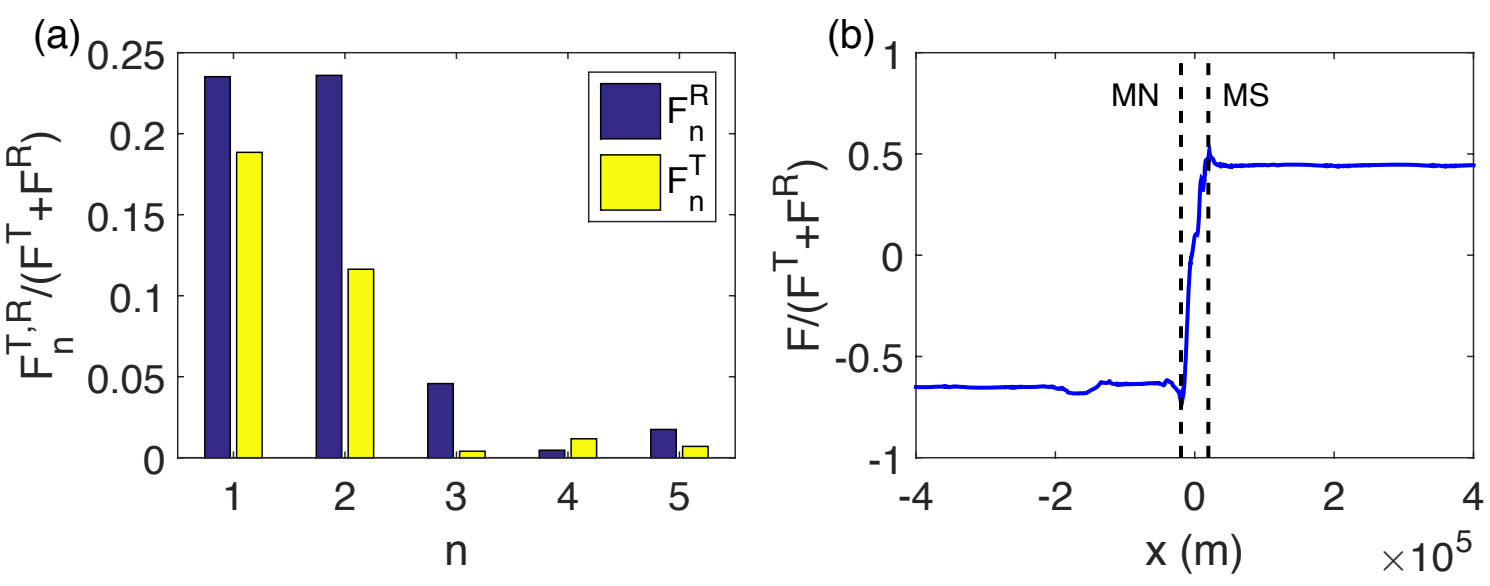

Figure 2-17: $(a)$ Modal decomposition of the leftward $\left(F_{n}^{R}\right)$ and rightward $\left(F_{n}^{T}\right)$ propagating energy flux and $(b)$ the local depth-integrated, time-averaged, across-ridge energy flux of the internal tide generated at the N-S transect shown in figure 2-10. Both are normalized by the total energy flux. In (b), dashed lines MN and MS correspond to the locations of mooring $\mathrm{N}$ and $\mathrm{S}$ of the EXITS study, respectively.

that the energy flux of the generated internal tide at $\mathrm{MN}$ is approximately $4.8 \%$ different from the energy flux of the far-field leftward propagating generated internal tide. Similarly, the energy flux at MS differs from the far-field rightward propagating generated internal tide by only about $6.8 \%$. These results further demonstrated that modal content of the internal tide as measured at locations MN or MS are representative of the far-field internal tide modal content.

The modal content of the generated internal tide at the N-S transect is further 
(a)

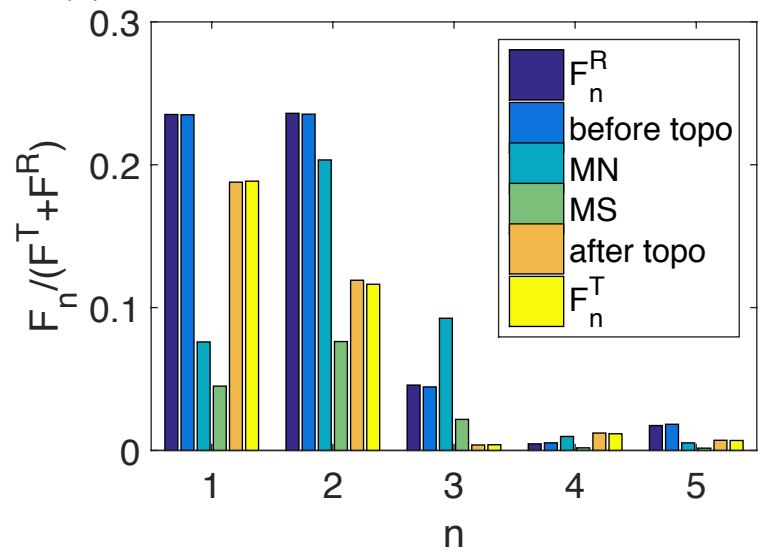

(b)

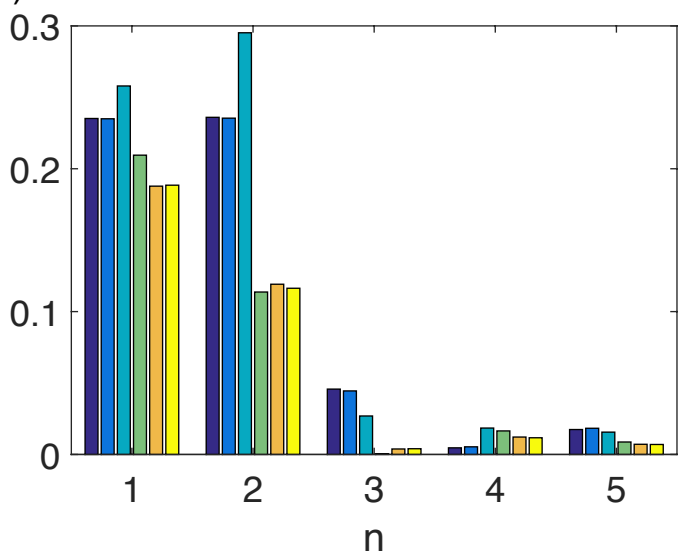

Figure 2-18: Modal decomposition of the net energy flux of the generated internal tide before, after, and at locations MN and MS on the topography of the N-S transect shown in figure 2-12 normalized by the total energy flux. The profiles are decomposed using the $(a)$ local depth and $(b)$ far-field depth. The net energy flux in the far-field before the topography $\left(F_{n}^{R}\right)$ and after the topography $\left(F_{n}^{T}\right)$ are also plotted, and should roughly agree with the modal decomposition of the net energy flux at the profiles taken before and after the topography, respectively.

investigated in figure 2-18. The sum of the energy flux in all modes at MN determined when using the local depth, figure 2-18( $(a)$, is $37.3 \%$ less than that of the energy flux at $\mathrm{MN}$ as determined by (2.30) and shown in figure 2-17(b). Similarly, the total energy flux at MS differs by $53 \%$ from that in 2-17(b). On the other hand, the total energy flux at MN as computed using the far-field depth, figure 2-18(b), is within $1 \%$ of that shown in figure 2-17(b), and the total energy flux at MS differs by only $7.9 \%$ from that at MS in figure 2-17(b). These results further emphasize that using the far-field ocean depth yields an accurate modal decomposition of a generated internal wave field.

The modal content determined using the far-field depth modal decomposition, figure 2-18(b), as in previous sections, shows excellent agreement between the modal decomposition of the generated internal tide before and after the topography and the modal content in the far-field as determined by the Green function method. The model partition of the energy flux at MN and MS is qualitatively similar to that of the far-field internal tide propagating to the left and right, respectively. The largest disparities between the energy flux over the topography and that in the far-field at 
MN are in mode 3, which differs by $41.1 \%$, and mode 4 , which differs by over $298 \%$. These differences are less pronounced at MS, with the largest difference between the energy flux of far-field internal tide after the topography of $11.1 \%$ in mode 1 . As in previous investigations of the modal content of the generated internal tide earlier in this chapter, these results further indicate that, for topographies of dominant internal tide generation, the modal content of an internal tide at locations along the topography may not be a good indication of the modal content of the far-field propagating internal tides.

\subsubsection{Scattering results}

Figure 2-19 presents a snapshot of the theoretical result for the incident-plus-scattered wave field resulting from a mode-1 internal tide propagating from the left to right. As in figure 2-7, the horizontal velocity has been normalized by the incident mode-1 internal tide velocity, $u_{0}$.

The modal decomposition of the transmitted and reflected energy flux normalized by the incident mode-1 energy flux for the U-N transect is shown in figure $2-20(a)$. Theory predicts that in this case, $78.2 \%$ of the incident mode- 1 energy is transmitted and the remaining $21.8 \%$ is reflected. Furthermore, $53.9 \%$ of the incident mode- 1

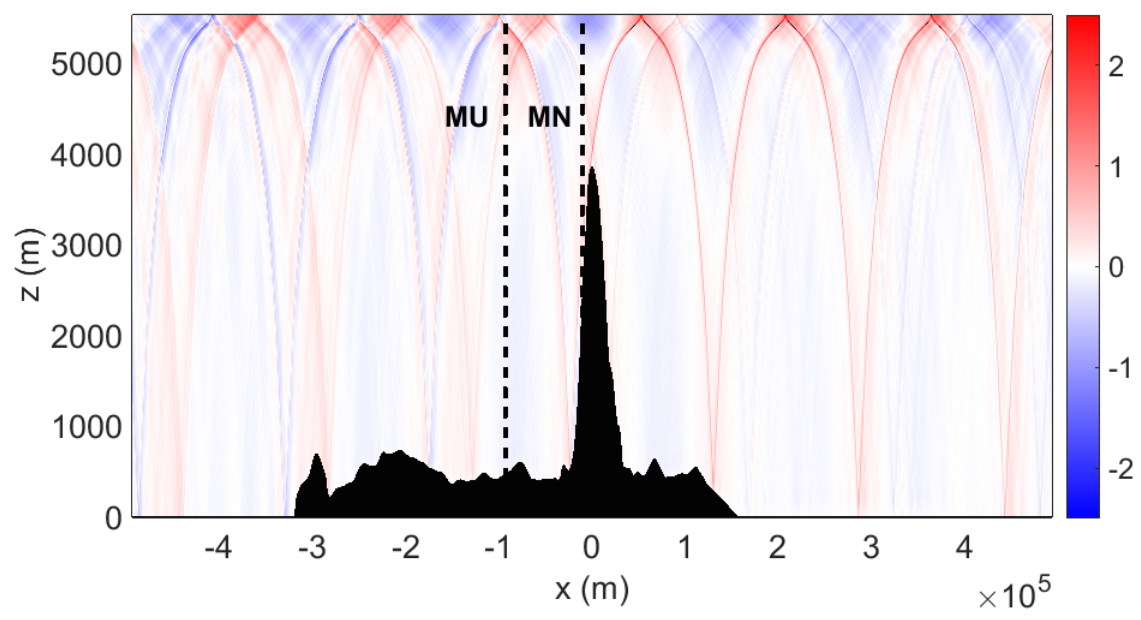

Figure 2-19: Normalized incident-plus-scattered horizontal velocity field $\left(u(x, z, t) / u_{0}\right)$ at an arbitrary instant in time for mode-1 (incident from the left) scattering from the U-N transect shown in figure 2-10. 

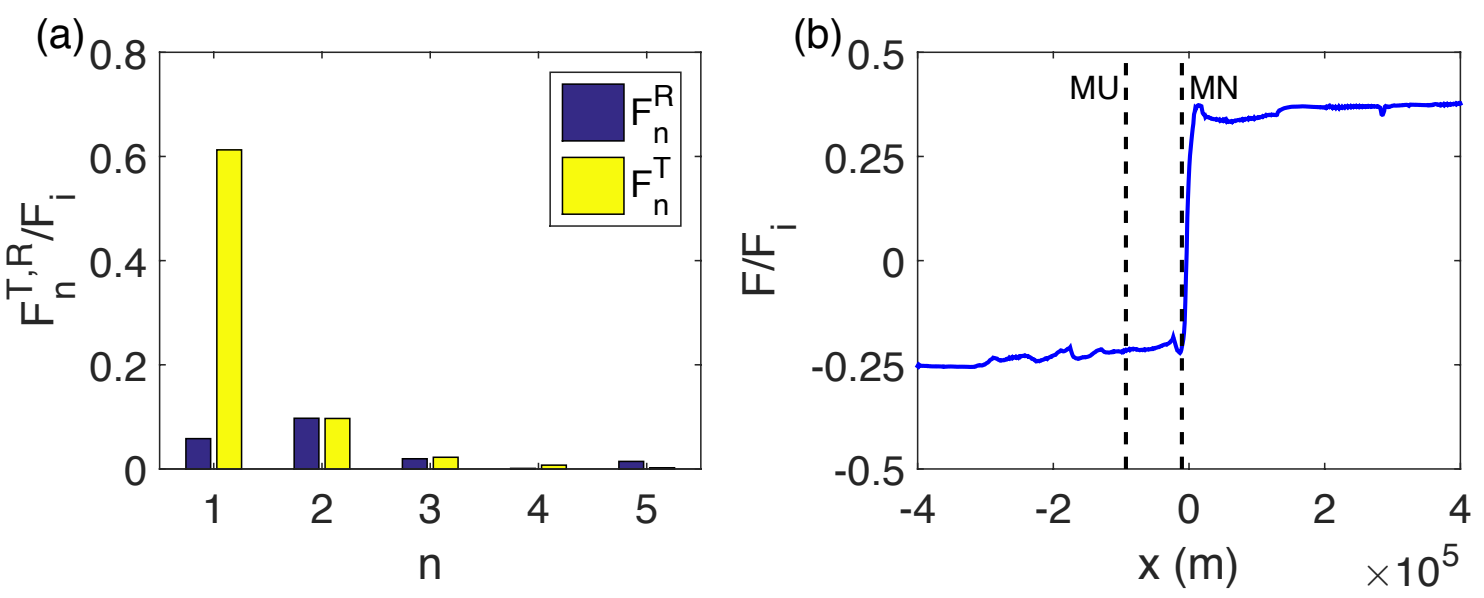

Figure 2-20: (a) Modal decomposition of reflected $\left(F_{n}^{R}\right)$ and the transmitted $\left(F_{n}^{T}\right)$ energy flux and $(b)$ the local depth-integrated, time-averaged, across-ridge energy flux of the internal tide scattered by the U-N transect shown in figure 2-10. Both are normalized by the incident mode- 1 energy flux. In (b), dashed lines MU and MN correspond to the locations of mooring $\mathrm{U}$ and $\mathrm{N}$ of the EXITS study, respectively.

energy is scattered into higher modes either in the transmitted or reflected wave field, suggesting that the topography at this transect is extremely efficient at scattering.

As seen in figure 2-20(b), the energy flux of the internal tide at MU differs from the far-field reflected energy flux by about $15.7 \%$, while at MN it differs by about $19.2 \%$. This suggests that the modal content at both MU and MN is significantly different from that of the far-field reflected internal tide. However, given that there is an incident mode-1 internal tide acting at both locations, the modal content at both $\mathrm{MU}$ and $\mathrm{MN}$ will be mode-1 dominated. The fact that energy flux between MU and MN differs by only approximately 3.5\% further indicates that the internal tide is not changing drastically between the two moorings. Therefore, the modal content extracted from velocity profiles at mooring $\mathrm{N}$ of the EXITS cruise should be comparable to the modal content of the internal tide measured at mooring $\mathrm{U}$.

The modal content at various locations along the U-N transect of the incidentplus-scattered internal tide is presented in figure 2-21. Motivated by the results from Section 2.4.1, it is expected that the modal decomposition technique when using the far-field ocean depth should yield correct results. As seen in the figure, unlike in the generation case, the modal content determined when using the local topographic 
(a)

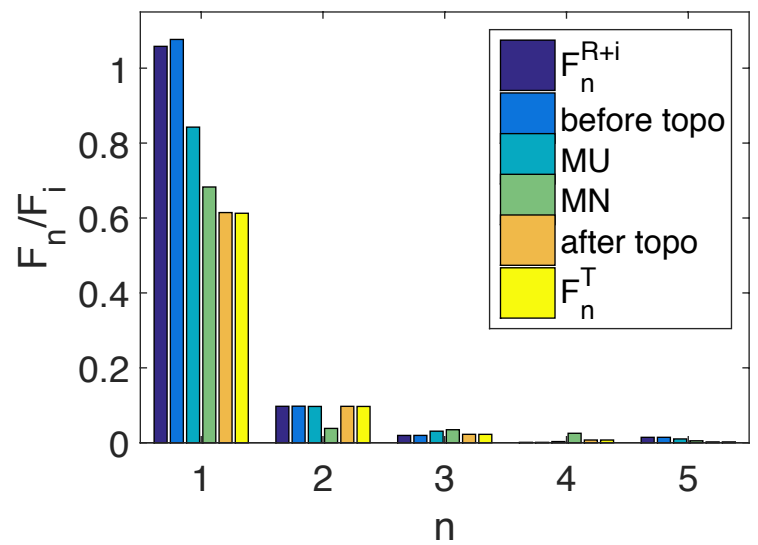

(b)

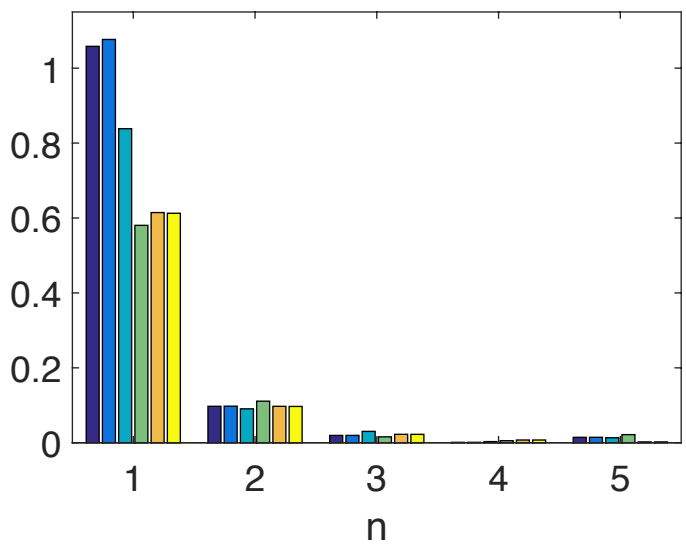

Figure 2-21: Modal decomposition of the net energy flux of the incident-plus-scattered internal tide before, after, and at locations $\mathrm{MU}$ and $\mathrm{MN}$ on the topography of the U-N transect shown in figure 2-11 normalized by the incident energy flux. The profiles are decomposed using the $(a)$ local depth and $(b)$ far-field depth. The energy flux before the topography $\left(F_{n}^{R+i}\right)$ and after the topography $\left(F_{n}^{T}\right)$ are also plotted, and should roughly agree with the modal decomposition of the net energy flux at the profiles taken before and after the topography, respectively.

depth, figure 2-21 (a) does not differ much from that determined when using the farfield depth, figure 2-21(b). Moreover, the distribution of the modal content at MU matches that of the energy flux before the topography, with the largest discrepancy in mode 1 with a difference of $\sim 20 \%$ for both methods. Likewise, the modal distribution of the energy flux of the internal tide at MN matches that of the far-field internal tide before the topography, differing the most only in mode 1 , for which the local topographic depth estimates the energy flux to be $35.5 \%$ less and the far-field ocean depth method estimates the energy flux to be $45.1 \%$ less than the far-field internal tide before the topography. As in the scattering results for the Gaussian topography presented in Section 2.3.2, these results suggest that, in contrast to the the generation case, the modal content at various locations along the topography is in good qualitative agreement with the far-field modal content. Furthermore, the depths used for the modal decomposition of a velocity field due to a scattered internal tide yield similar results and can thus be used interchangeably.

Figure 2-22 presents a snapshot of the normalized horizontal velocity theoretical results for the incident-plus-scattered wave field resulting from a mode-1 internal tide 


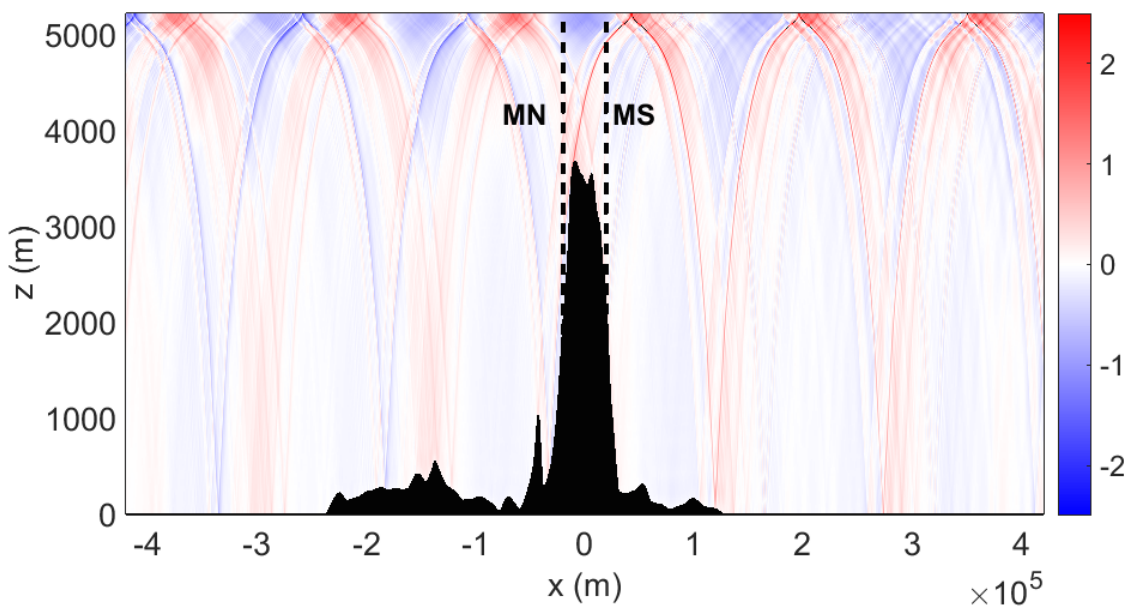

Figure 2-22: Normalized incident-plus-scattered horizontal velocity field $\left(u(x, z, t) / u_{0}\right)$ at an arbitrary instant in time for mode-1 (incident from the left) scattering the N-S transect shown in figure 2-10.

propagating from the left to right for the N-S transect. The presence of wave beams on both sides of the topography demonstrate the wave field is comprised of higher modes, indicating a scattered internal tide both transmitted and reflected.

Theory predicts that in the case of the N-S transect, $72.8 \%$ of the incident mode- 1 energy is transmitted and the remaining $27.2 \%$ is reflected, as shown in figure 223 (a). Furthermore, $39.8 \%$ of the incident mode- 1 energy is scattered into higher modes either in the transmitted or reflected wave field, suggesting that scattering by topography at this transect is substantial. As can be seen from figure 2-23( $a$ ), a greater amount of mode- 2 energy flux is transmitted than is reflected by the ridge.

Figure 2-23(b) shows that the energy flux at location MN differs only by about $3 \%$ from the far-field energy flux of the reflected internal tide. This suggests that in the plane of the N-S transect, the modal content of the internal wave field at MN is a good indication of the modal content of the energy flux of the reflected internal tide. Similarly, the transmitted far-field energy flux of the internal tide differs from the energy flux at MS by less than 4\%, further implying that the modal content of the scattered internal wave field at MN is representative of that of the far-field scattered internal wave field.

As done in for the case of the U-N transect, the modal content of the incident-plus- 

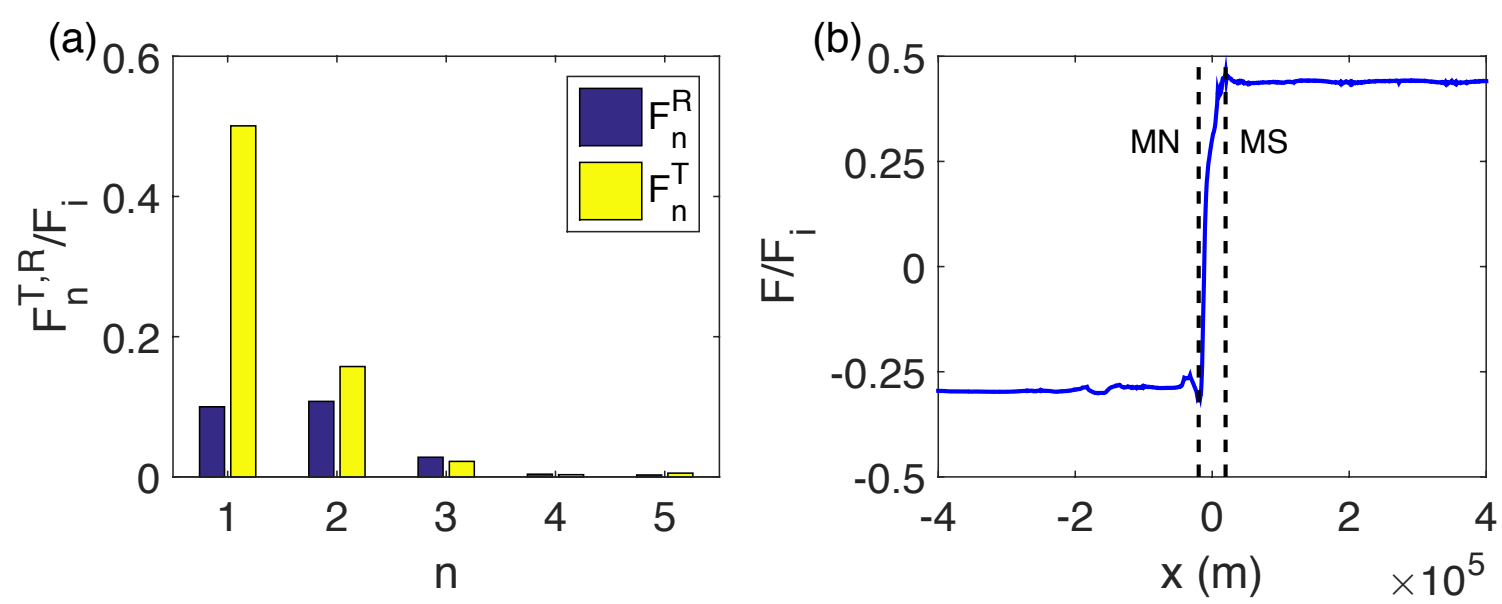

Figure 2-23: (a) Modal decomposition of the reflected $\left(F_{n}^{R}\right)$ and transmitted $\left(F_{n}^{T}\right)$ energy flux and $(b)$ the local depth-integrated, time-averaged, across-ridge energy flux of the internal tide scattered by the N-S transect shown in figure 2-10, Both are normalized by the incident mode-1 energy flux. In (b), dashed lines MN and MS correspond to the locations of mooring $\mathrm{N}$ and $\mathrm{S}$ of the EXITS study, respectively.

scattered internal tide field may also be investigated for the N-S transect, the results of which are shown in figure 2-24. Again, the far-field ocean depth method of modal decomposition is expected to yield accurate results. As seen in the case of the U-N

(a)

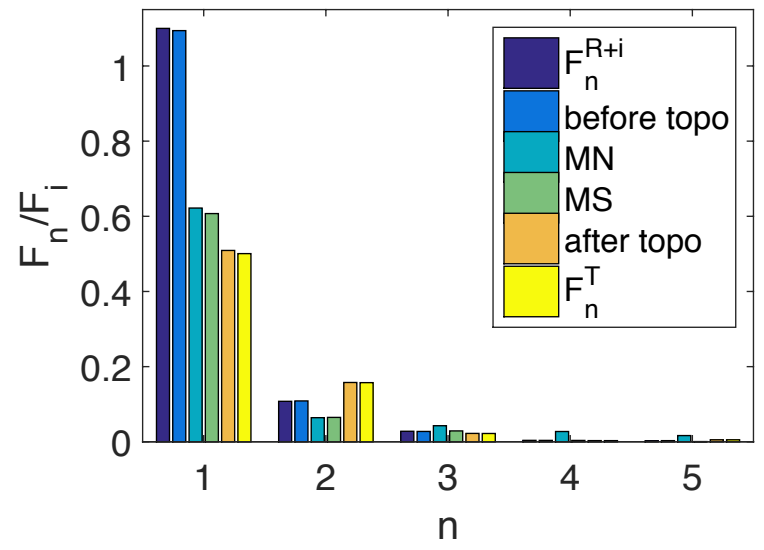

(b)

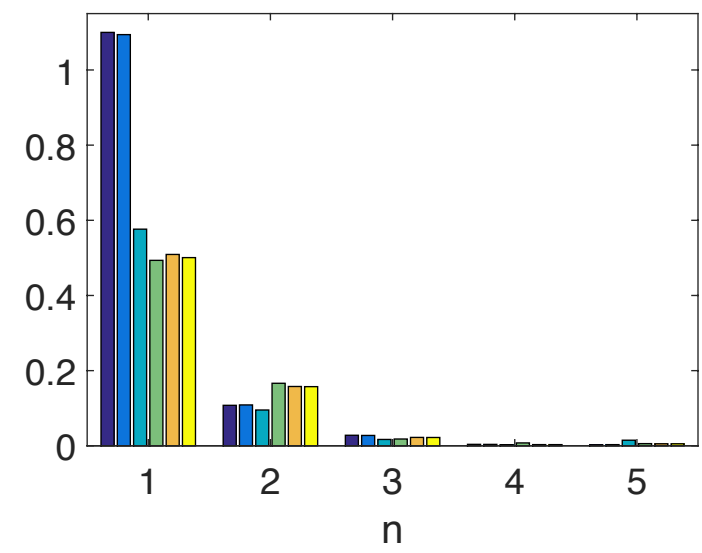

Figure 2-24: Modal decomposition of the net energy flux of the incident-plus-scattered internal tide before, after, and at locations $\mathrm{MN}$ and $\mathrm{MS}$ on the topography of the N-S transect shown in figure 2-12 normalized by the incident energy flux. The profiles are decomposed using the $(a)$ local depth and $(b)$ far-field depth. The energy flux before the topography $\left(F_{n}^{R+i}\right)$ and after the topography $\left(F_{n}^{T}\right)$ are also plotted, and should roughly agree with the modal decomposition of the net energy flux at the profiles taken before and after the topography, respectively. 
transect, again there is little difference between the modal content determined when using the local topographic depth, figure 2-24(a), and that determined when using the far-field depth, figure 2-24(b). While at MN and MS the distribution of modal content qualitatively matches that of the energy flux before and after the topography, respectively, there are large quantitative differences in the predicted modal energy flux. In particular, the mode 1 energy flux is $43.5 \%$ less than the energy flux of the internal tide before the topography when using the local depth modal decomposition, and $47.6 \%$ less then using the far-field ocean depth modal decomposition. This trend is also seen at MS when determining the modal content using local depth, for which the energy flux in mode 1 is $21.3 \%$ greater than that of the energy flux of the farfield transmitted internal tide and in mode 2 is $58.7 \%$ less. As with the modal decomposition study of the scattering case at the U-N transect, these results further confirm that the choice of depth for the modal decomposition of a velocity field due to a scattered internal tide is inconsequential. Furthermore, unlike the generation case, the modal content at locations along the topography is in good qualitative agreement with the modal content in the far-field.

These results are key to understanding the analysis of data from the EXITS cruise. They indicate that in the case of an incident mode- 1 internal tide scattered by the Line Islands Ridge, the modal parttion of energy flux as determined from measurements at mooring $\mathrm{U}$ and $\mathrm{N}$ should be the same. Therefore, if the measurements at mooring $\mathrm{U}$ indicate a mode-1 dominated internal wave field, it can be safely assumed that that mode-1 wave field encounters mooring $\mathrm{N}$ virtually unchanged. Additionally, the results at MS suggest that a modal decomposition of the wave field at mooring $\mathrm{S}$ will be analogous of the modal content of the far-field transmitted internal tide. Hence, if measurements taken at mooring $\mathrm{S}$ indicate larger amounts of mode-2 than at mooring $\mathrm{N}$, it can be further be reasoned that this mode- 2 dominated internal tide propagates into the far-field without being locally dissipated, thereby implying that this portion of the Line Islands Ridge is a topographic feature with strong internal tide scattering properties. Additionally, these results indicate that for a topographic feature that is a strong internal tide scatterer, such as the Line Islands Ridge, the choice of depth 
used in the modal decomposition of the internal tide at locations on the topography is insignificant. This assumption will be used in Chapter 4 where data from the EXITS cruise will be modally decomposed using the local topographic depth.

\subsection{Discussion and Conclusions}

Section 2.4 demonstrated that the topographies of the U-N and N-S transect of the Line Islands Ridge were efficient at both generating and scattering internal tides. The key question, however, is how strong is the scattering process compared to the generation process at each of these topographies? As in Section 2.3.3, characteristic values of the incident mode- 1 internal tide velocity, $u_{0}=0.05 \mathrm{~m} / \mathrm{s}$, and the barotropic tidal velocity, $u_{b}=0.03 \mathrm{~m} / \mathrm{s}$, at the Line Islands Ridge were used to determine the dimensional energy flux due to the scattered and generated internal tide at both the U-N and N-S transects. Specifically, the mode-2 energy flux of the scattered versus the generated internal tide at the Line Islands Ridge is examined. Table 2.1 summarizes the mode- 2 energy flux of the scattered and generated internal tides at the $\mathrm{U}-\mathrm{N}$ and N-S transects.

\begin{tabular}{|c|c|c|c|}
\hline Transect & Type & $E_{2}^{T}(\mathrm{~kW} / \mathrm{m})$ & $E_{2}^{R}(\mathrm{~kW} / \mathrm{m})$ \\
\hline U-N & Generated & 1.95 & 4.14 \\
& Scattered & 0.11 & 0.11 \\
\hline N-S & Generated & 1.90 & 3.86 \\
& Scattered & 0.18 & 0.12 \\
\hline
\end{tabular}

Table 2.1: Mode-2 energy flux of the internal tide produced by generation and scattering of an incident mode- 1 internal tide at the U-N and N-S transects.

The Green function approach predicts that in the case of both transects, the mode-2 energy flux of the internal tide produced by the scattering of an incident mode- 1 internal tide is an order of magnitude less than that produced by the generation of the internal tide at the topography. However, studies using three-dimensional numerical models and satellite altimetry (Johnston et al., 2003, Zhao et al., 2011) indicate that higher modes from internal tide scattering dominate at the Line Islands 


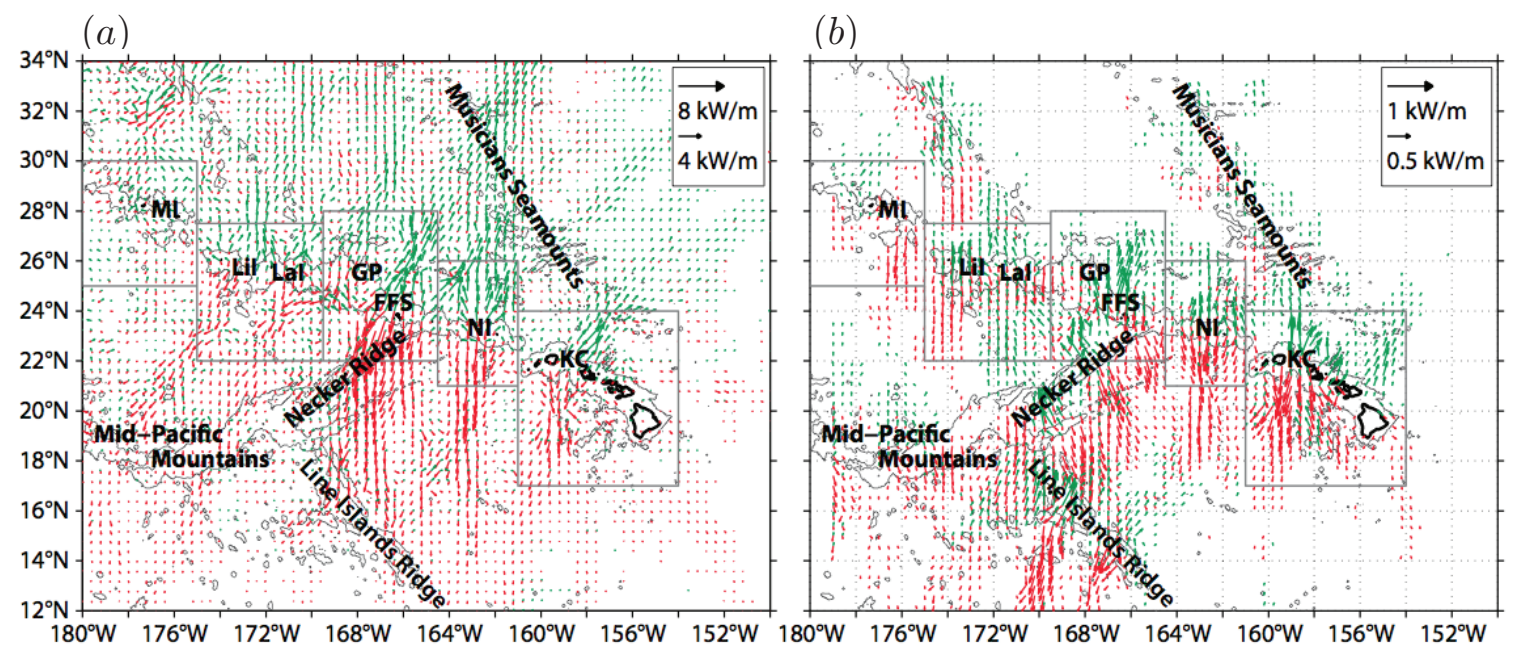

Figure 2-25: (a) Energy flux of mode-1 $M_{2}$ internal tides around the Hawaiian ridge, estimated from multisatellite altimetry using a $120 \mathrm{~km}$ fitting region. The $4,000 \mathrm{~m}$ isobath contours are shown in gray. Red and green arrows indicate southward and northward internal tides, respectively. Flux arrows smaller than $0.15 \mathrm{~kW} / \mathrm{m}$ are not plotted. (b) Mode- $2 M_{2}$ internal tides. Flux arrows smaller than $0.05 \mathrm{~kW} / \mathrm{m}$ are not plotted. Adapted from Zhao et al. (2011).

Ridge and does not appear to be a dominant local high-mode internal tide generator as mode 1 is diminished south of the ridge while mode 2 is augmented (figure 2-25), which was not predicted by the Green function approach.

While the predictions of the percentage of scattered internal tide energy flux align well with previous analytical, two- and three-dimensional numerical studies of the Line Islands Ridge (Mathur et al., 2014, Johnston et al., 2003), the relative value of highmode internal tide production via scattering versus generation at the ridge has not been investigated. The fact that previous studies indicate a significant diminishing of mode 1 at the Line Islands Ridge suggests that the assumption of a two-dimensional internal tide generation/scattering process is an oversimplification of the physical system.

\subsection{Future Work}

The results of this chapter indicate that there is a large uncertainty in determining the depth that should be used when modally decomposing internal tides. Preliminary 
studies indicate that the far-field ocean depth yields more accurate results than the local topographic depth for cases of internal tide generation, whereas for studies of internal tide scattering, the choice of depth is inconsequential to the results. Further work should be done to investigate the choice of depth in the accurate determination of modal content for topographies of varying criticality and height ratio.

Furthermore, this chapter began to study the differences between the modal content at locations on the topographic feature and the far-field internal tide. Initial results demonstrated that there is generally good qualitative agreement in modal partition of energy flux of the far-field internal tide and the internal tide along locations of the topography for cases of internal tide generation and scattering. Additional work should be done to explore for which classes of topographies, in terms of height ratio and criticality, the modal content of the internal tide above the topography is in good quantitative agreement with the far-field internal tide. Supplementary studies may also be conducted to determine the validity in utilizing flat-bottom modes for the modal decomposition of wave fields above varying topographic features with the aim of determining when and how flat-bottom modes may be implemented to determine the modal content of an internal wave field. 


\section{Chapter 3}

\section{Modal Decomposition of Internal Wave Fields}

\subsection{Introduction}

This chapter seeks to compare two methods for determining the modal composition of an internal wave field. The first is a projection method introduced in Section 1.3 and expanded in Section 3.2 allowing for a more general formulation that still converges with missing information in the vertical domain. The second is a regression technique widely used by physical oceanographers described in Section 3.3. Both of these methods are successful in determining the modal content of an internal wave field in the case of full-depth vertical information, as described in Section 3.4 . Thus, the focus of this chapter is to determine the effectiveness of each of these methods for cases of incomplete vertical domain, also described Section 3.4. The goal of this chapter is to determine the optimal algorithm for the modal decomposition of internal wave fields from the EXITS cruise (the results of which are discussed in Chapter 4), for which the three moorings deployed experienced various amounts of image loss in their measured horizontal velocity and isopycnal displacement. To this end, the performance of both modal decomposition algorithms is tested in Section 3.5 for an internal tide wave field at the Line Islands Ridge, as predicted by the Green function, with image loss consistent with the EXITS cruise. Finally, the chapter concludes with 
some examination into the number of modes required for both algorithms to converge and suggestions for future studies in Section 3.6 .

\subsection{Projection method}

This section presents a new analytical method to modally decompose vertical timeseries of field data that has some advantages over the current regression method. In particular, the new approach, referred to as the projection method, can accommodate a greater amount of data loss and has no ambiguity in choosing how many modes should be fit to the observed data set. The basis of the projection method was first introduced by Echeverri et al. (2009) and was further developed by Saidi (2011).

This system consists of two classes of variables: those that are directly related to the vertical structure of streamfunction, $\phi(z)$, and those that are related to its derivative, $d \phi(z) / d z$. The variables that are linearly related to $\phi(z)$ are given by:

$$
\left\{\begin{array}{l}
w \\
\eta \\
\rho
\end{array}\right\}=\phi(z)\left\{\begin{array}{c}
i k \\
-k / \omega \\
-\rho_{0} k N^{2} /(\omega g)
\end{array}\right\} e^{i(k x-\omega t)}
$$

where $w$ is the vertical velocity, $\eta$ is the isopycnal displacement, and $\rho$ is the density perturbation. The remaining class of variables proportional to $d \phi(z) / d z$ are:

$$
\left\{\begin{array}{l}
u \\
v \\
p
\end{array}\right\}=\frac{d \phi(z)}{d z}\left\{\begin{array}{c}
-1 \\
i f / \omega \\
\rho_{0}\left(f^{2}-\omega^{2}\right) /(k \omega)
\end{array}\right\} e^{i(k x-\omega t)},
$$

where $u$ and $v$ are the components of the horizontal velocity and $p$ is the pressure perturbation.

In oceanographic field studies, it is often impractical to analyze $w$ as vertical velocities are extremely small and very susceptible to instrument noise. Therefore, a much more reliably measured variable related to $\phi(z)$ is the isopycnal displacement $\eta$. Since the goal of this chapter is to determine the optimum algorithm to analyze the 
modal content of the internal wave fields measured by EXITS, an oceanographic field study, $u$ and $\eta$ are the two variables of interest. Hence, the details of the algorithm are presented first with respect to the horizontal velocity field, $u$, with the necessary changes to cross over to the isopycnal displacement, $\eta$, discussed after.

For a given stratification $N(z)$, the homogenous differential equation governing the vertical structure $\phi(z)$ of an internal wave is given by (1.22). Together with the homogenous boundary conditions of a flat bottom and a rigid lid, $\phi(z=0)=\phi(z=$ $H)=0$, equation 1.22 represents a Sturm-Liouville problem of the canonical form:

$$
\frac{d}{d z}\left[p(z) \frac{d g(z)}{d z}\right]+\left(q(z)+\lambda^{2} \sigma(z)\right) g(z)=0
$$

where $g(z)$ is the primary function of arbitrary nature, $p(z), q(z)$ and $\sigma(z)$ are given functions of $z$, and $\lambda$ is the eigenvalue to be determined. In the case of internal waves, the eigenvalue $\lambda=k$. Comparing (3.3) with (1.22) yields $g(z)=\phi(z), p(z)=1$, $q(z)=0$, and $\sigma(z)=\frac{N^{2}(z)-\omega^{2}}{\omega^{2}-f^{2}}$.

Focusing on the eigenvalues $\lambda_{m}$ and $\lambda_{n}$ and the corresponding eigenfunctions $g_{m}(z)$ and $g_{n}(z)$ that satisfy

$$
\frac{d}{d z}\left[p(z) \frac{d g_{m}(z)}{d z}\right]+\left(q(z)+\lambda_{m}^{2} \sigma(z)\right) g_{m}(z)=0
$$

and

$$
\frac{d}{d z}\left[p(z) \frac{d g_{n}(z)}{d z}\right]+\left(q(z)+\lambda_{n}^{2} \sigma(z)\right) g_{n}(z)=0
$$

proceeding towards determining the orthogonality relation, multiplying (3.4) by $g_{n}(z)$ and (3.5) by $g_{m}(z)$ and taking their difference yields:

$$
g_{n}(z) \frac{d}{d z}\left[p(z) \frac{d g_{m}(z)}{d z}\right]-g_{m}(z) \frac{d}{d z}\left[p(z) \frac{d g_{n}(z)}{d z}\right]+\left(\lambda_{m}^{2}-\lambda_{n}^{2}\right) g_{m}(z) g_{n}(z) \sigma(z)=0
$$

Integrating (3.6) over the interval $[a, b]$ results in 


$$
\int_{a}^{b} g_{m}(z) g_{n}(z) \sigma(z) \mathrm{d} z=\delta_{m n}
$$

where $\delta_{m n}$ is a Kronecker delta. Equation (3.7) states that the eigenfunctions of the Sturm-Liouville problem are orthogonal to each other and form a complete set of basis functions with which to describe any function over $[a, b]$. For $q(z)=0$, as is the case for (1.22), multiplying (3.4) by $g_{n}(z)$ and integrating over the interval $[a, b]$ yields:

$$
\int_{a}^{b} \frac{d g_{m}(z)}{d z} \frac{d g_{n}(z)}{d z} \mathrm{~d} z=\delta_{m n}
$$

which states that the derivatives of the eigenfunctions are also orthogonal to each other and form a basis.

By relations (3.1) and (3.2) and equations (3.7) and (3.8), the orthogonality relations for isopycnal displacement $\eta$, and the horizontal velocity $u$, over the domain $z \in[0, H]$ are

$$
\int_{0}^{H} \hat{\eta}_{m}(z) \hat{\eta}_{n}(z)\left(\frac{N^{2}(z)-\omega^{2}}{\omega^{2}-f^{2}}\right) d z=\delta_{m n}
$$

and

$$
\int_{0}^{H} \hat{u}_{m}(z) \hat{u}_{n}(z) d z=\delta_{m n}
$$

where $\hat{\eta}(z)=-k_{n} \phi_{n}(z) / \omega$ and $\hat{u}(z)=-d \phi_{n}(z) / d z$ are the vertical structures of the isopycnal displacement ahd horizontal velocities, respectively. Furthermore, these modes form a complete basis such that the linear wave field for the isopycnal disaplcement and horizontal velocity can be expressed as

$$
\eta(x, z, t)=\sum_{n=1}^{\infty} \Re\left\{\mathcal{H}_{n} \hat{\eta}_{n} e^{i\left(k_{n} x-\omega t\right)}\right\}
$$

and

$$
u(x, z, t)=\sum_{n=1}^{\infty} \Re\left\{\mathcal{U}_{n} \hat{u}_{n} e^{i\left(k_{n} x-\omega t\right)}\right\},
$$

where $\mathcal{H}_{n}$ and $\mathcal{U}_{n}$ are the complex coefficients describing the amplitude and phase of 
each mode for the isopycnal displacement and horizontal velocity respectively.

\section{Full depth information}

The projection method exploits the orthogonality relation of internal wave modes to determine weighting factors for each mode based on the extent of the data available. First, the straightforward case of horizontal velocity field data across the full depth of the water column $z \in[0, H]$, is considered. In this case the orthogonality relation (3.10) may be readily exploited to determine the modal content of the internal wave field as follows. For a pair of profiles collected at $x_{0}$ at times $t_{1}$ and $t_{2}$, making use of equation 3.12 results in:

$$
\begin{aligned}
& \int_{0}^{H} u\left(x_{0}, z, t_{1}\right) \hat{u}_{m}(z) d z=\Re\left\{\mathcal{U}_{m} e^{i\left(k_{m} x_{0}-\omega t_{1}\right)}\right\} \Upsilon, \\
& \int_{0}^{H} u\left(x_{0}, z, t_{2}\right) \hat{u}_{m}(z) d z=\Re\left\{\mathcal{U}_{m} e^{i\left(k_{m} x_{0}-\omega t_{2}\right)}\right\} \Upsilon,
\end{aligned}
$$

where by definition $\Upsilon=\int_{0}^{H} \hat{u}_{m}^{2}(z) d z=1$. Equating the real and imaginary parts of $\mathcal{U}_{m}$ from (3.13) and (3.14) and rearranging yields:

$$
\begin{aligned}
{\left[\begin{array}{l}
\Re\left\{\mathcal{U}_{m}\right\} \\
\Im\left\{\mathcal{U}_{m}\right\}
\end{array}\right] } & =\left[\begin{array}{cc}
-\cos \left(k_{m} x_{0}-\omega t_{2}\right) & \cos \left(k_{m} x_{0}-\omega t_{1}\right) \\
\sin \left(k_{m} x_{0}-\omega t_{2}\right) & -\sin \left(k_{m} x_{0}-\omega t_{1}\right)
\end{array}\right] \\
& \times(\sin (\omega \Delta t) \Upsilon)^{-1} \int_{0}^{H} \hat{u}_{m}(z)\left[\begin{array}{l}
u\left(x_{0}, z, t_{1}\right) \\
u\left(x_{0}, z, t_{2}\right)
\end{array}\right] d z,
\end{aligned}
$$

which is well defined for $\Delta t \neq \pi / \omega,(p=\mathbb{Z} \geq 0)$. The mode strength and phase are, respectively:

$$
\begin{aligned}
\left|\mathcal{U}_{m}\right| & =\sqrt{\Re\left\{\mathcal{U}_{m}\right\}^{2}+\Im\left\{\mathcal{U}_{m}\right\}^{2}}, \\
\varphi_{m}^{(u)} & =\arctan \left(\frac{\Im\left\{\mathcal{U}_{m}\right\}}{\Re\left\{\mathcal{U}_{m}\right\}}\right) .
\end{aligned}
$$


For the case of the isopycnal displacements, the complex coefficient $\mathcal{H}_{m}$ can be obtained from $\eta$ field data via the same process outlined above by alternatively using the basis functions $\hat{\eta}_{m}(z)$ and orthogonality relation $(3.9)$, in which case $\Upsilon=$ $\int_{0}^{H} \hat{\eta}_{m}^{2}(z)\left(\frac{N^{2}(z)-\omega^{2}}{\omega^{2}-f^{2}}\right) d z=1$, i.e.

$$
\begin{gathered}
{\left[\begin{array}{l}
\Re\left\{\mathcal{H}_{m}\right\} \\
\Im\left\{\mathcal{H}_{m}\right\}
\end{array}\right]=\left[\begin{array}{cc}
-\cos \left(k_{m} x_{0}-\omega t_{2}\right) & \cos \left(k_{m} x_{0}-\omega t_{1}\right) \\
\sin \left(k_{m} x_{0}-\omega t_{2}\right) & -\sin \left(k_{m} x_{0}-\omega t_{1}\right)
\end{array}\right]} \\
\times(\sin (\omega \Delta t) \Upsilon)^{-1} \int_{0}^{H} \hat{\eta}_{m}(z)\left[\begin{array}{l}
\eta\left(x_{0}, z, t_{1}\right) \\
\eta\left(x_{0}, z, t_{2}\right)
\end{array}\right]\left(\frac{N^{2}(z)-\omega^{2}}{\omega^{2}-f^{2}}\right) d z .
\end{gathered}
$$

The magnitude and phase of the horizontal velocity and isopyncal perturbation are related by

$$
\left|\mathcal{H}_{m}\right|=\frac{k_{m}}{\omega}\left|\mathcal{U}_{m}\right|
$$

and

$$
\varphi_{m}^{(\eta)}=\varphi_{m}^{(u)}
$$

\section{Incomplete vertical domain}

In any field data set it is the case that there will be incomplete data over the vertical domain. Acoustic Doppler current profilers, for example, cannot reliably measure velocities near the sea floor due to the limited number of deep scatterers, and data from mooring deployments can begin significantly beneath the ocean surface, resulting in data loss at the top and bottom of the domain. Crawling instruments such as the commonly used McLane moored profilers (such as those used in the EXITS study) cannot traverse the entire water column at speeds necessary for the detection of internal waves (Doherty et al. 1999$)$. Therefore, they are often used stacked in pairs,

collecting data is regions of the upper and lower water column (Alford, 2010, Johnston et al. 2013); this arrangement also has data loss in the region between the domains of the two profilers. 


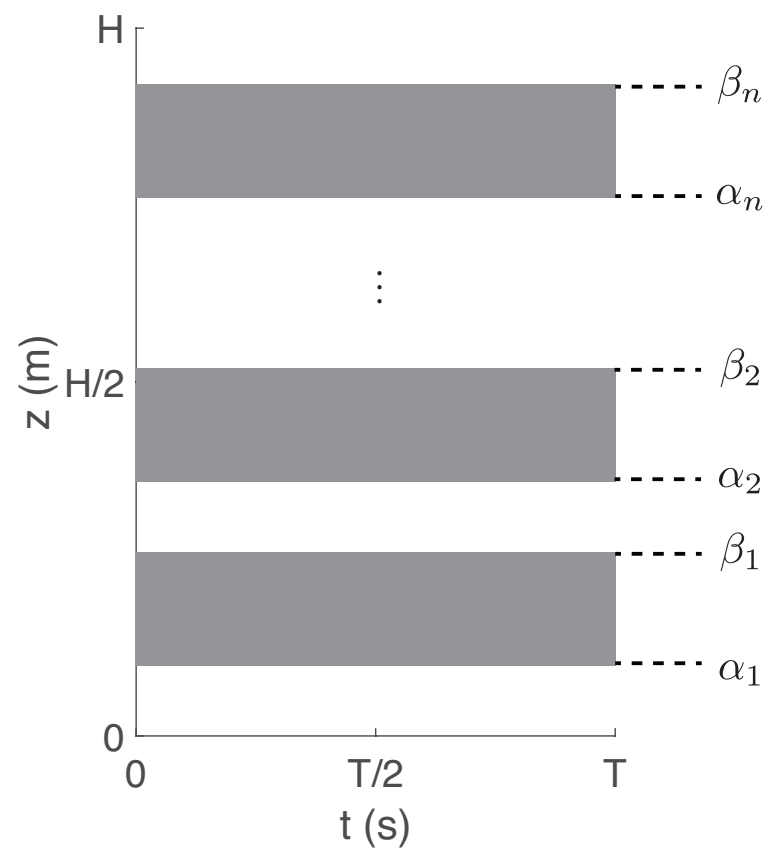

Figure 3-1: Example of oceanographic data with image loss throughout the vertical domain.

The modal projection method can be modified to account for this data loss. Let $z \in\left[\alpha_{i}, \beta_{i}\right]$ be the $i$ vertical domains for which data is available (as illustrated in figure $3-1$, where $\left[\alpha_{i}, \beta_{i}\right] \subset[0, H]$ and $i=1, \ldots, q$. The vertical modes are no longer orthogonal over the composition of these subdomains, and hence from equations (3.10) and 3.12 :

$$
\sum_{i=1}^{q} \int_{\alpha_{i}}^{\beta_{i}} u\left(x_{0}, z, t\right) \hat{u}_{m}(z) d z=\sum_{n=0}^{\infty} \Gamma_{n}\left(x_{0}, t\right) \Upsilon_{m n}
$$

where

$$
\begin{aligned}
\Upsilon_{m n} & =\sum_{i=1}^{q} \int_{\alpha_{i}}^{\beta_{i}} \hat{u}_{m}(z) \hat{u}_{n}(z) d z, \\
\Gamma_{n} & =\Re\left\{\mathcal{U}_{n} e^{i\left(k_{n} x_{0}-\omega t\right)}\right\},
\end{aligned}
$$

If a wave field is assumed to comprise modal content up to mode $M$, implementing equation (3.21) for modes $n=1$ through $n=M$, then results in the linear problem: 


$$
\boldsymbol{\Gamma}\left(x_{0}, t\right)=\mathbf{\Upsilon}^{-1} \times \sum_{i=1}^{q} \int_{\alpha_{i}}^{\beta_{i}} u\left(x_{0}, z, t\right)\left[\begin{array}{c}
\hat{u}_{1}(z) \\
\vdots \\
\hat{u}_{M}(z)
\end{array}\right] d z
$$

where $\boldsymbol{\Gamma}$ is an $M \times 1$ matrix whose elements are given by 3.23 and $\Upsilon$ is an $M \times M$ matrix with elements given by 3.22 that reduces to the identity matrix in the limit of full depth information. Recovering the real and imaginary components of $\mathcal{U}_{m}$ is achieved by implementing this procedure at two different times, $t_{1}$ and $t_{2}$, and using the relation:

$$
\left[\begin{array}{l}
\Re\left\{\mathcal{U}_{m}\right\} \\
\Im\left\{\mathcal{U}_{m}\right\}
\end{array}\right]=(\sin (\omega \Delta t))^{-1}\left[\begin{array}{cc}
-\cos \left(k_{m} x_{0}-\omega t_{2}\right) & \cos \left(k_{m} x_{0}-\omega t_{1}\right) \\
\sin \left(k_{m} x_{0}-\omega t_{2}\right) & -\sin \left(k_{m} x_{0}-\omega t_{1}\right)
\end{array}\right]\left[\begin{array}{c}
\Gamma_{m}\left(x_{0}, t_{1}\right) \\
\Gamma_{m}\left(x_{0}, t_{2}\right)
\end{array}\right]
$$

Thereafter, utilization of equations (3.16) and (3.17) yields the $m$-th mode strength and phase.

The same procedure can be applied to data sets of the isopycnal displacements, with the only difference being the utilization of equation 3.9 over an incomplete domain rather than 3.10 .

\subsection{Regression method}

This section discusses the regression technique (Alford, 2003, Nash et al., 2005) utilized by much of the physical oceanographic community to determine the modal content of internal wave fields. In this technique, the horizontal baroclinic velocity and isopycnal displacements are expressed as:

$$
\begin{aligned}
& u(z, t)=\sum_{n=0}^{\infty} \tilde{\mathcal{U}}_{n}(t) \hat{u}_{n}(z), \\
& \rho(z, t)=\sum_{n=1}^{\infty} \tilde{\mathcal{H}}_{n}(t) \hat{\eta}_{n}(z),
\end{aligned}
$$

where $u(z, t)$ and $\eta(z, t)$ are the profiles determined from field data, and $\tilde{\mathcal{U}}_{n}(t)$ and 
$\tilde{\mathcal{H}}_{n}(t)$ are time-varying magnitudes of the baroclinic modes, to be determined. In this formulation, note that the time varying modal amplitude $\tilde{\mathcal{U}}_{n}(t)$ corresponds to the term $\Re\left\{\mathcal{U}_{n} e^{i\left(k_{n} z-\omega t\right)}\right\}$ from (3.12). The zeroth-mode $(n=0)$, corresponding to the barotropic solution, is also allowed in the case of the horizontal velocity.

The regression method is implemented as follows. For horizontal velocity measurements obtained at discrete vertical locations $z_{i}(i=1, M)$ in the water column, the data is configured as

$$
\left[\begin{array}{c}
u\left(z_{1}, t\right) \\
\vdots \\
u\left(z_{M}, t\right)
\end{array}\right]=\left[\begin{array}{ccc}
\hat{u}_{1}\left(z_{1}\right) & \ldots & \hat{u}_{n}\left(z_{1}\right) \\
\vdots & \ldots & \vdots \\
\hat{u}_{1}\left(z_{M}\right) & \ldots & \hat{u}_{n}\left(z_{M}\right)
\end{array}\right]\left[\begin{array}{c}
\tilde{\mathcal{U}}_{1}(t) \\
\vdots \\
\tilde{\mathcal{U}}_{n}(t)
\end{array}\right]
$$

and solved using standard overdetermined inverse methods (Dushaw et al. 1 1995) at each time for the unknown time-varying modal amplitudes, $\tilde{\mathcal{U}}_{n}(t)$; the same method is used for the time varying modal amplitudes of isopycnal displacement, $\tilde{\mathcal{H}}_{n}(t)$. Formally, a vertical resolution of $M$ data points enables the resolution of $M$ modes. In practice, the specific mooring geometry and the shape of the spectrum can further limit this value. In particular, Nash et al. (2005) notes that the regression method is especially sensitive to gaps in data near the surface. Furthermore, solving for more modes $M$ reduces the error, but affects the stability of the solutions.

\subsection{Results}

To test the effectiveness of both methods in determining the mode strengths of an internal wave field, an idealized horizontal wave field was generated in MATLAB using

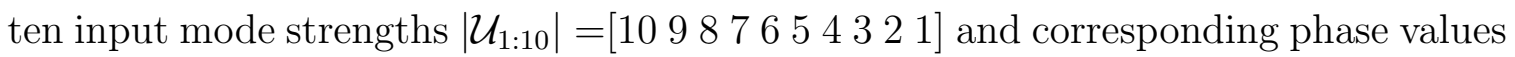
$\varphi_{1: 10}^{u}=\left[\begin{array}{llllllllllll}1 & 2 & 3 & 4 & 5 & 6 & 7 & 8 & 9 & 10\end{array}\right] \times \pi / 5$. The wave field was generated in a characteristic oceanic stratification shown in figure $3-2$ along with the corresponding first five modes of the horizontal velocity $\hat{u}(z)$ and isopycnal displacement $\hat{\eta}(z)$. The generated $u$ wave field is shown in figure 3-3. The corresponding isopycnal displacement field (not shown) was created using the same procedure as that used to construct the $u$ velocity, 
(a)

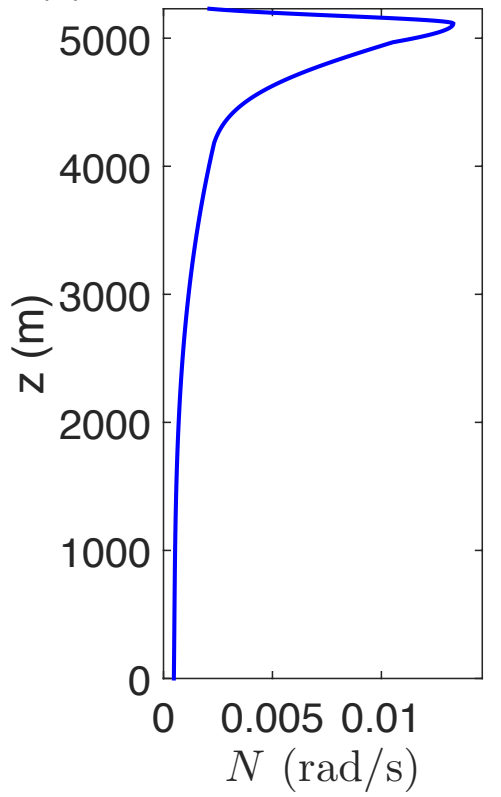

(b)

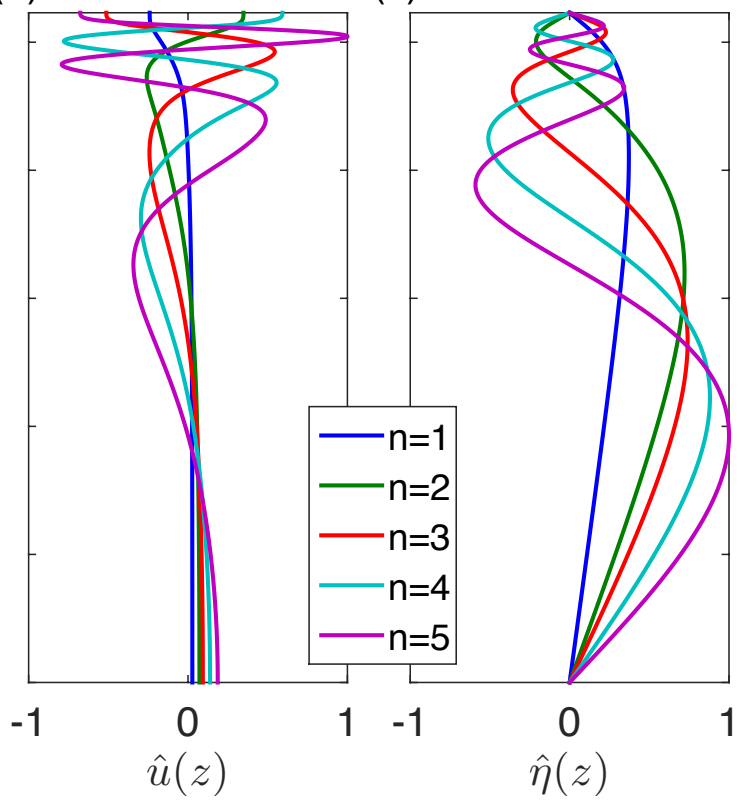

Figure 3-2: (a) Characteristic oceanic stratification used to generate wave field in figure 3-3 and corresponding vertical modes for the $(b)$ horizontal velocity, $\hat{u}(z)$, and $(c)$ isopycnal displacement, $\hat{\eta}(z)$ fields.

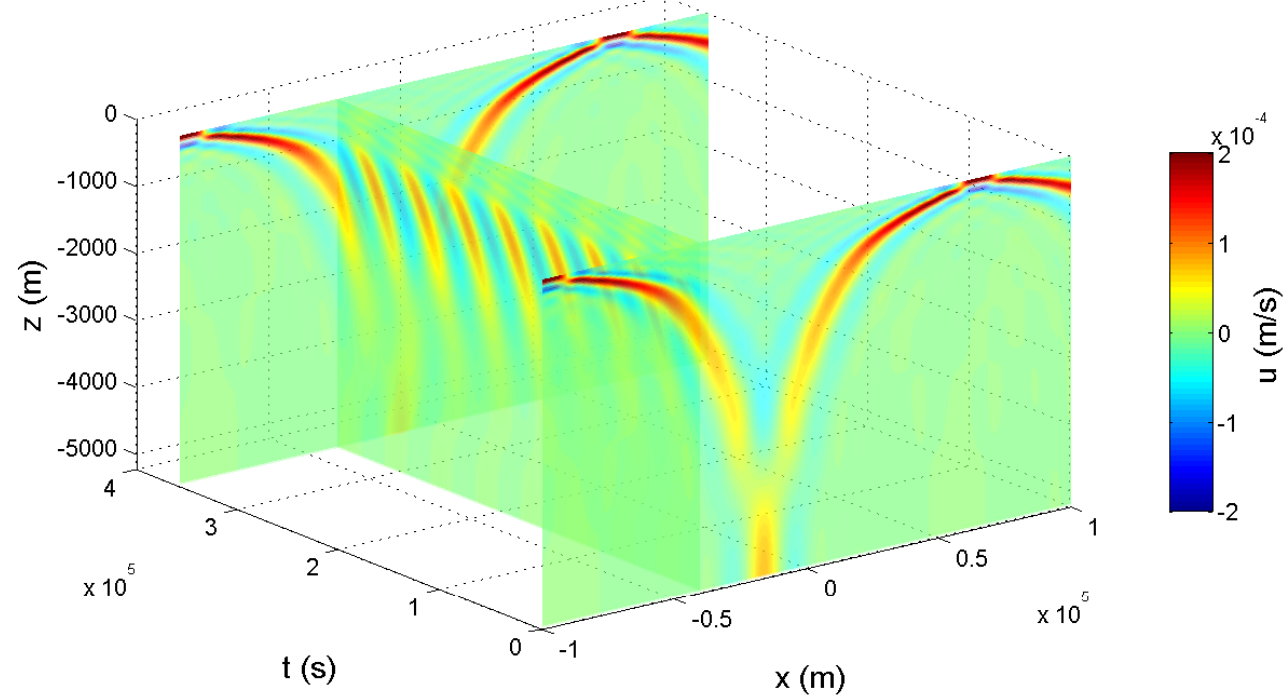

Figure 3-3: An idealized horizontal velocity field in a characteristic oceanic stratification plotted as a function of the spatial coordinates $x$ and $z$, and time $t$. The wave field was constructed in MATLAB using the known modal strengths $\left|\mathcal{U}_{1: 10}\right|=\left[\begin{array}{ll}10 & 98\end{array}\right.$

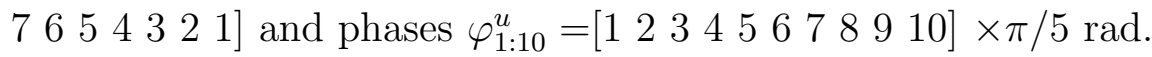

by virtue of using relations (3.19) and (3.20). 


\subsubsection{Full vertical domain}

Figure 3-4 presents the results of the projection method in determining the known modal amplitudes and phases for both $u$ and $\eta$, obtained using a time window of $\Delta t=\pi / 2 \omega$ and assuming full depth information. As one might hope, the agreement with the true values is excellent, with less than $1 \%$ error.

\subsubsection{Incomplete vertical domain}

The effectiveness in determining the modal composition of a wave field using the analytical and regression method was tested for varying cases of image loss from the top, bottom, and middle of the domain for both the $u$ velocity and $\eta$ displacement fields. In field studies, image loss at the top of the domain results from the fact that instruments cannot be placed on the mooring line to coincide with the exact free surface of the ocean. Furthermore, the placement of instrumentation throughout
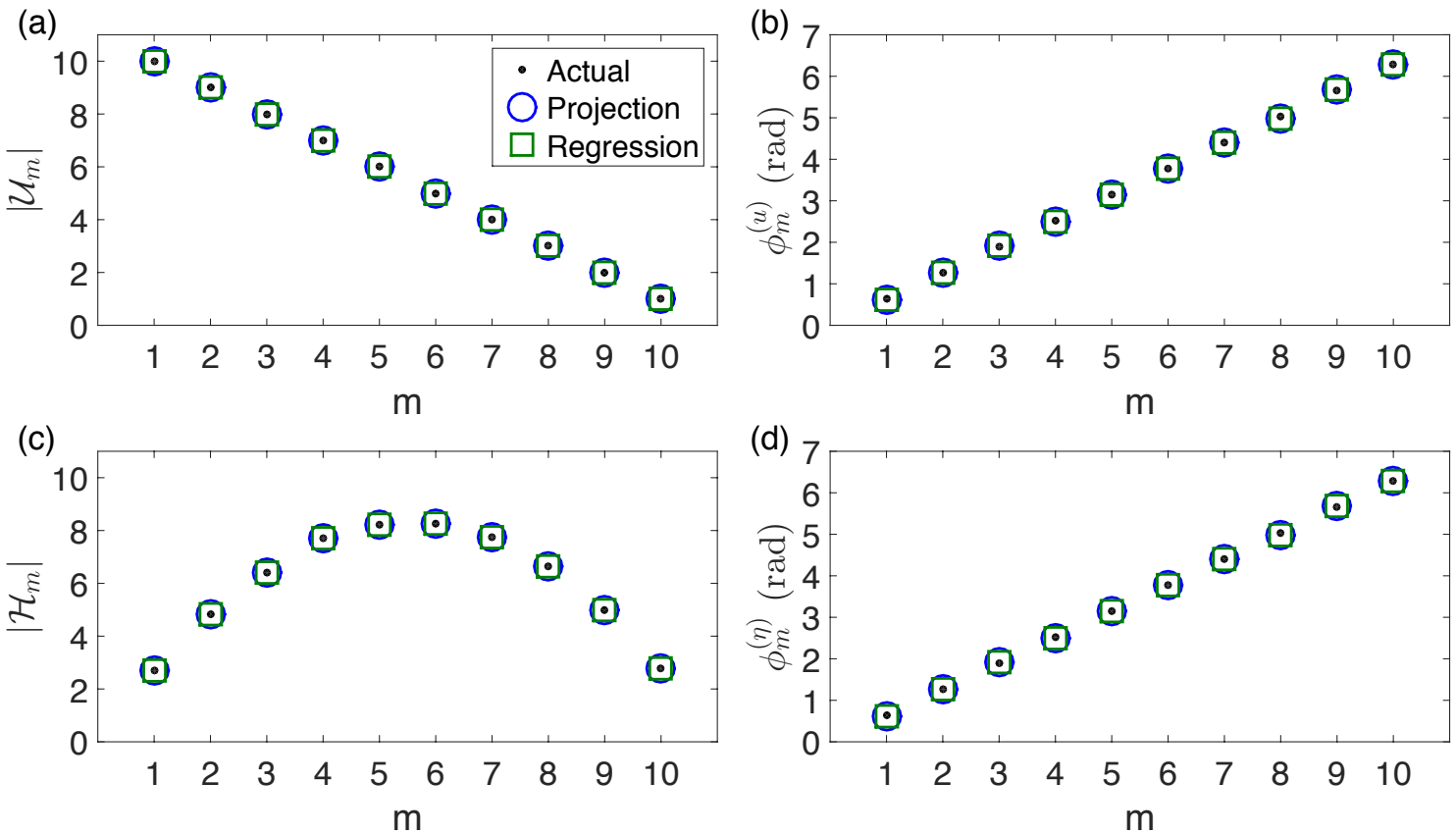

Figure 3-4: Modal decomposition results for the $u$ velocity profile (top row) and the isopycnal displacement $\eta$ (bottom row). Plots $(a)$ and $(c)$ display the modal strengths while $(b)$ and $(d)$ display the phases. The known inputs (black dots) are compared to the projection method results (blue circles) and regression method results (green squares). 
the water column will result in various additional regions of image loss in the middle of the ocean. Therefore, there is strong motivation to investigate the algorithm's performance in the presence of top, bottom, and middle image loss. The results of all these scenarios are presented in 3-5.

In the case of image loss, an unknown variable in determining the modal content of an internal wave field is the number of modes to be solved for $M$, in 3.24 . This variable is key in determining $\Upsilon \Upsilon$ and its inverse. As previously described, $\boldsymbol{\Upsilon}$ reduces to the identity matrix in the limit of full depth information for all $M$. Since the modal content is not known a priori, the maximum number of modes to decompose was set to be the highest resolvable mode by the velocity data $M=139$. The validity of using such a value of $M$ will be investigated in Section 3.6.

Figures 3-5(a) and 3-5(b) show the results of the percent error in the first five modes as a function of image loss from the top of the domain in the $u$ and $\eta$ fields respectively. The performance of the projection method is depicted by solid lines and that of the regression method by dashed lines. From the figure, it is clear to see that the projection method is able to sustain greater amounts of image loss than the regression method when accurately predicting the modal structure of the wave field for the $u$ velocity field, whereas regression out performs the projection method in the case of the $\eta$ displacement field. In the case of the $u$ velocity, figure $3-5(a)$ shows the projection method accurately predicts the modal amplitudes of the first five modes with less than $5 \%$ error for cases with less than 5\% image loss. The regression method, on the other hand, fails for all modes for cases of image loss of $3.3 \%$ or greater. Unlike the $u$ velocity field, the case is reversed for the $\eta$ displacement field, shown in figure $3-5(b)$. In this case, the projection method accurately predicts the first five modes within $5 \%$ for cases of image loss up to $4.3 \%$ while the regression method fails for cases with $7 \%$ image loss or more. The small amount of tolerance to image loss from the top of the vertical domain for both algorithms is due to the fact that the modal structures for the horizontal velocity and isopycnal displacements, as shown in figures $3-2(b)$ and $3-2(c)$, vary considerably within the first $10 \%$, or $523 \mathrm{~m}$ for the case of this idealized wave field, of the water column and thus this information 
(a)
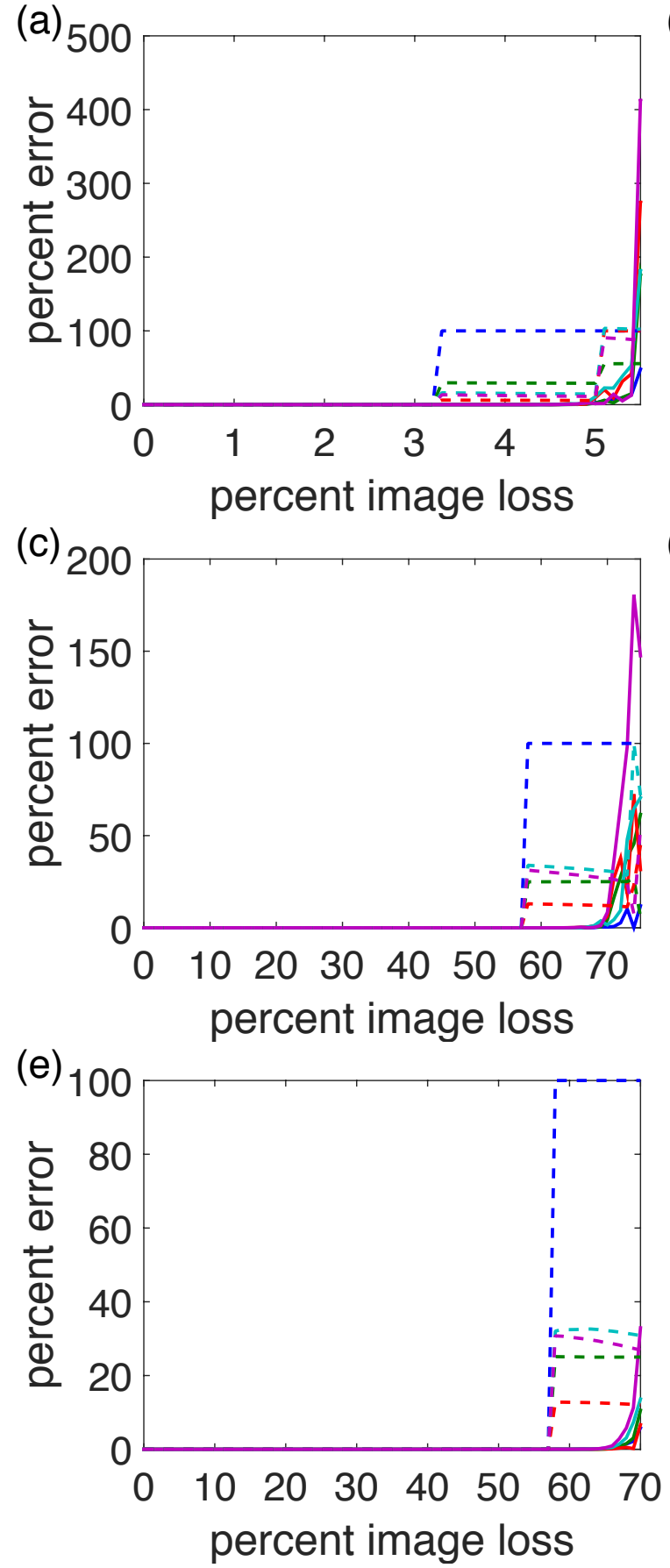

(b)

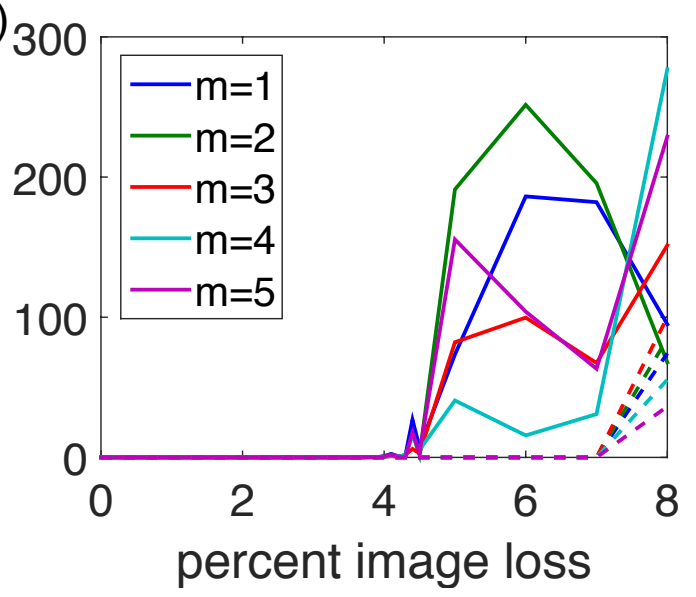

(d)

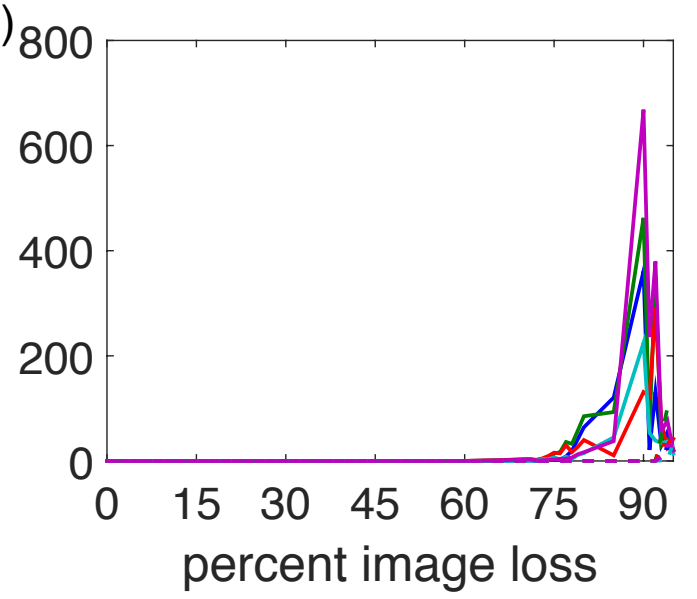

(f)

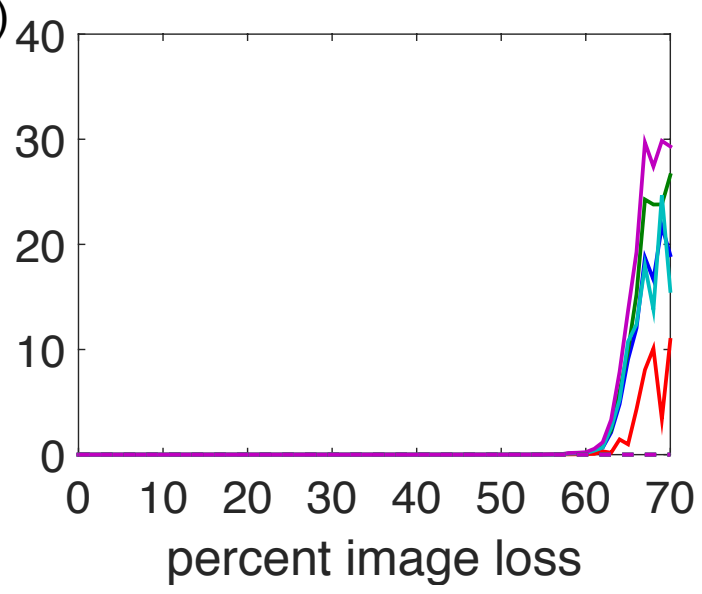

Figure 3-5: The percent error in the first five modes $m$ of the idealized wave field evaluated using the analytical procedure accounting for image loss (solid lines) and the regression method (dashed lines) as a function of image loss from the top of the vertical domain (top row), the bottom of the vertical domain (middle row), and the bottom of the vertical domain with combined image loss of $3 \%$ at the top and $2 \%$ in the middle (bottom row). Plots $(a),(c)$, and $(d)$ display the results for the $u$ velocity $(b),(d)$, and $(e)$ display the results for the $\eta$ displacement fields. 
is more valuable in distinguishing between the modes.

Although both algorithms show markedly little tolerance to image loss from the top, the projection method can handle up to $4.3 \%$ image loss whereas the regression method can only handle up to $3.3 \%$. This translates into roughly $225 \mathrm{~m}$ of image loss tolerable by the projection method as compared to $172 \mathrm{~m}$ for the regression method, which is significant difference of up to $53 \mathrm{~m}$. This is an extremely important result as the variability of mooring deployments near the surface in the deep ocean is generally on the order of $100 \mathrm{~m}$. Therefore, these results show that both methods are capable of distinguishing the modal content of an internal wave field using the $u$ velocity field for cases of image loss near the surface that are in keeping with current oceanographic limitations. However, as instrumentation for field studies change, the projection method has proven to be more robust for surface image loss allowing for an additional $53 \mathrm{~m}$ of uncertainty.

Similarly, image loss from the bottom of the domain is investigated in figure 3-5 (c) and $3-5(d)$ for both the $u$ velocity and $\eta$ displacement fields where the error levels of both the projection method and regression method are plotted against the percent of image loss. As can be seen in figure 3-5(c), in the case of the $u$ velocity field, the projection method can determine the first five modes within $5 \%$ for cases with up to $69 \%$ image loss. After this, the method can sustain up to $74 \%$ image loss and continue to predict the mode- 1 amplitude with less than $10 \%$ error. On the other hand, the regression method fails for cases with as little as $57 \%$ image loss, where, unlike the projection method, there is a large jump in the percent error for all modes. Figure 3-5( $(d)$ shows similar results for the case of the $\eta$ displacement field. Again, as in the case of image loss for the top, the case for the performance of the algorithms is reversed. The first five modes can be determined via the projection method within $5 \%$ error for cases with up to $73 \%$ image loss whereas the regression method does not fail for cases with up to $92 \%$ image loss. These results suggest that the regression method is much less sensitive to data loss in the $\eta$ displacement field, specifically from the bottom of the domain and into the pycnocline, than the projection method.

For the case of middle loss, the scenario of constant image loss at the top and 
middle of the domain in addition to varying amounts of image loss in the deep ocean was explored. An oceanographic data set with $3 \%$ image loss at the top, $2 \%$ image loss near the middle, and varying amounts of image loss in the deep ocean was used. Figure 3-5 (e) and 3-5( $f$ ) shows the results for the $u$ velocity field and $\eta$ displacement field, respectively, for both algorithms. In the case of the $u$ velocity field, the projection method results in less than $5 \%$ error in all five modes for up to $67 \%$ image loss at the bottom of the domain and can further predict the mode- 1 amplitude with less than $10 \%$ error for cases with up to $70 \%$ image loss at the bottom. On the other hand, the regression method fails for all five modes for cases of $58 \%$ bottom image loss or greater. Again, unlike the projection method, the change in error in the regression method as a function of increased image loss is sudden. Figure $3-5(f)$ shows the results for the $\eta$ displacement field. Here the projection method predicts the modal amplitudes for all five modes within 5\% for cases of image loss up to $60 \%$. Regression, however, is able to predict the modal amplitudes with less than $1 \%$ error for all five modes for all possibilities of bottom image loss. This is likely due to the fact that the analytical method is more sensitive to image loss at both the top and bottom of the domain for the $\eta$ displacement field than for the $u$ velocity field (see figure $3-5(a)$ and $3-5(b))$.

These results show that the projection method is more adept at handling image loss in the case of the $u$ velocity, whereas the regression method is less sensitive to image loss in the $\eta$ displacement field. Given that the quantities of most interest to oceanographic studies are the energy and energy flux of an internal tide, variables that are determined from a combination of measurements of $u$ and $\eta$ displacement, these results suggest that the projection method can determine these quantities in instances of greater image loss than the regression method, thereby making it a technique to be considered for use in oceanographic field studies. 


\subsection{Ocean example}

To verify the algorithm presented in 3.2 , a synthetic data set representative of that gathered from oceanographic field experiments is generated. Green function theory utilized in Chapter 2 is again used to produce the modal content of a wave field based on a two-dimensional topography and then to further generate a synthetic wave field from these modal amplitudes and phases.

Given that the goal of this chapter is to determine the best modal decomposition technique to be utilized to analyze field observations from the EXITS study, the modal decomposition of an internal wave field generated by the $M_{2}$ tide $\left(\omega=1.4053 \times 10^{-4}\right)$ at the Line Islands Ridge was investigated. Specifically, the northward propagating generated internal tide at the N-S transect of the Ridge (figure 2-10) was studied. The stratification at and the topography of this transect is shown in figure 2-12.

It is widely understood that high mode internal tides dissipate locally due to interactions with small-scale features. Hence, it is highly unlikely that modes higher than 30 will be present in an oceanographic wave field. With this in mind, the Green function method was used to determine the generated internal wave field at

(a)

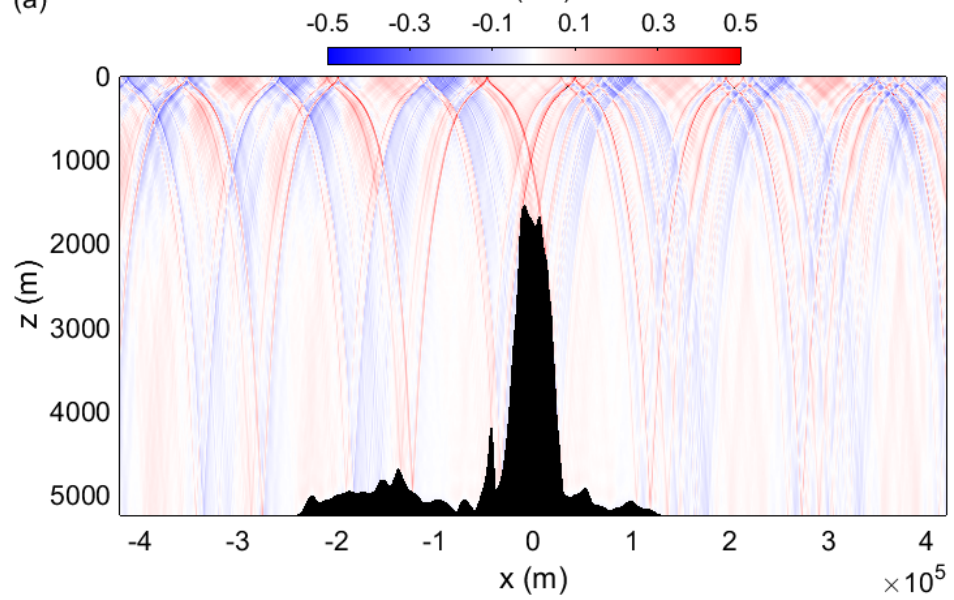

(b)

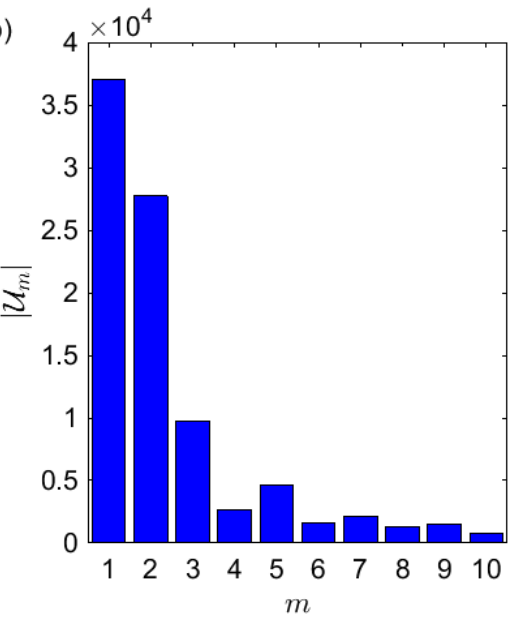

Figure 3-6: The (a) topography of the Line Islands Ridge transect investigated as well as the northward (leftward) and southward (rightward) propagating horizontal velocity field $(u(x, z, t))$ at an arbitrary instant in time of the generated internal tide. In $(b)$, the modal amplitude of the first ten modes of the northward propagating internal tide as determine by the Green function approach. 
the N-S transect of the EXITS study with 30 modes. The topography of the transect investigated as well as the generated internal wave field as predicted by the Green function approach is show in figure $3-6(a)$. Figure $3-6(b)$ shows the modal amplitudes of the first ten modes for the northward propagating internal tide indicated in figure 36(a) as determined by the Green function approach. It is clear from 3-6(b) that the northward propagating internal tide is mode- 1 dominated. In order to investigate the performance of the analytical method in an oceanographic setting representative of the EXITS study, image loss consistent with mooring $U$ was implemented on the northward propagating internal wave field depicted in figure 3-6 (a). This resulted in a wave field with $100 \mathrm{~m}$ of loss at the top $(\sim 2 \%), 100 \mathrm{~m}$ of loss at the middle from $1450 \mathrm{~m}$ to $1550 \mathrm{~m}(\sim 2 \%)$, and $2390 \mathrm{~m}$ of data loss at the bottom $(\sim 45.7 \%)$.

Again, as in the investigation of incomplete vertical domain data in section 3.4 .2 , since the modal content is not known a priori, the maximum number of modes to decompose was set to be the highest resolvable mode, $M=139$. The results shown in

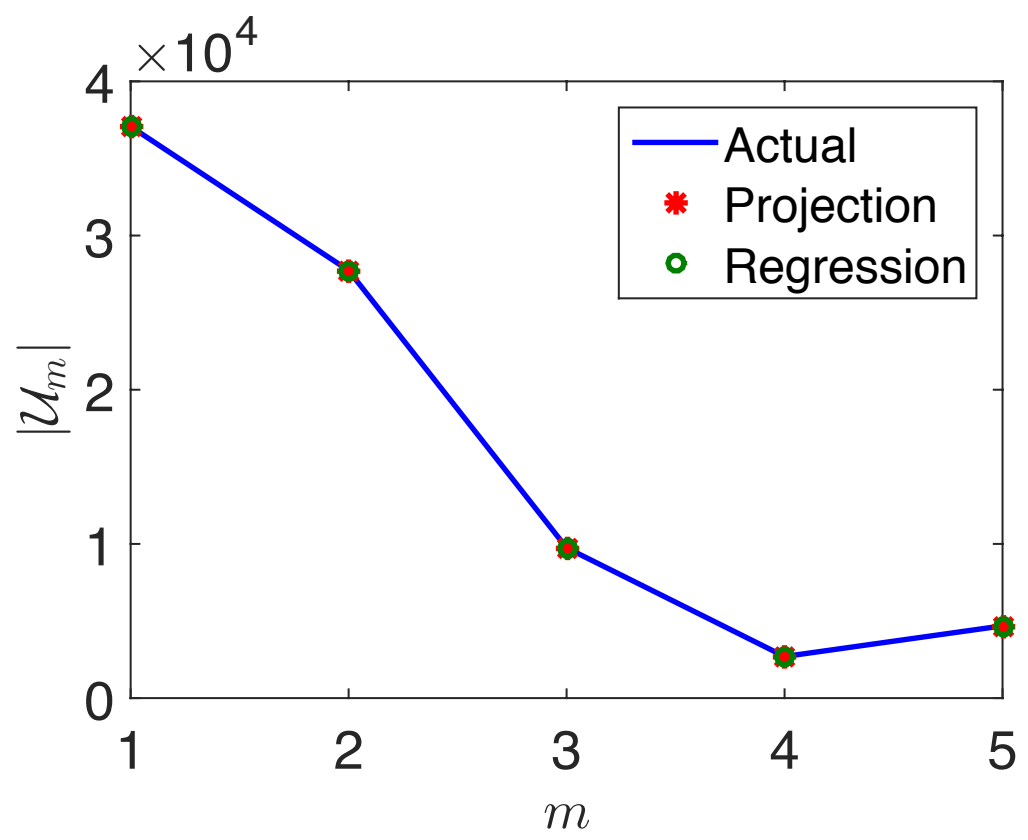

Figure 3-7: The modal amplitudes for the first five modes of the generated $M_{2}$ internal tide at the N-S transect of the Line Islands Ridge as determined by the Green function theory (blue line), projection method (red stars), and regression method (green circles). 
figure 3-7 indicate that even in such extreme cases of image loss, both the projection method and regression method perform very well. The error in the prediction of the modal amplitudes is $<1 \%$ for modes $1-3$ for both methods.

These results suggest that in the case of the $u$ velocity field, both methods are quite adept at handling oceanic cases of image loss. The results from this section show that even in the case of extreme image loss seen at mooring U of the EXITS study, both the projection and regression methods should be able to accurately predict the amplitudes of the first five modes. Therefore, in keeping with current oceanographic studies, the regression method will be used to determine the modal content of the internal tide at each of the three moorings, as described in Chapter 4.

\subsection{Determining $M$}

As described previously, when there is full depth information, the projection method independently determines each modal amplitude since $\Upsilon$ is an identity matrix, and so one can determine modal content up to whatever mode is desired. When there is incomplete vertical data, however, then one must choose a value for $M$ from (3.24) corresponding to the highest mode present in the data as the solution procedure is coupled; this is also an issue for the regression method.

This issue is first examined using the ideal wave field from Section 3.4 with a reasonable oceanic scenario with data loss in the upper 3\% and lower $60 \%$ of the water column. As shown in figure 3-8, when varying $M$ used in both methods there is significant amount of variability in the computed amplitudes of the first three modes. Specifically, both the projection method and the regression method have large errors when using $M<10$, the number of modes present in the wave field. This suggests that even though modes 1-3 of an internal wave are generally the only modes of interest, the number of modes solved for $M$ has an effect on the amplitudes of all modes computed. Secondly, while the projection method performs extremely well, predicting the amplitudes with less than $1 \%$ error for the modal amplitudes of modes 1-3, for $M>65$, the regression method fails. 


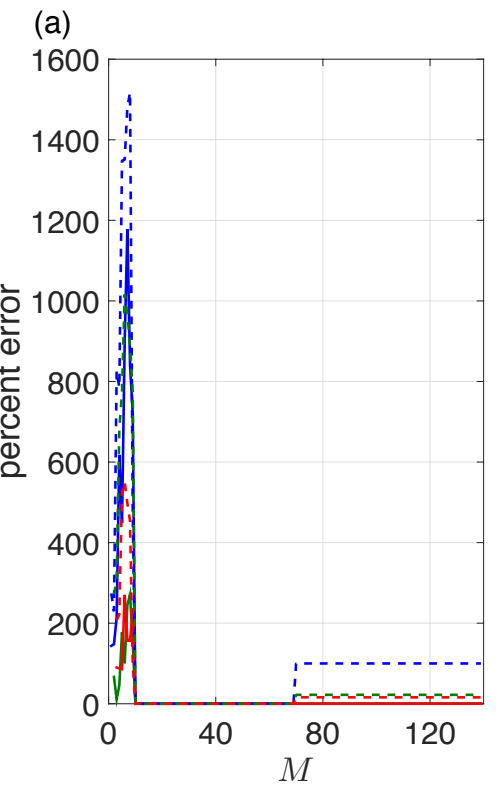

(b)

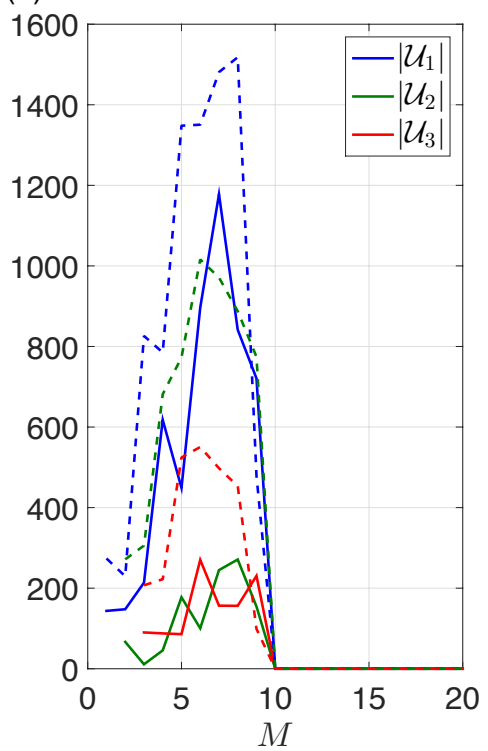

(c)

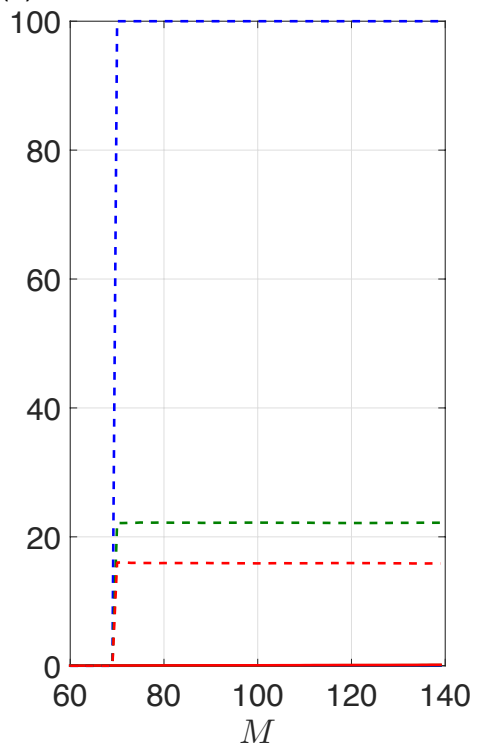

Figure 3-8: The percent error in computing the amplitudes of mode 1 (blue), mode 2 (green), and mode 3 (red) with varying $M$ when using the projection method (solid lines) and the regression method (dashed lines) for the idealized wave field presented in Section 3.4 with $3 \%$ top image loss and $60 \%$ bottom image loss. In (a) $M$ ranges 1 to 139 . Zoomed-in plots of the percent error for $M$ ranging from (b) 1 to 20 and (c) 60 to 139.

Similarly, the effect of varying $M$ for a realistic oceanic wave field was investigated using the internal wave field from Section 3.5, the results of which are shown in figure 3-9. Here the image loss is again set to be that of mooring $U$ from the EXITS cruise. As in figure 3-8, there is a large variability in the computed modal amplitudes when varying $M$ used in both methods. Both methods again have large errors when $M$ is less than the number of modes present in the wave field, $M<30$, after which both methods predict the modal amplitude with less than $1 \%$ error. However, unlike the idealized wave field, the regression method does not diverge for any large value of $M$. Additionally, while the peak error occurred around $M=8$ in figure $3-8$ for the idealized wave field, the error peaks for the oceanic wave field when $M \sim 26$.

These findings suggest that further work must be done to determine the value of $M$ that should be utilized with the projection method to ensure the algorithm converges. Since the number of modes in a wave field is unknown, $M$ will likely relate to the vertical resolution of the data as well as the locations of the regions of image loss 

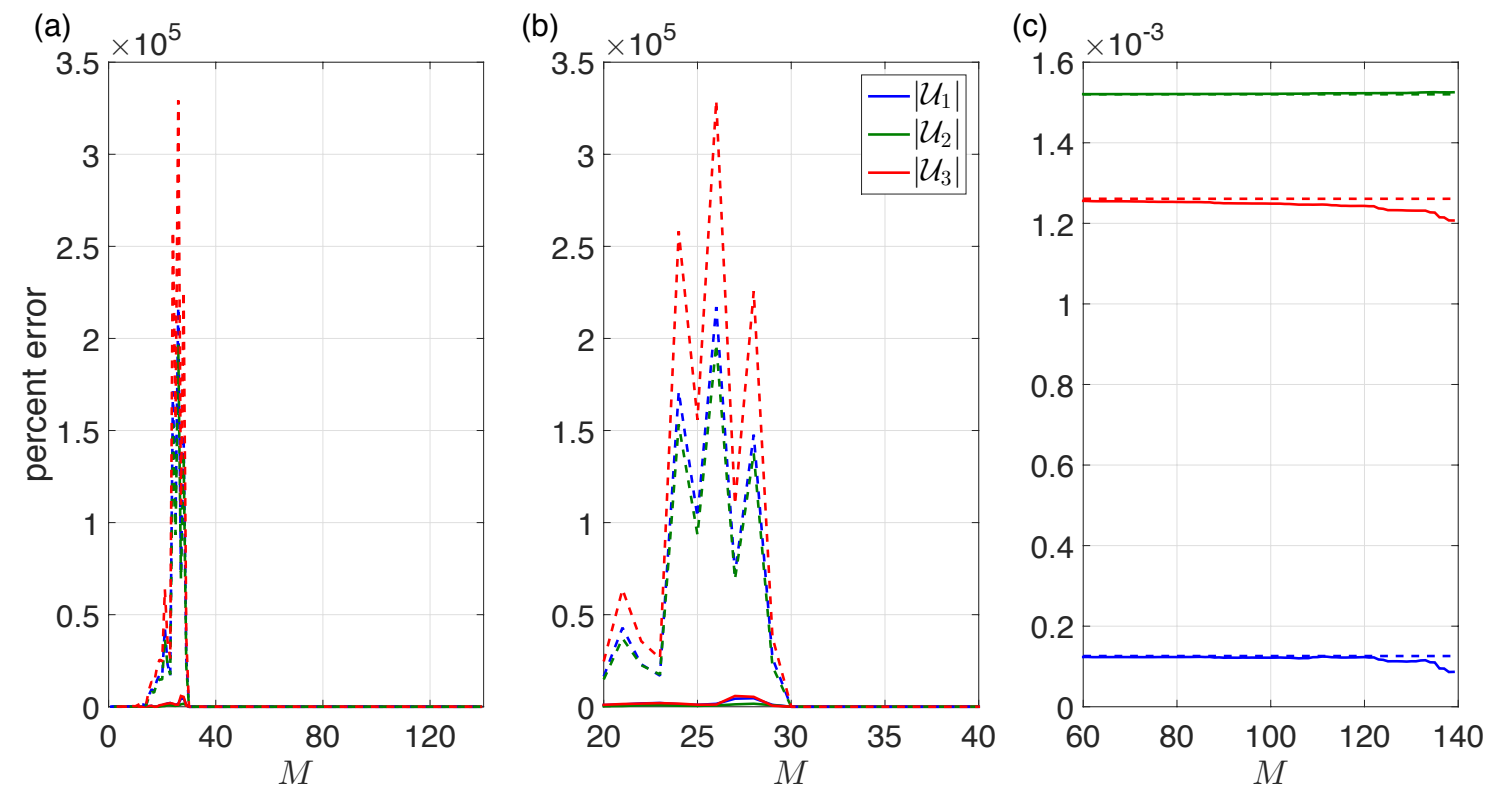

Figure 3-9: The percent error in computing the amplitudes of mode 1 (blue), mode 2 (green), and mode 3 (red) with varying $M$ when using the projection method (solid lines) and the regression method (dashed lines) for the idealized wave field presented in Section 3.5 with image loss consistent with mooring $U$ from the EXITS cruise. In (a) $M$ ranges 1 to 139. Zoomed-in plots of the percent error for $M$ ranging from $(b)$ 20 to 40 and (c) 60 to 139.

and their extent. Additionally, choosing $M$ also greatly affects the currently widely used regression method, therefore its performance must also be investigated for the determination of the optimum modes to be solved for. At present, oceanographers simulate data sets using Monte Carlo methods (as described by Nash et al. (2005)) of synthetically generated wave fields made up of the superimposition of 30 modes. Data is then removed at regions of image loss consistent with the mooring geometry in an oceanographic experiment and the regression method is run to determine the modal content using various values for $M$. The value of $M$ chosen is the one for which the modal amplitudes determined from the full-depth and partial-depth synthetic data are in agreement. In general, $M=5,10$ is often used based on these analyses (Zhao et al., 2010). 


\section{Chapter 4}

\section{Field Experiment Results}

\subsection{Introduction}

In this chapter, the results from the EXperiment on Internal Tide Scattering (EXITS) oceanographic field study are discussed. Conducted from November 2010 to May 2011, the EXITS cruise was the first field study to examine the internal tide scattering process in the ocean. It was motivated by observations that at the Line Islands Ridge there is a rapid decay of the large, southwest propagating mode- 1 baroclinic energy flux generated at French Frigate Shoals (Ray \& Cartwright, 2001), coinciding with an abrupt change in wave number and amplitude of the internal tide surface elevations (Ray \& Mitchum, 1997, Johnston et al., 2003). Heading north from the Hawaiian ridge system, however, Zhao et al. (2012) show that low-mode internal tides propagate over $3500 \mathrm{~km}$ across the generally small-scale, rough topography of the North Pacific Ocean. Furthermore, as mentioned in Chapter 1, Johnston et al. (2003) found that the altimetric sea surface height modulations due to the mode-1 $M_{2}$ internal tide propagating southward from the Hawaiian Ridge weaken at a prominent seamount in the Line Islands Ridge, where modulations due to the mode- $2 M_{2}$ internal tide increase, a clear indication of internal tide scattering. Additionally, numerical approaches have indicated that $19 \%$ of the incident energy from a mode- 1 internal tide generated at the Hawaiian Ridge is scattered at Line Islands Ridge into modes 2-5 (Johnston et al., 2003). 
This chapter begins by describing the measurement instruments used in the EXITS study as well as the methods to analyze their data in Section 4.2. Section 4.3 details the describes the results of the measurement analysis, and Section 4.4 discusses the uncertainties with the analysis. Finally, Section 4.5 describes the major conclusions of this study.

\subsection{Data and methods}

From the data collected using the moored profilers, we seek to identify the modal content of the semidiurnal internal tide incident and downstream of the Line Islands Ridge. This is done by first isolating the semidiurnal internal tide using bandpass filtering. The bandpassed velocity and isopycnal displacements are then decomposed into the five lowest baroclinic modes. From this, the quantities of pressure, energy, and energy flux in each of the modes can be computed. This section describes the EXITS field study, as well as the methods used to analyze the moored profiler data.

\subsubsection{The EXITS field study}

The EXperiment on Internal Tide Scattering (EXITS) was designed to examine the southwestward mode-1 internal tide beam originating from French Frigate Shoals, Hawaii scatter into higher mode waves at the Line Islands Ridge. Observations from Topex/Poseidon (Zhao \& Alford, 2009) and numerical modeling using the Princeton Ocean Model (POM) (Johnston \& Merrifield, 2003) show mode-1 internal tides are diminished at the Line Islands Ridge, while mode-2 internal tides emanate southward from the ridge. The EXITS field cruise focused on the scattering of internal tides generated at the Hawaiian Ridge, complementing the Hawaii Ocean Mixing Experiment (HOME) which focused on the internal tide's generation and local dissipation at the Hawaiian Ridge.

Figure 4-1 details the EXITS region of study. To observe this scattering, three

moorings (stars, detailed in Table 4.1) were deployed and 18 stations (dots) were occupied for 12-36 hours each with CTD, lowered ADCP (LADCP), and untethered 


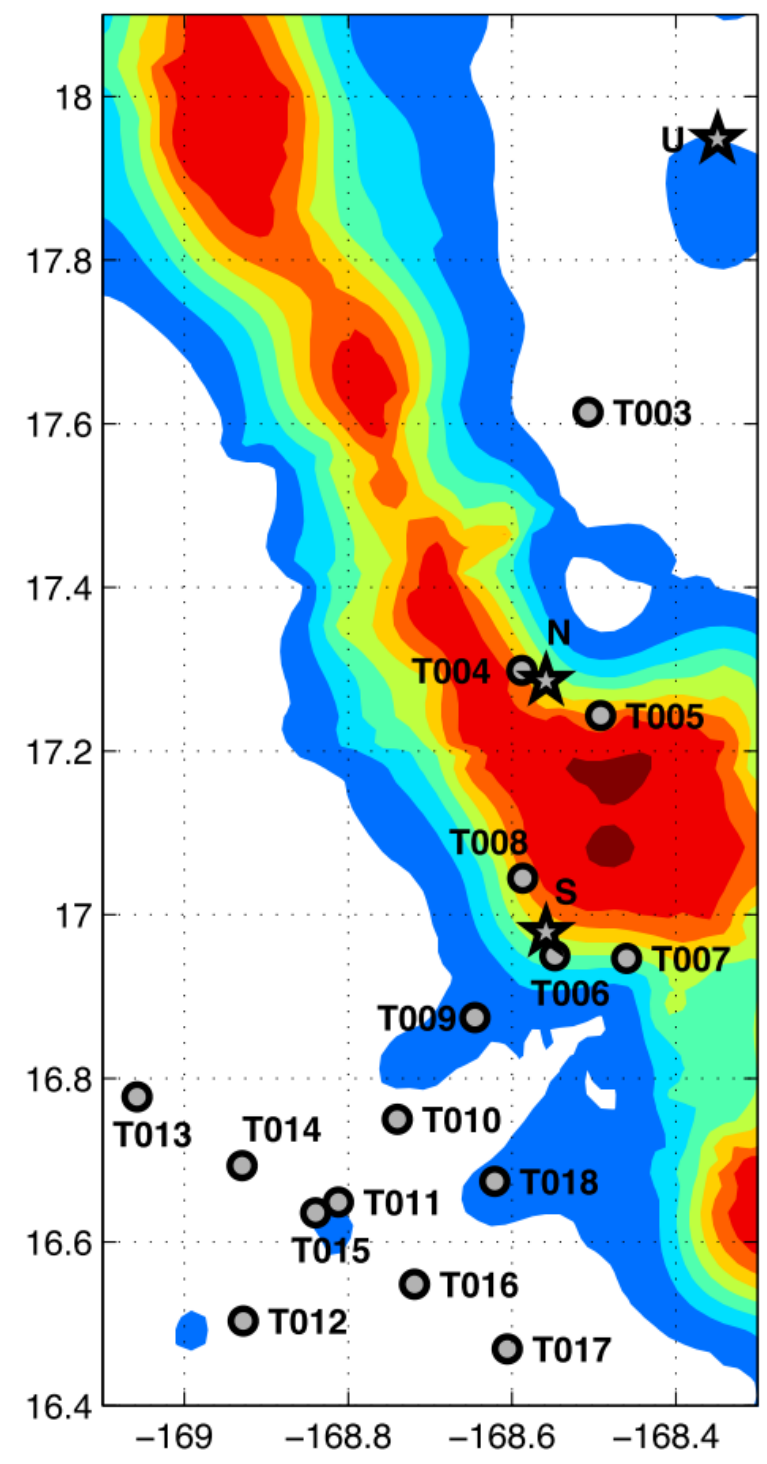

Figure 4-1: CTD/LADCP/microstructure stations were occupied from 12-36 hours and are labelled in order of occupation (dots). T011 and T015 are actually the same location but occupied at different times and slightly offset in this and following figures for clarity. Mooring U, N, and S locations are noted (stars). Colored contours indicate topography at 500-m intervals starting at $1500 \mathrm{~m}$.

microstructure stations. Only the moored data are discussed in this thesis. Moored measurements were conducted spanning mooring $\mathrm{U}$ at $17.9^{\circ} \mathrm{N}$ and $168.4^{\circ} \mathrm{W}$, about $100 \mathrm{~km}$ upstream of the Line Islands Ridge (with respect to the incident mode-1 internal tide), and mooring $\mathrm{S}$ at $16.9^{\circ} \mathrm{N}$ and $168.6^{\circ} \mathrm{W}$, about $5 \mathrm{~km}$ downstream of the Line Islands Ridge. With respect to the respect to the incident mode-1 wave, mooring $\mathrm{U}$ is upstream of the Line Islands Ridge in $\sim 5000 \mathrm{~m}$ of water. Both mooring $\mathrm{N}$ on 
Table 4.1: EXITS profiling moorings

\begin{tabular}{ccc}
\hline \hline Mooring & Location $\left({ }^{\circ} \mathrm{N},{ }^{\circ} \mathrm{W}\right)$ & Depth $(\mathrm{m})$ \\
\hline $\mathrm{U}$ & $17.9,168.4$ & 5094 \\
$\mathrm{~N}$ & $17.3,168.6$ & 3177 \\
$\mathrm{~S}$ & $16.9,168.6$ & 3073 \\
\hline
\end{tabular}

the north (upstream) side of the ridge and mooring $\mathrm{S}$ on the south (downstream) side of the ridge are in $\sim 3000 \mathrm{~m}$ of water. Mooring $\mathrm{U}$ was designed to observe the incident mode-1 wave before it encounters the Line Islands Ridge. According to model results, most of the scattering takes place on the downstream side of the ridge (Johnston \& Merrifield, 2003). Mooring N was deployed where the incident mode-1 wave was expected to first encounter the ridge, and mooring $\mathrm{S}$ was sited in the region of strongest expected conversion from mode 1 to higher modes.

The experiment consisted of three cruises: the first was from 23 November to 22 December 2010 aboard the R/V Thompson G. Thompson, the second and third were aboard the R/V Kilo Moana from 14 to 26 January 2011 and 26 April to 14 May 2011 respectively. All moorings were deployed twice: first in November 2010 for $\sim 50$ days, recovered and turned around in January 2011 and then deployed again for an additional $\sim 100$ days, after which they were recovered in April/May 2011.

\subsubsection{Profiling mooring instruments}

Each mooring was equipped with two McLane moored profilers (MMP) covering the upper $3000 \mathrm{~m}$, each climbing up and down through the water column along the mooring wiring, completing one up or down profile every $\sim 1.5 \mathrm{~h}$. Each carried a Falmouth Scientific CTD and acoustic current meter, delivering profiles of density and velocity with a vertical resolution of about $2 \mathrm{~m}$ (Doherty et al., 1999). All MMPs were programmed to profile continuously for the first deployment and continuously for 14 days every 14 days during the second deployment. Single point current meters (CM) measuring the velocity at $2 \mathrm{MHz}$ were mounted near the top and bottom of each mooring, with the exception of the second deployment of mooring $\mathrm{S}$ in which the CM was mounted at mid-depth. Additionally, each mooring was equipped with a 
(a)

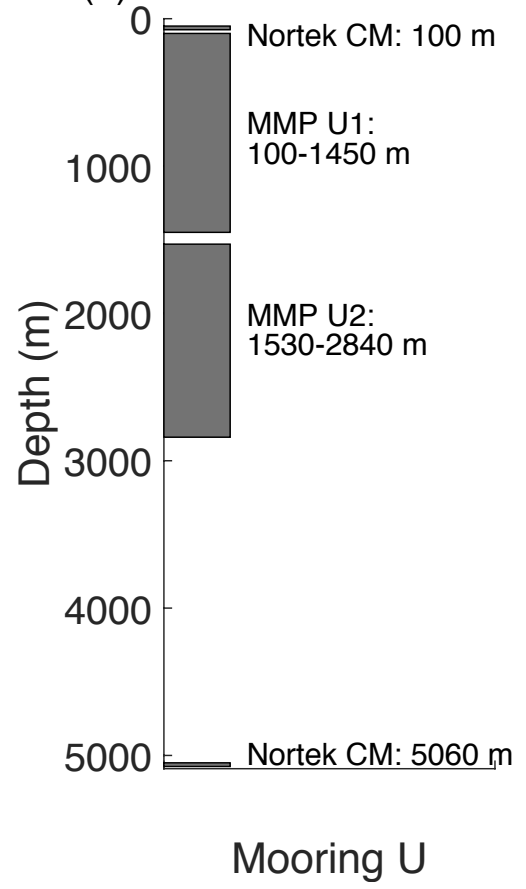

(b)

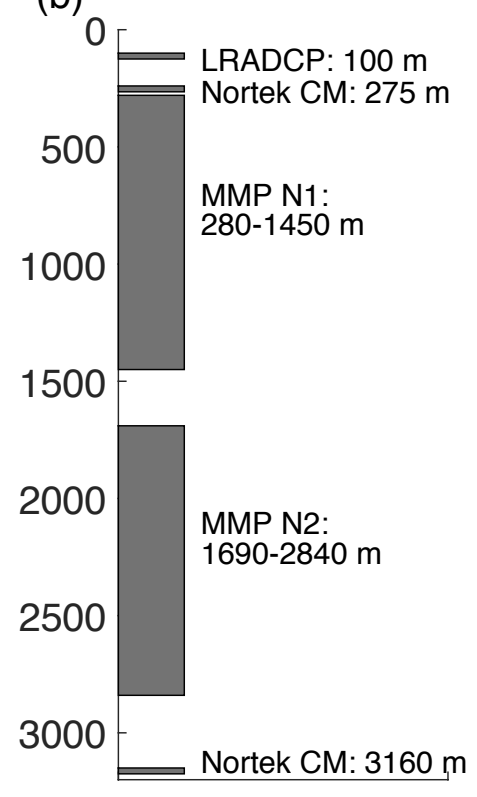

Mooring $\mathrm{N}$ (c)

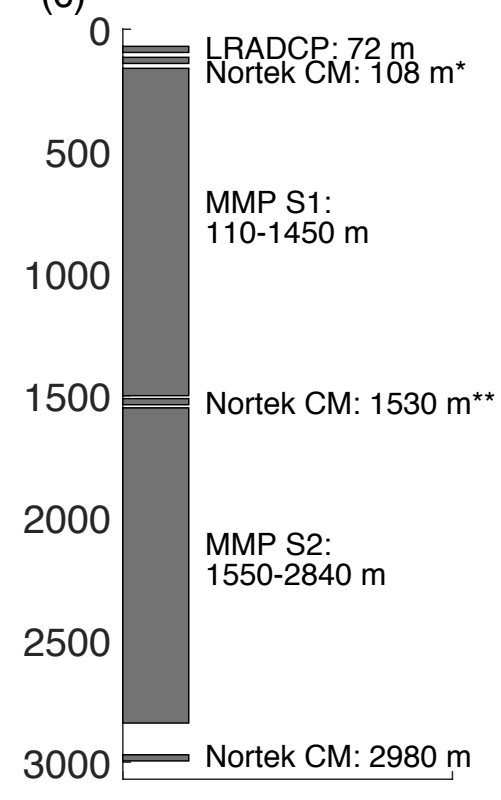

Mooring S

Figure 4-2: Instrumentation of moorings used in EXITS cruise and their average measurement locations at $(a)$ mooring $\mathrm{U},(b)$ mooring $\mathrm{N}$ and $(c)$ mooring $\mathrm{S}$. Note that only instruments measuring velocity are shown. In the case of mooring $\mathrm{S}, *$ indicates instruments only present during the first deployment and ${ }^{* *}$ indicates instruments only present during the second deployment.

microcat CTD at mid-depth. Moorings $\mathrm{N}$ and $\mathrm{S}$ also had downward-looking $75 \mathrm{kHz}$ Longranger ADCPs (LRADCP) between 70 and $100 \mathrm{~m}$. Details of each mooring are show in figure $4-2$.

The instruments generally functioned well. On mooring $\mathrm{U}$, both top and bottom MMPs sampled for the full duration of the first deployment, however the bottom MMP stopped sampling the entire lower vertical domain for the last $\sim 12$ days of the second deployment. On mooring N, the top MMP failed after the first 28 days of the first deployment and was replaced for the second deployment. On mooring $\mathrm{S}$, the top MMP failed for the entirety of the first deployment and was replaced for the second deployment.

The measured velocity field is decomposed into north-south $(v)$ and east-west $(u)$ components. The left panel of figure $4-3$ shows samples of the raw data at moorings $U$, $\mathrm{N}$, and $\mathrm{S}$ over the second deployment where colors indicate the north-south velocities 
obtained from the MMPs for each mooring. The measured velocities indicate a variety of low-frequency and near-inertial flows in the region. Bandpass filtering, as described below, was therefore utilized to isolate the tidal signals from the velocity data.

The MMPs trace a sawtooth pattern when profiling which leads to a variable temporal resolution ranging from $3 \mathrm{~h}$ at the top and bottom of their profiling ranges and $1.5 \mathrm{~h}$ at mid-depth. All data were gridded onto a uniform 1.5-h temporal and 2-m vertical resolution using linear interpolation.

\subsubsection{Bandpass filtering}

Bandpass filtering was used to isolate the semidiurnal components of the mooring data. A passband was chosen that includes the $M_{2}$ and $S_{2}$ tidal constituents but rejects inertial motion, whose frequency ranges between 0.99 and $1.39 \times 10^{-5} \mathrm{~s}^{-1}$ in this latitude range. This was done by bandpassing the data at each depth using a fourth-order Butterworth filter centered at the $M_{2}$ tidal frequency $\left(2.23 \times 10^{-5} \mathrm{~s}^{-1}\right)$ with zero-phase response and quarter-power points at $\{2.01,2.47\} \times 10^{-5} \mathrm{~s}^{-1}$. As described in Section 4.2.5, the barotropic currents were also removed.

The resulting baroclinic semidiurnal signals are plotted in the right panels of figure 4-3 in which currents with amplitudes $O(10 \mathrm{~cm} / \mathrm{s})$ can be clearly seen. Section 4.3 details the implications of these signals further.

\subsubsection{Vertical displacement}

For the top $\sim 3000 \mathrm{~m}$ of the water column, the vertical displacement was calculated from isopycnal displacements using linear interpolation of the density profiles as measured from the top and bottom MMPs of each mooring. A 3-day sliding window was used to remove signals associated with long-term density changes (such as due to passing eddies). Raw and semidiurnally bandpassed vertical displacements are also shown in figure $4-3$. 


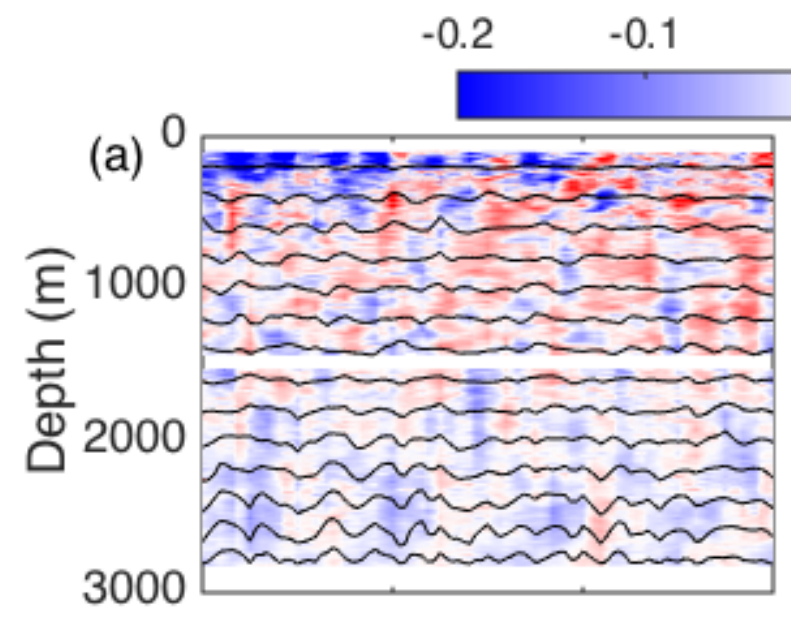

$\begin{array}{lll}0 & 0.1 & 0.2\end{array}$

(b)
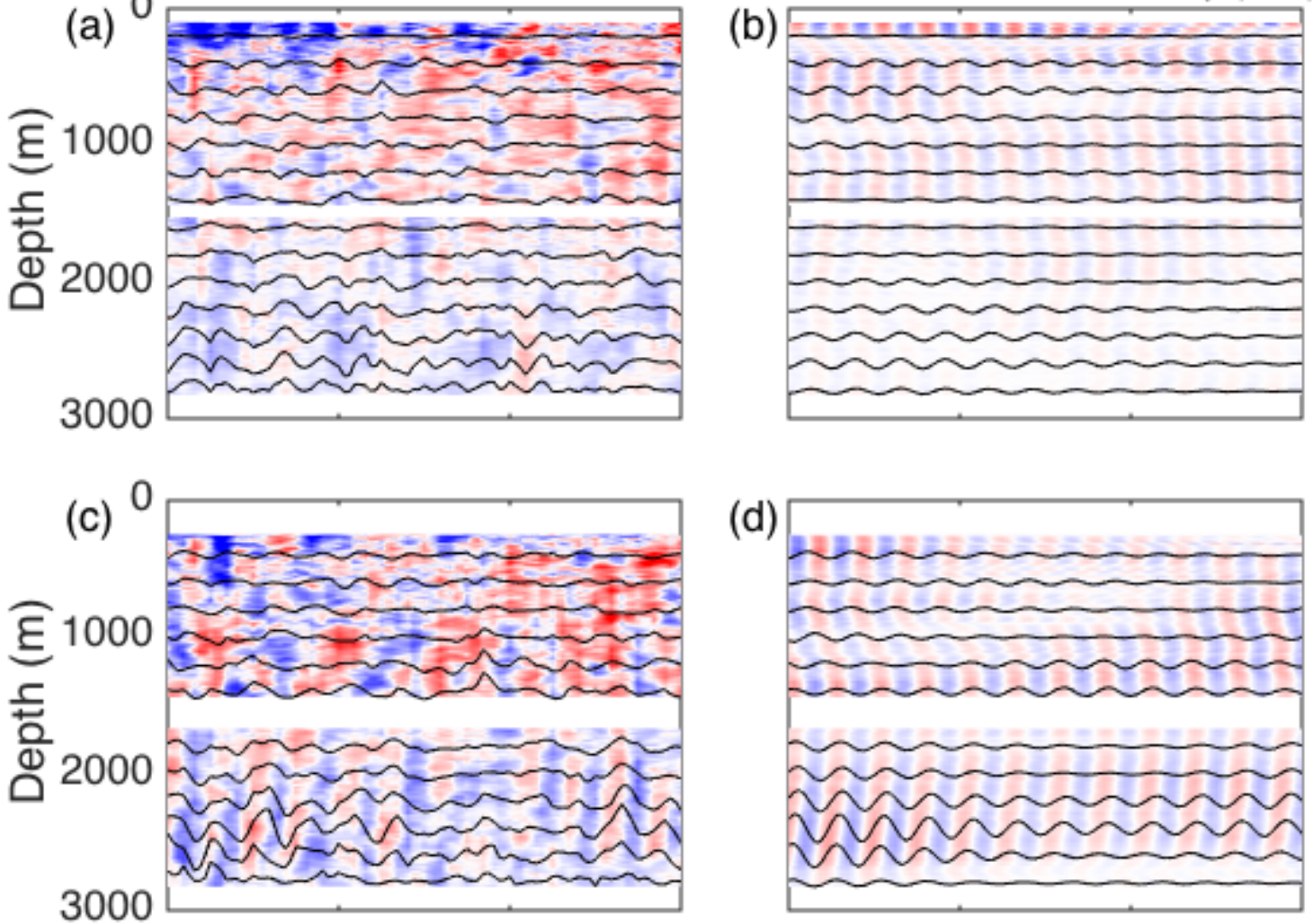

(d)
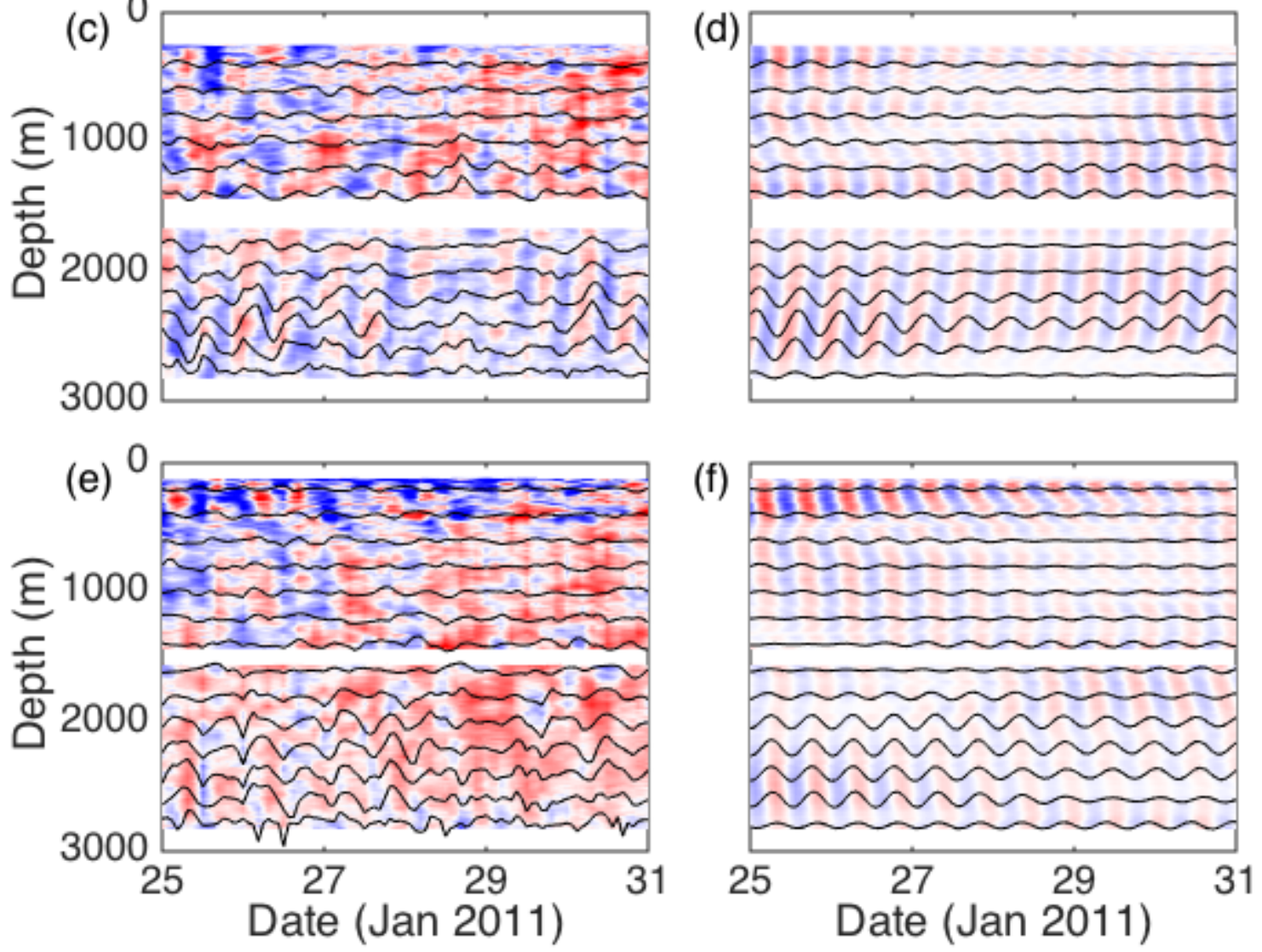

Figure 4-3: Samples of (left) raw measurements and (right) bandpassed semidiurnal signals at $(a),(b)$ mooring $\mathrm{U},(c),(d)$, mooring $\mathrm{N}$, and $(e),(f)$ mooring $\mathrm{S}$. Isopycnal displacements with mean spacing of $100 \mathrm{~m}$ are plotted in black. 


\subsubsection{Modal decomposition}

Determining the modal content of the internal wave field before and after encountering the Line Islands Ridge is the main goal of the EXITS study. In order to do this, the semidiurnal bandpassed signals are decomposed into baroclinic modes. Over an ocean of constant depth, the internal tides' vertical structure can be represented by a superposition of discrete vertical baroclinic modes that depend only on the buoyancy frequency profile, $N(z)$. Following the work of oceanographic studies by Alford (2003), Alford \& Zhao (2007) and Zhao et al. (2010), the baroclinic modes for the vertical structure of the internal tide, $\Phi(z)$, can be determined by the eigenvalue equation,

$$
\frac{d^{2} \Phi(z)}{d z^{2}}+\frac{N^{2}(z)}{c_{n}^{2}} \Phi_{n}(z)=0
$$

subject to the boundary conditions $\Phi_{n}(0)=\Phi_{n}(H)=0$, where $H$ is the water depth, $n$ is the mode number, and $c_{n}$ is the eigenspeed (Gill, 1982). $N(z)$ is determined from shipboard CTD measurements taken near each of the moorings during the EXITS cruises. The shipboard stratification profile (shown for mooring $\mathrm{N}$ in figure 4-4 (a), blue line) is consistent with the MMP measured stratification profile (shown for mooring $\mathrm{N}$ in figure 4-4(a), dashed green line). The stratification and mode shapes were similar at all three mooring locations.

The baroclinic modes of the vertical displacement are given by to $\Phi_{n}(z)$, while the baroclinic modes of horizontal velocity and pressure are linearly proportional to $\Pi_{n}(z)$ which is defined as

$$
\Pi_{n}(z)=\rho_{0} c_{n}^{2} \frac{d \Phi_{n}(z)}{d z}
$$

where $\rho_{0}$ is the water density. The normalized baroclinic modes structures at mooring $\mathrm{N}$ for the horizontal velocity and pressure, $\Pi_{n}(z)$, and the vertical displacement, $\Phi_{n}(z)$, are depicted in figures 4-4(b) and 4-4(c), respectively.

Given the dispersion relation, for each mode, the eigenspeed $\left(c_{n}\right)$ can be used to derive the phase velocity, $c_{p, n}$, and group velocity, $c_{g, n}$, along the direction of propagation as 
(a)

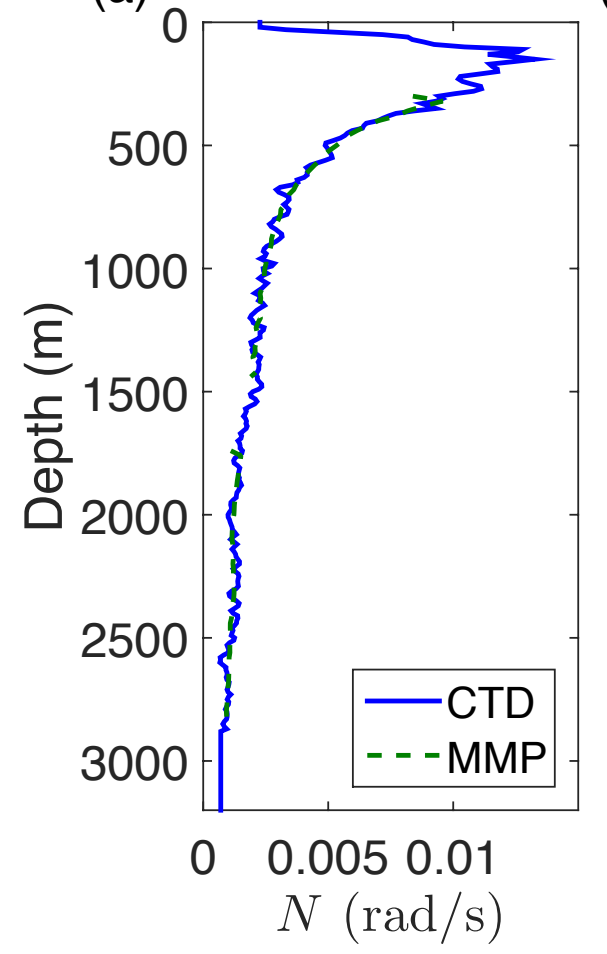

(b)

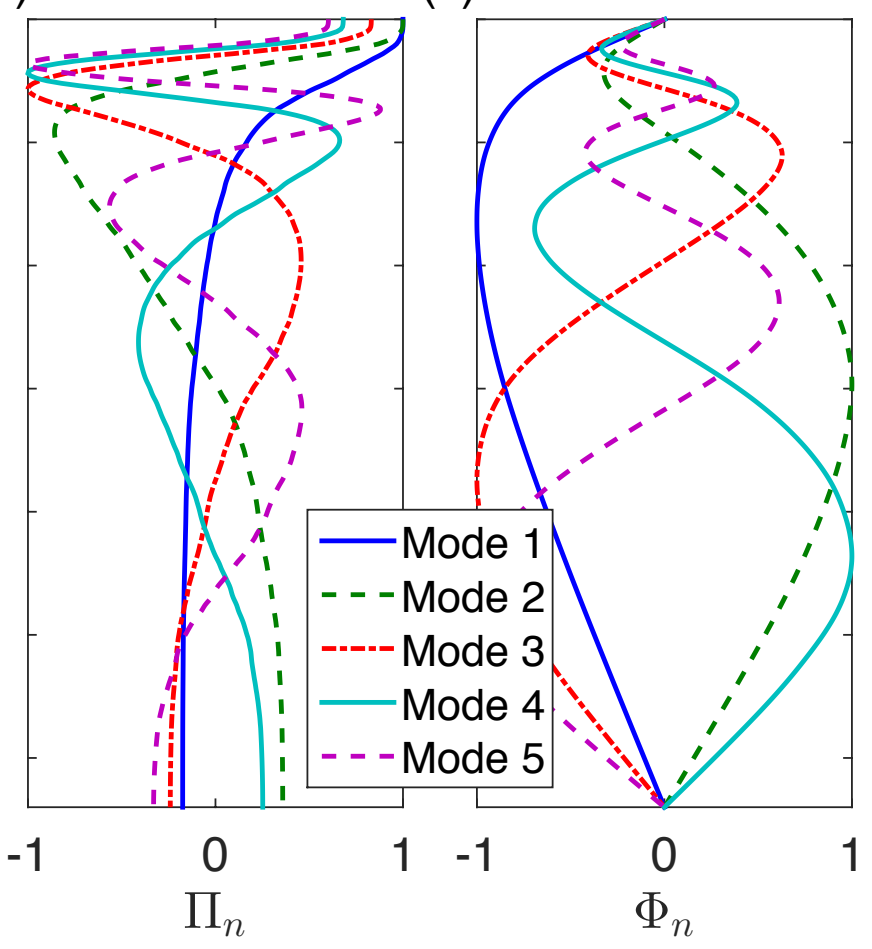

Figure 4-4: Ocean stratification profiles at mooring N: $(a)$ buoyancy frequency profiles (in rad/s) from MMP measurements (green dashed line) and shipboard CTD data (blue line). (b) Normalized vertical structures of the first five baroclinic modes for pressure and horizontal velocity. (c) As in (b), but for vertical displacement and vertical velocity.

$$
c_{p, n}=\frac{\omega}{\sqrt{\omega^{2}-f^{2}}} c_{n}
$$

and

$$
c_{g, n}=\frac{\sqrt{\omega^{2}-f^{2}}}{\omega} c_{n}
$$

where $\omega$ is the tidal frequency $\left(M_{2}\right.$ or $\left.S_{2}\right)$ and $f$ is the inertial frequency (Rainville \& Pinkel, 2006).

The baroclinic horizontal velocity and displacement can be expressed in terms of time-varying modal amplitudes as

$$
\mathbf{u}_{n}^{\prime}(z, t)=\sum_{n=0}^{10} \mathbf{u}_{n}^{\prime}(t) \Pi_{n}(z),
$$


and

$$
\eta_{n}^{\prime}(z, t)=\sum_{n=1}^{10} \eta_{n}^{\prime}(t) \Phi_{n}(z)
$$

where $n$ is the mode number, $\Phi(z)$ and $\Pi(z)$ are the vertical structures of the baroclinic modes for the horizontal velocity and vertical displacement respectively (shown for mooring $\mathrm{N}$ in figures $4-4(b)$ and $4-4(c))$ and $\mathbf{u}_{n}^{\prime}(t)$ and $\eta_{n}^{\prime}(t)$ are the time-varying magnitudes of the baroclinic modes. By least squares modal fitting, $\mathbf{u}_{n}^{\prime}(t)$ and $\eta_{n}^{\prime}(t)$ are extracted from the velocity and displacement profiles Alford, 2003, Nash et al. 2005). Given the excellent coverage of the water column by the MMPs, the first five modes could be resolved. The zeroth-mode $(n=0)$, corresponding to the barotropic solution, is also allowed in the case of the horizontal velocity.

\subsubsection{Pressure, energy, and energy flux}

For each baroclinic mode, the pressure, energy, and energy flux can be independently determined as follows. As in Nash et al. (2005), the baroclinic pressure perturbation, $p_{n}^{\prime}(z, t)$, is calculated from the displacement modal displacement $\eta_{n}^{\prime}(z, t)$ by

$$
p_{n}^{\prime}(z, t)=\rho_{0} \int_{-z}^{0} N^{2}\left(z^{\prime}\right) \eta_{n}^{\prime}\left(z^{\prime}, t\right) d z^{\prime}-p^{\operatorname{surf}}(t) .
$$

From the definition of baroclinic motions, the depth average of the baroclinic pressure perturbations must be zero. That is

$$
p_{n}^{\prime}(H, t)=\rho_{0} \int_{-H}^{0} N^{2}(z) \eta_{n}^{\prime}(z, t) d z-p^{\text {surf }}(t)=0
$$

hence the sea surface pressure, $p^{\text {surf }}(t)$ can be calculated as

$$
p^{\text {surf }}(t)=\rho_{0} \int_{-H}^{0} N^{2}(z) \eta_{n}^{\prime}(z, t) d z
$$

Depth-integrated horizontal kinetic energy and available potential energy are com- 
puted from the above modal fit baroclinic perturbations, $\mathbf{u}_{n}^{\prime}(z, t)$ and $\eta_{n}^{\prime}(z, t)$, by

$$
\mathrm{HKE}_{n}=\frac{\rho_{0}}{2} \int_{-H}^{0}\left\langle\left|\mathbf{u}_{n}^{\prime}(z, t)\right|^{2}\right\rangle d z
$$

and

$$
\mathrm{APE}_{n}=\frac{\rho_{0}}{2} \int_{-H}^{0}\left\langle N^{2}(z) \eta_{n}^{2}(z, t)\right\rangle d z
$$

where the angle brackets indicate the average over one tidal cycle. The total energy $E$ is calculated by

$$
E_{n}=\mathrm{HKE}_{n}+\mathrm{APE}_{n}
$$

The depth-integrated energy flux is computed as the covariance of the modal fit velocity and pressure perturbations by

$$
\mathbf{F}_{n}=\int_{-H}^{0}\left\langle\mathbf{u}_{n}^{\prime}(z, t) p_{n}^{\prime}(z, t)\right\rangle d z .
$$

\subsection{Mooring-observed internal tides}

This section presents the internal tides as observed in the mooring array at moorings $\mathrm{U}, \mathrm{N}$, and $\mathrm{S}$ using the techniques described in the previous section. Of key interest is the manner in which the modal content of the incident internal tide changes as it encounters the Line Islands Ridge. For this reason, the north-south internal tide is studied at moorings $\mathrm{U}, \mathrm{N}$ and $\mathrm{S}$.

\subsubsection{Interntal tide at mooring $\mathrm{U}$}

Figure 4-5 show the time series of the semidiurnal internal tide measured at mooring U. The semidiurnally bandpassed north-south velocity is shown in color, with isopycnal displacements spaced at 100 m overlaid as black lines. As can be seen from the figure, the top and bottom MMPs at mooring $\mathrm{U}$ performed extremely well during the first deployment and measured almost continuously for the full 100 days. Furthermore, the MMPs continued to function well for most of the second deployment 


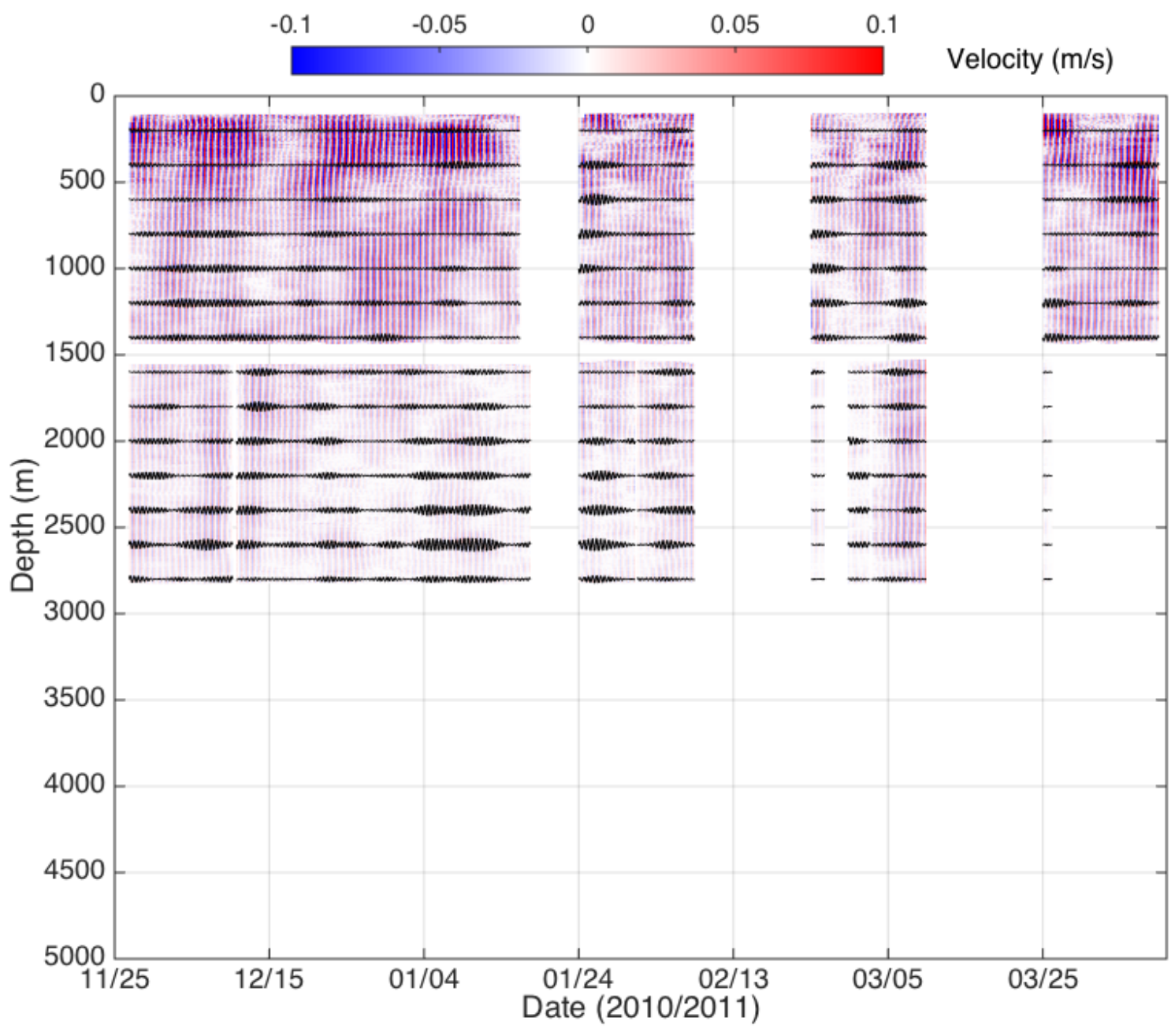

Figure 4-5: Time series of the semidiurnal internal tide at mooring U. Semidiurnally bandpassed north-south velocity (color) along with isopycnal displacement (black lines) with mean spacing every $100 \mathrm{~m}$.

until the final two weeks when the bottom MMP failed for an extended period of time. The MMPs on mooring $\mathrm{U}$ were only able to cover $\sim 53 \%$ of the vertical domain, however, as shown in Chapter 3, the regression method is still able to determine the modal content of the internal tide with a high degree of accuracy. The internal tide is clearly visible at mooring $\mathrm{U}$, over $619 \mathrm{~km}$ away from its primary generation region near French Frigate Shoals and $\sim 100 \mathrm{~km}$ upstream of the Line Islands Ridge, as successive periods of more intense velocities and greater displacements separated by more quiescent periods. 

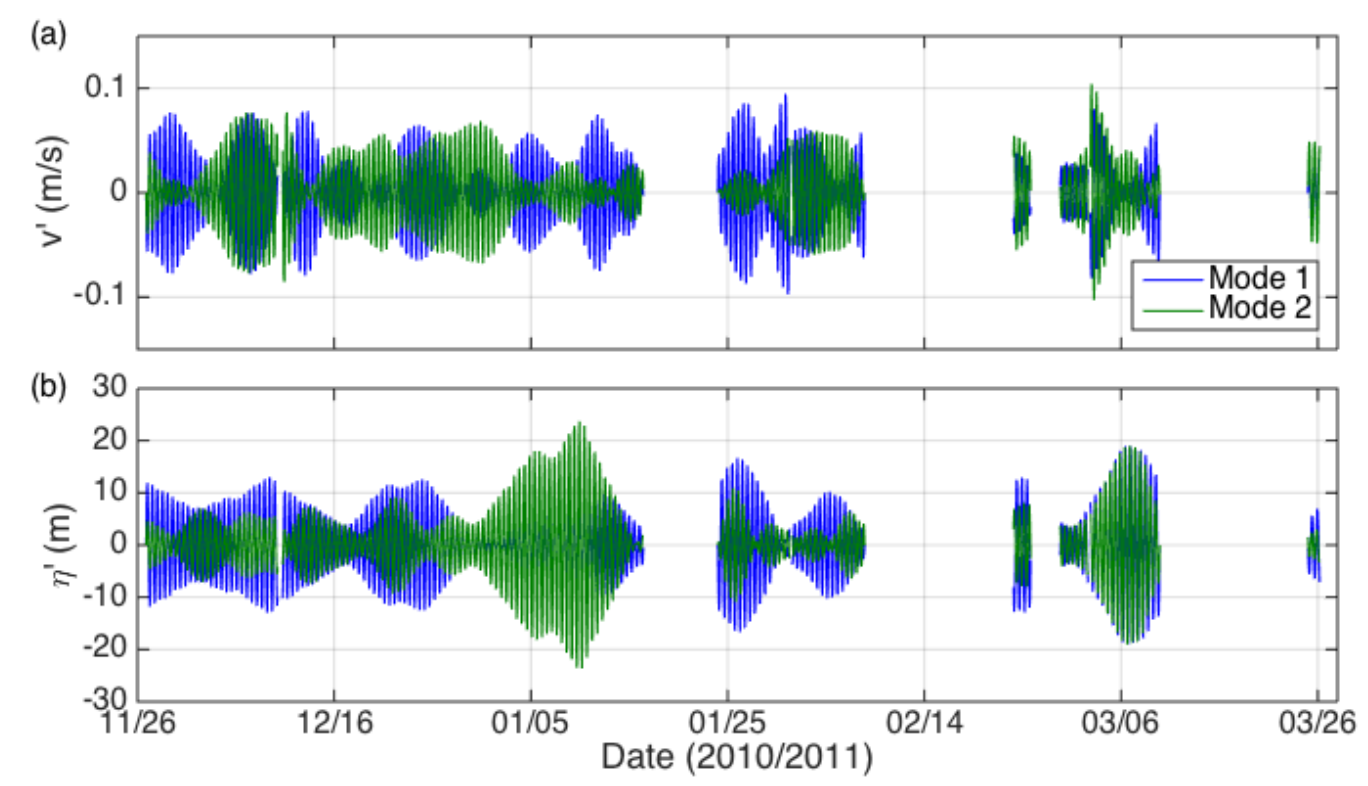

Figure 4-6: Time-varying modal amplitude for the $(a)$ north-south velocity and $(b)$ vertical displacement at mooring $\mathrm{U}$ for modes 1 (blue) and 2 (green)

Figure 4-6(a) presents the time-varying modal amplitude of the north-south velocity at mooring $\mathrm{U}$ for mode 1 in blue and mode 2 in green. Similarly, figure 4-6(b) shows the time-varying modal amplitude of the vertical displacement at mooring $\mathrm{U}$. Modal decomposition indicates a dominance of mode 1 in both the velocity and displacement, with a strong mode-2 signal in the vertical displacement in late December to early January.

The energy flux magnitude for modes 1 (blue), 2 (green), and 3 (red) at mooring

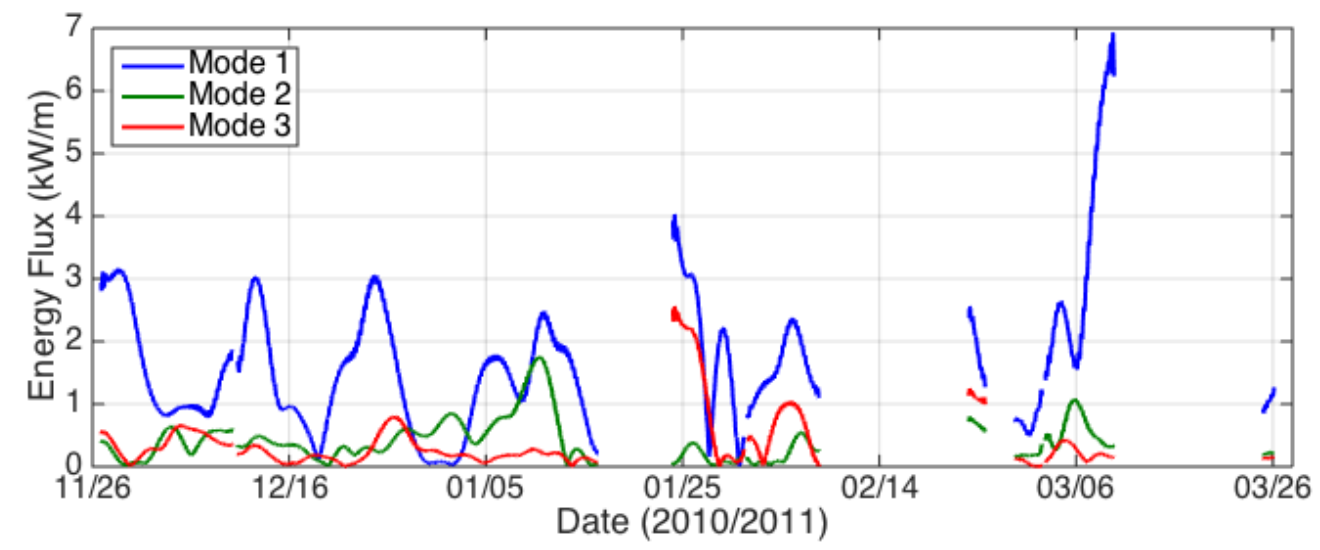

Figure 4-7: Energy flux magnitude in modes 1 (blue), 2 (green), and 3 (red) for mooring $\mathrm{U}$. 
$\mathrm{U}$ is shown in figure 4-7. Despite the stronger mode-2 signal present in the vertical displacement at mooring $\mathrm{U}$, the energy flux at this location is still dominantly mode-1. However, there is a large peak in the mode-1 signal in the beginning of March, with an amplitude of almost $7 \mathrm{~kW} / \mathrm{m}$. Since a peak in the horizontal velocity and vertical displacement at this time is not seen in figure 4-6, this is likely not physical and instead a manifestation of numerical errors in the analysis of such a sparse dataset.

\subsubsection{Interntal tide at mooring $\mathrm{N}$}

As in figure 4-5, the time series of the semidiurnal internal tide measured at mooring $\mathrm{N}$ is shown in figure 4-8. Unlike the mooring $\mathrm{U}$ MMPs, the profilers on mooring $\mathrm{N}$ did not function continuously for the first deployment. Additionally, although they functioned well the during the second deployment, after mid-March the top and bottom moored profilers became unsynchronized. This will pose a problem when determining the modal structure of the internal tide as one MMP does not provide sufficient data for either of the projection algorithm or regression method discussed in Chapter 3 to determine the modal content accurately. Despite such instrument failures, the internal tide is clearly seen as at mooring U. Furthermore, the water column coverage of the stacked MMPs is excellent at mooring N, with over $72 \%$ of the vertical domain measured.

The time-varying modal amplitude of the north-south velocity at mooring $\mathrm{N}$ for mode 1 and 2 is shown in figure 4-9 (a). Modal decomposition indicates a strong dominance of mode 1 in the horizontal velocity signal throughout both of the mooring deployments. Shown in figure 4-9 (b), the time-varying modal amplitude of the vertical displacement, however, has strong mode 1 as well as mode 2 signals.

Figure 4-10 depicts the energy flux magnitude for modes 1 (blue), 2 (green), and 3 (red) at mooring $\mathrm{N}$. Although the vertical displacement at mooring $\mathrm{N}$ has strong signals of both mode 1 and mode 2, the energy flux at this location is still dominantly mode-1. Additionally, unlike mooring $\mathrm{U}$, there the large peak in the mode-1 signal in the beginning of March is absent, further suggesting that the peak in figure 4-7 is not physical. 


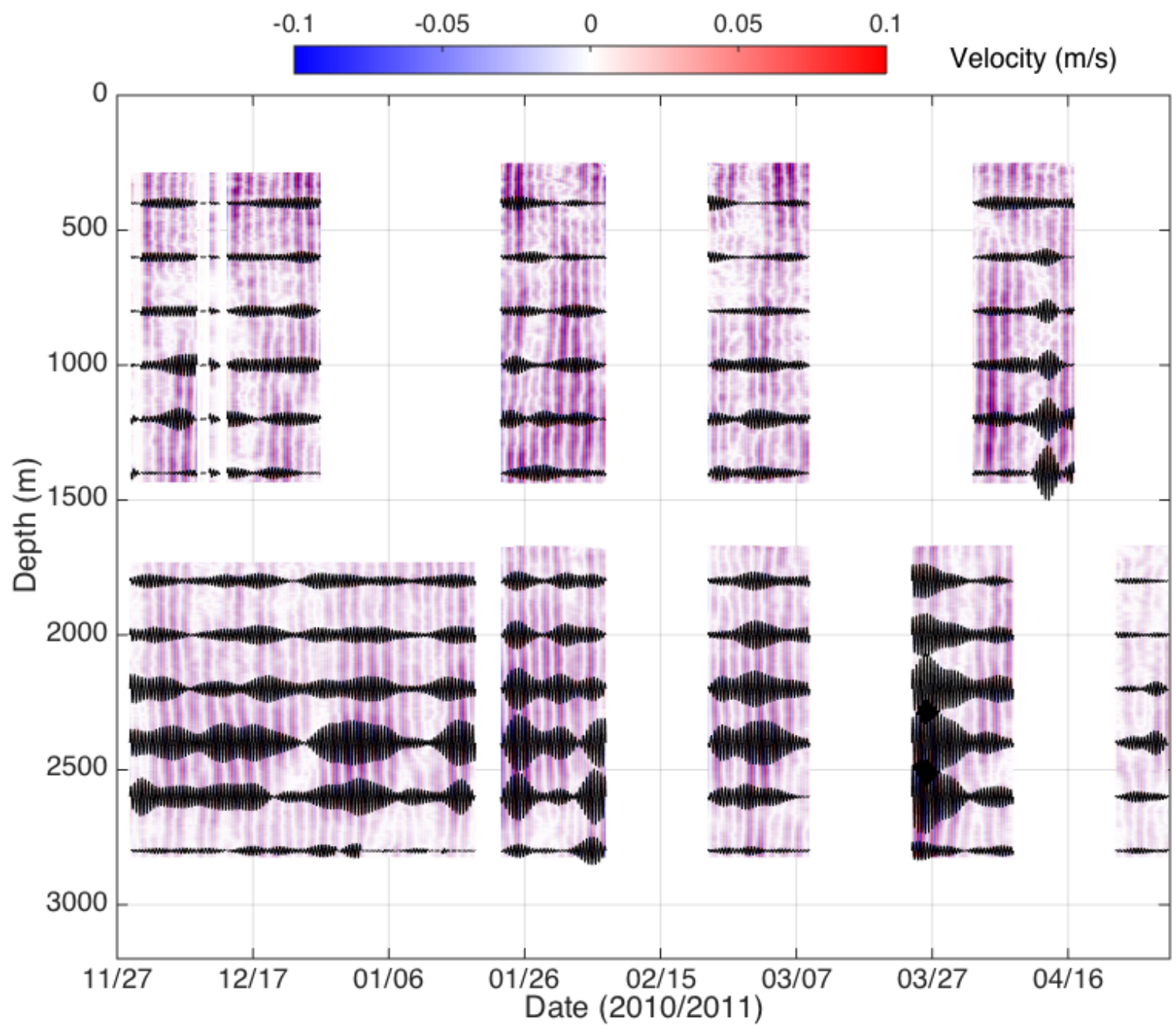

Figure 4-8: Time series of the semidiurnal internal tide at mooring N. Semidiurnally bandpassed north-south velocity (color) along with isopycnal displacement (black lines) with mean spacing every $100 \mathrm{~m}$. 

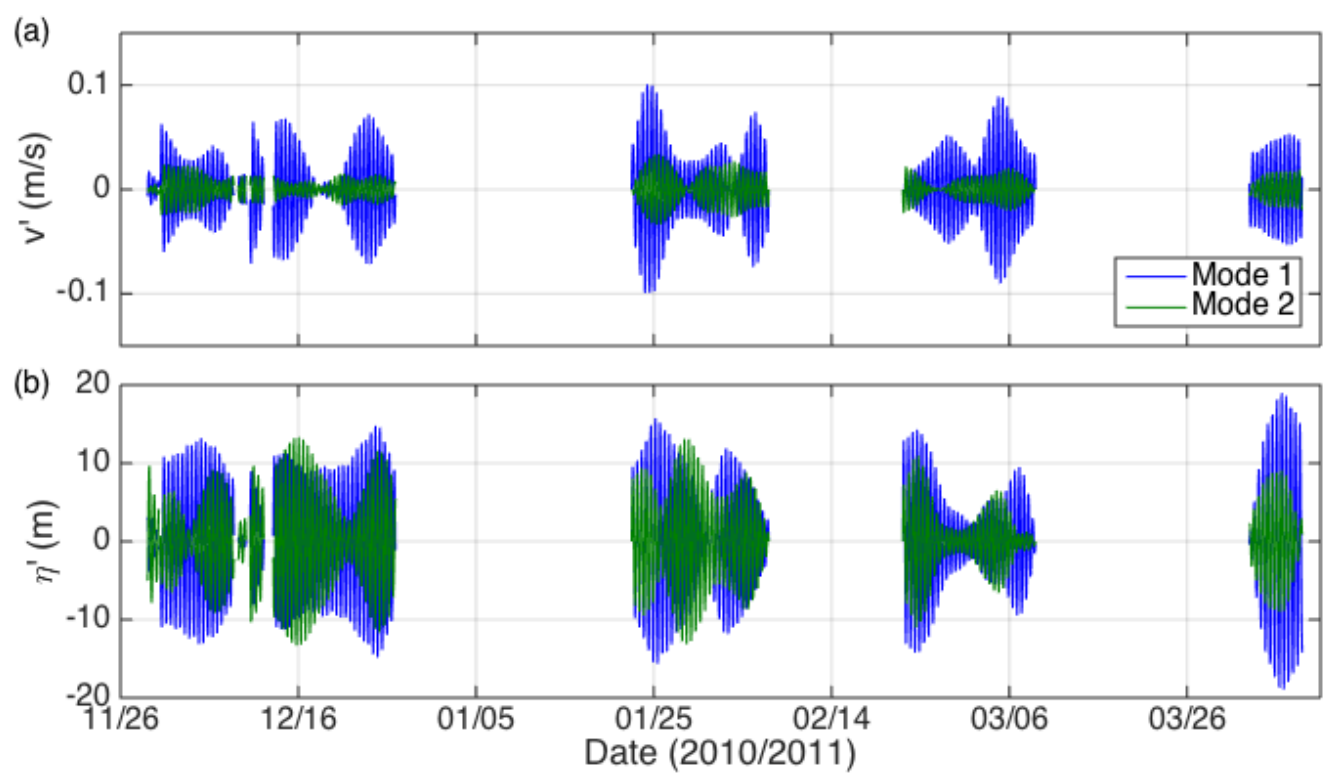

Figure 4-9: Time-varying modal amplitude for the $(a)$ north-south velocity and $(b)$ vertical displacement at mooring $\mathrm{N}$ for modes 1 (blue) and 2 (green).

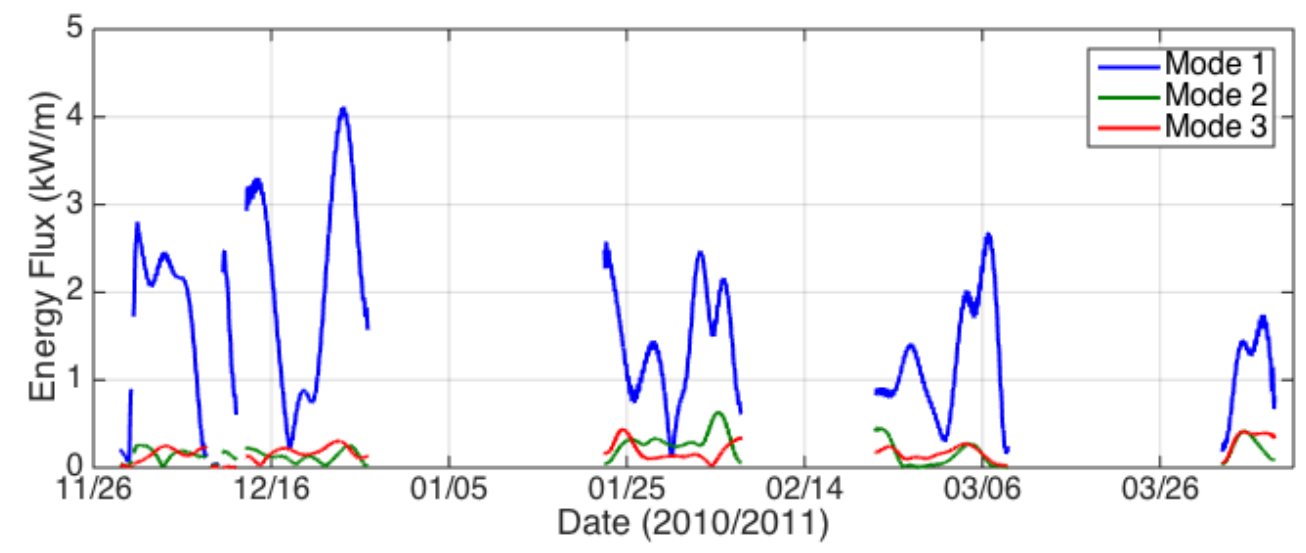

Figure 4-10: Energy flux magnitude in modes 1 (blue), 2 (green), and 3 (red) for mooring $\mathrm{N}$. 


\subsubsection{Interntal tide at mooring $\mathrm{S}$}

At mooring S, to the south and downstream of the Line Islands Ridge, there is again a clear semidiurnal internal tide signal, shown in figure 4-11. As can be seen from the figure, the top MMP failed for the entire first deployment. This resulted in insufficient measurements of the water column for modal decomposition. Therefore, the modal content of the internal tide during the first deployment at mooring $\mathrm{S}$ cannot be determined. However, both top and bottom MMPs functioned extremely well during the second deployment, staying synchronized, collecting measurements for over $90 \%$

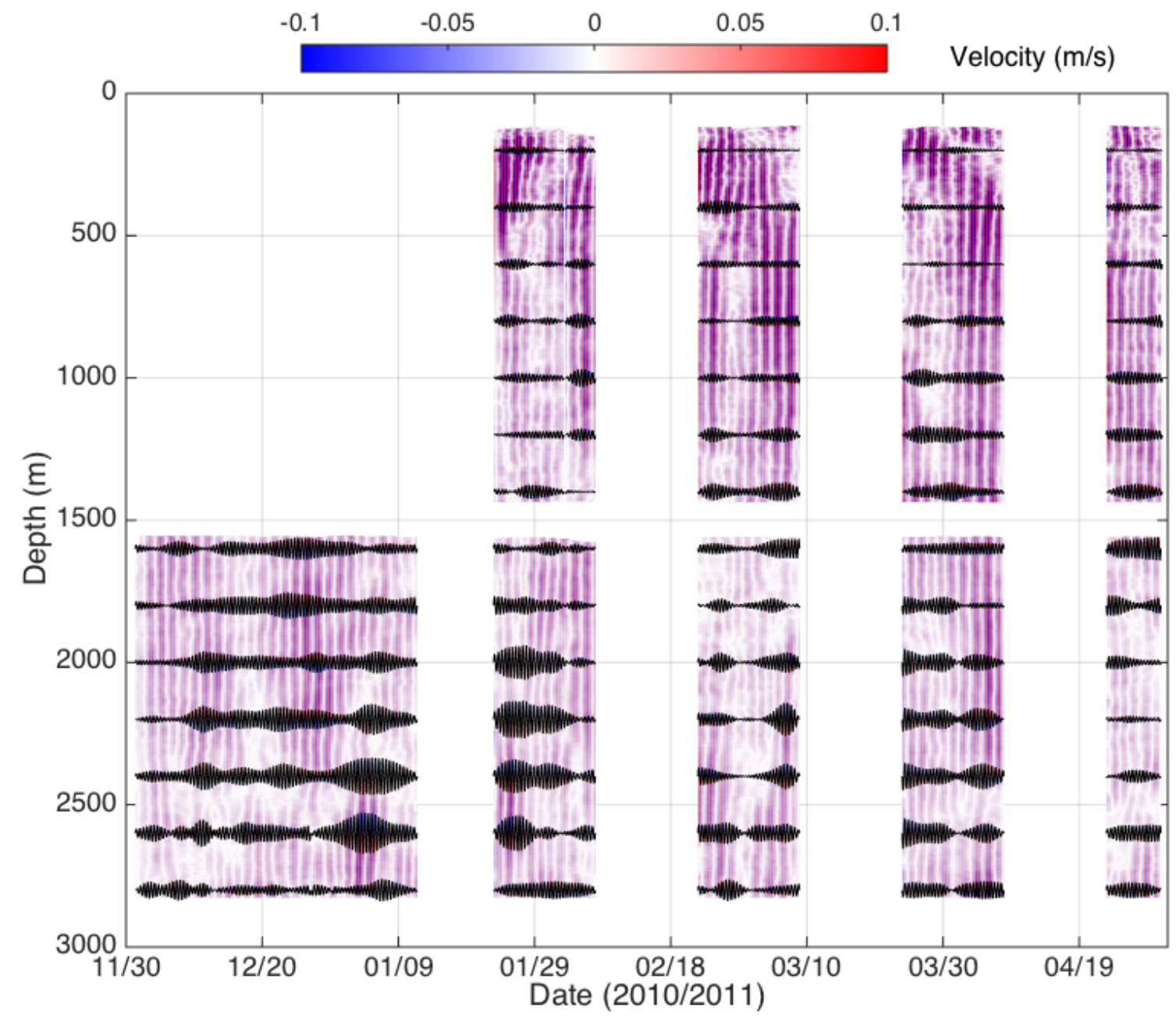

Figure 4-11: Time series of the semidiurnal internal tide at mooring S. Semidiurnally bandpassed north-south velocity (color) along with isopycnal displacement (black lines) with mean spacing every $100 \mathrm{~m}$. 

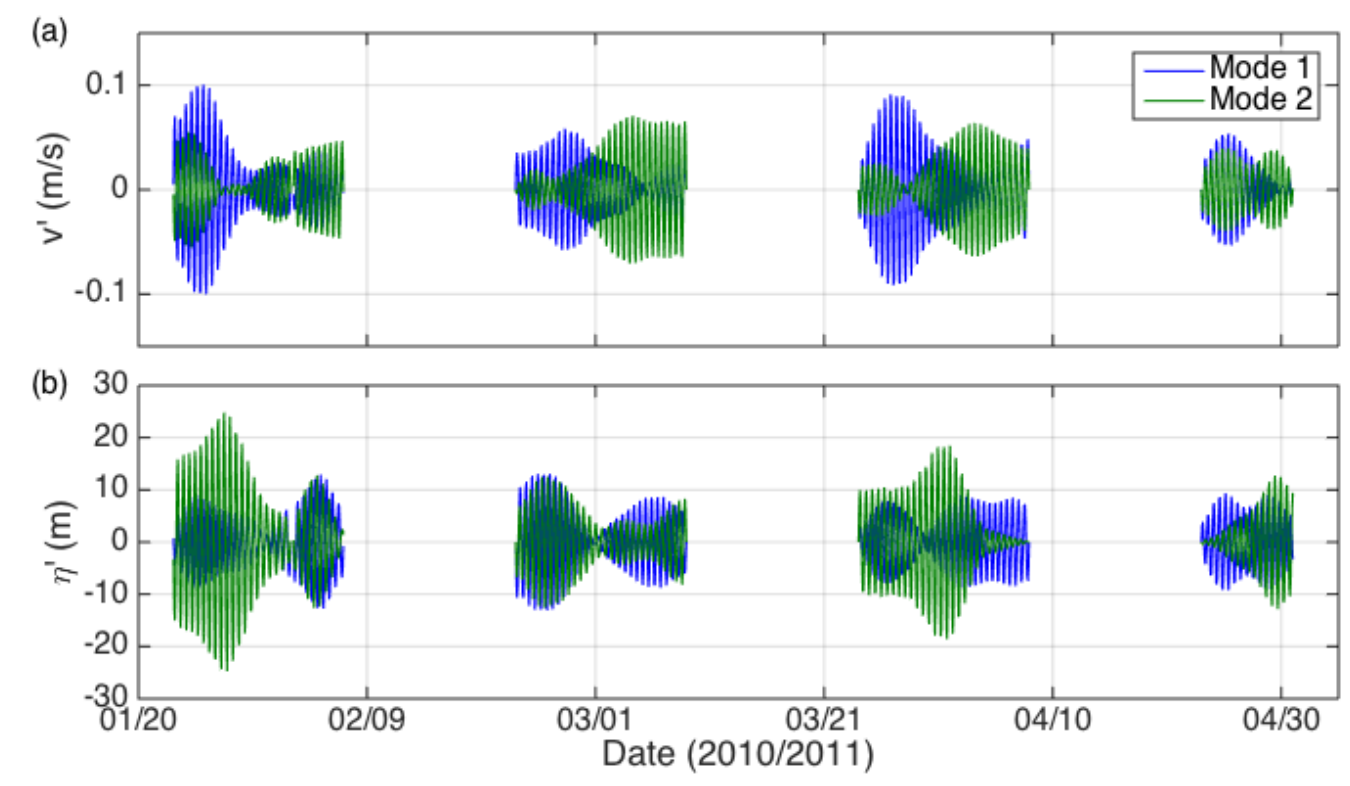

Figure 4-12: Time-varying modal amplitude for the $(a)$ north-south velocity and $(b)$ vertical displacement at mooring $\mathrm{S}$ for modes 1 (blue) and 2 (green).

of the vertical domain, resulting in excellent spatial and temporal coverage for this time period.

Figure 4-12(a) shows the time-varying modal amplitude for the north-south velocity at mooring S. There does not seem to be a clear dominance of either mode 1 or mode 2 in the horizontal velocity signal. Instead, the strength of the two signals alternate; during some periods the horizontal velocity is dominated by mode 1 while at other times it is dominated by mode 2. Figure 4-12(b) shows a similar trend for time-varying modal amplitude of the vertical displacement at mooring $\mathrm{S}$. This is quite different from moorings $\mathrm{U}$ or $\mathrm{N}$ where, although mode-2 was present and at some periods stronger than the mode- 2 signal, the amplitude of the two signals did not alternate as they do at mooring $\mathrm{S}$.

The energy flux magnitude in modes 1, 2 and 3 at mooring S are shown in figure 413. Unlike the semidiurnal internal tide at mooring $\mathrm{U}$ and $\mathrm{N}$, at mooring $\mathrm{S}$ the energy flux, while predominantly mode 1 , is also characterized by large amounts energy flux in mode 2. There are periods during which the energy flux in mode 2 is on the order of that in mode 1. For instance, in the last week of January, the energy flux in mode 2 is more than $50 \%$ of the energy flux in mode 1 . Additionally, at times, such as the end 


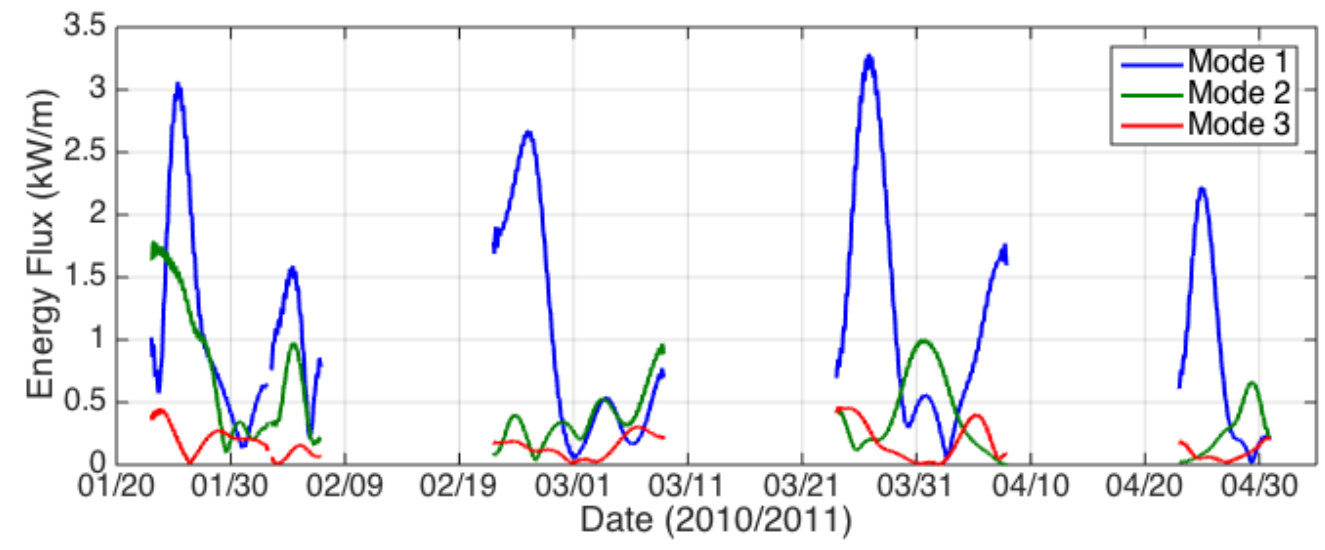

Figure 4-13: Energy flux magnitude in modes 1 (blue), 2 (green), and 3 (red) for mooring S.

of March, the energy flux in mode 2 is greater than that of mode 1 . This suggests that the modal content of the semidiurnal internal tide has increased amounts of mode 2 after encountering the Line Islands Ridge.

\subsubsection{Variations between moorings}

The modal partition of energy is shown in figure 4-14(a). As seen in the figure, the energy of the semidiurnal internal tide at mooring $U$ is quite complicated, with a large amount of energy in mode 3, while at mooring $\mathrm{N}$ the signal is dominated by mode 1. Mooring S stands out for its reduced mode-1 contribution and strong mode-2 contribution of energy, suggestive of topographic scattering.

Figure 4-14(b) demonstrates the modal partition of energy flux at the various moorings of the EXITS cruise. While mooring $U$ shows a dominance of mode-3 energy, the energy flux continues to have a strong mode-1 dominance. This lowmode internal tide is further seen at mooring $\mathrm{N}$ where the energy flux of the mode- 1 internal tide is the strongest. The trend, however, is not sustained at mooring $\mathrm{S}$, where the energy flux in mode 1 is decreased and an increase in the mode- 2 energy flux is apparent. This result is consistent with analysis of satellite altimetry of the internal tide at the Line Islands Ridge (Johnston et al., 2003).

The partitioning between HKE and APE, shown in figure 4-14(a), also varies between the moorings. For a free-propagating internal tide, HKE always exceeds APE 
(a)

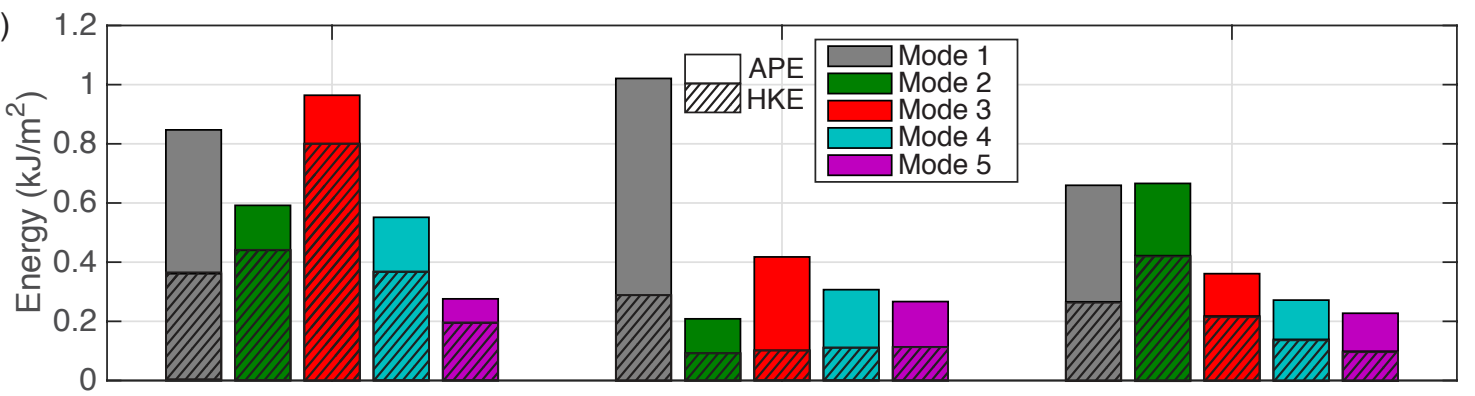

(b)

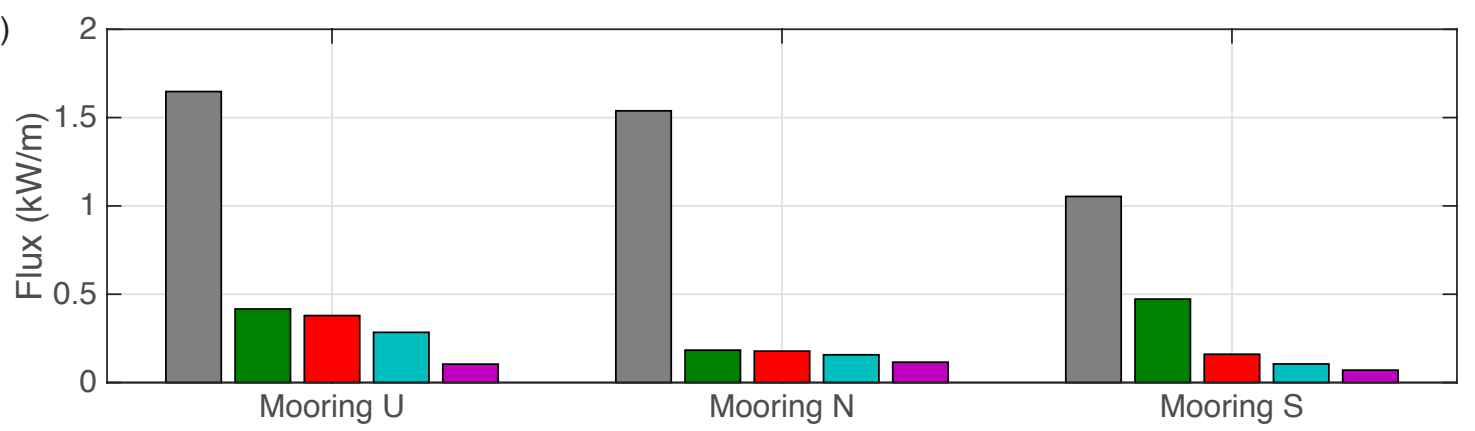

Figure 4-14: Modal partition of $(a)$ energy and $(b)$ energy flux of the semidiurnal internal tide measured at moorings $\mathrm{U}, \mathrm{N}$, and $\mathrm{S}$. In $(a)$, HKE is distinguished with a hatching pattern.

in a latitude-dependent ratio ranging from 1.2267 at mooring $\mathrm{U}$ to 1.2 at mooring $\mathrm{S}$, following

$$
\frac{\mathrm{HKE}}{\mathrm{APE}}=\frac{\omega^{2}+f^{2}}{\omega^{2}-f^{2}}
$$

where $\omega$ is the tidal frequency and $f$ is the internal frequency. Unlike at mooring $\mathrm{U}$ and $\mathrm{S}$, the observed APE at mooring $\mathrm{N}$ exceeds the HKE, implying standing-wave patterns (Alford \& Zhao, 2007, Zhao \& Alford, 2009).

\subsection{Uncertainties}

Extensive error analysis was done by Zhao et al. (2010) as part of the Internal Waves Across the Pacific (IWAP) study examining the northward propagation of the internal tide generated by Hawaiian Ridge, the same generation site of the southward propagating internal tide that has been observed to scatter at the Line Islands Ridge. IWAP included six moorings each equipped with a McLane moored profiler measur- 
ing data in the upper $1400 \mathrm{~m}$ of the total $5000 \mathrm{~m}$ ocean depth. In addition, a single current meter collected point measurements at $3000 \mathrm{~m}$.

Following Nash et al. (2005), the magnitude of the effect of the imperfect vertical resolution in generating errors in the modal partitioning of the internal tidal signals was assessed with Monte Carlo simulations by running a synthetically generated wave field comprised of a superposition of 30 modes past the mooring geometry. The results of their analyses found, similar to Rainville \& Pinkel (2006), mode-1 displacement was reliably estimated without deep data, where as the single point measurements at $3000 \mathrm{~m}$ were critical to constraining the fits for displacement modes $\geq 2$ and all velocity modes. Mode-1 fluxes were unreliable when the deep data were excluded from calculation but were otherwise insensitive to weighting. With the weighting of the single point measurement data at $3000 \mathrm{~m}$, corresponding fractional standard errors for each uncorrelated estimate were $8 \%, 30 \%$, and $50 \%$ for the first three modes.

Considering that the mooring geometry of the EXITS cruise results in much greater coverage of the water column than IWAP, over $90 \%$ in the case of mooring $\mathrm{S}$, the errors in modal decomposition of the wave field is expected to be much less than that of the IWAP study. Additionally, the horizontal velocity, vertical displacement, and energy flux magnitudes at all are moorings are in good agreement with previous observations in this region as reported by Zhao et al. (2010) which measured velocities $O(0.1) \mathrm{m} / \mathrm{s}$, displacements $O(15) \mathrm{m}$, and energy flux magnitudes $O(4) \mathrm{kW} / \mathrm{m}$. Furthermore, these values are in good agreement with results of three-dimensional numerical simulations and altimetric observations by Johnston et al. (2003) of the Line Islands Ridge which predict vertical displacements $O(25) \mathrm{m}$ and energy flux magnitudes $O(2) \mathrm{kW} / \mathrm{m}$.

Further work needs to be done following the Monte Carlo simulations of Zhao et al. (2010) and Nash et al. (2005) to determine the effect of imperfect vertical resolution in generating errors in the modal decomposition. Additional investigation should be done to determine the influence of data from the current meters at the top and bottom of each mooring on the modal decomposition of the wave field. This will likely have the most effect at mooring $\mathrm{U}$, where only $\sim 51 \%$ of the water column was sampled. 


\subsection{Conclusion}

In summary, these observations indicate a rich time-variable multimodal internal tide field. The modal content of the internal tide upstream of the Line Islands Ridge at moorings $\mathrm{U}$ and $\mathrm{N}$ is dominated by mode 1 , as seen in the time varying modal amplitudes of the horizontal velocity and vertical displacement as well as the energy flux at both locations. On the other hand, the modal content of the internal tide downstream at mooring $\mathrm{S}$ has a weaker mode-1 signal and a stronger mode-2 signal, seen in the modal amplitudes of the velocity and displacement as well as the energy flux. Furthermore, the modal partition of the energy flux at mooring $\mathrm{S}$ has a decreased amount of mode 1 and a significant increase in mode 2 than at moorings $\mathrm{U}$ and $\mathrm{N}$, although it is still mode-1 dominant.

However, the Line Islands Ridge is a region with complex bathymetry and the mooring locations for the EXITS cruise did not fall upon the same transect. Therefore, the far-field internal tide seen at mooring $U$ may have been changed by topographic features before it reached mooring $\mathrm{N}$. Additionally, the feature between mooring $\mathrm{N}$ and S was highly three-dimensional. This, along with the implication of standingwave patterns at mooring $\mathrm{N}$, suggest complicated topographic and internal wave interactions are taking place at both mooring $\mathrm{N}$ and $\mathrm{S}$ which may not be indicative of the far-field internal tide.

This is further evidenced by primitive equation model simulations of the Line Islands Ridge of the $M_{2}$ baroclinic energy flux, shown in figure 4-15 (based on Carter et al. (2008) with data from conversations with Dr. Glenn Carter). From the figure, it is clear that the internal tide propagating from French Frigate Shoals is emanates in three dimensions, before a large southward propagating beam encounters the Line Islands Ridge. At the location of the EXITS study, the energy flux in mode 1 is seen to decrease downstream of the ridge where the mode-2 energy flux increases, however it does so slightly farther south of where mooring $\mathrm{S}$ was located. 
(a)

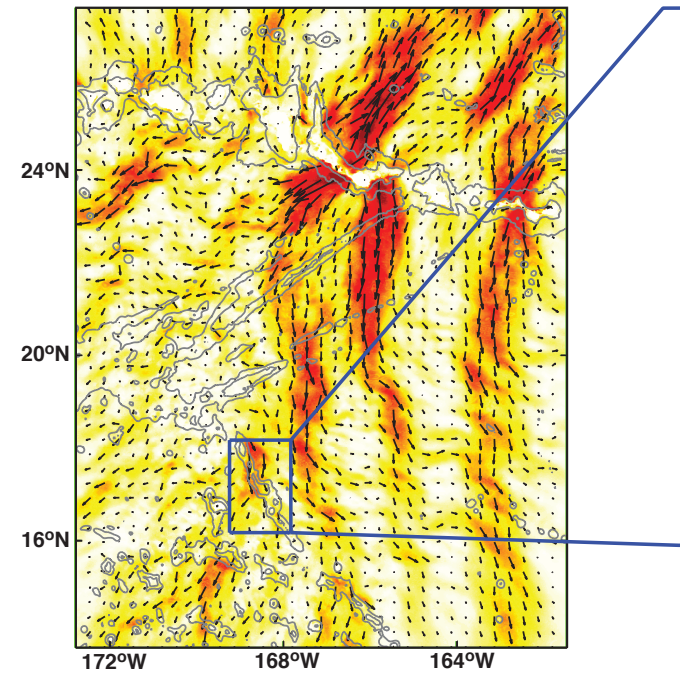

(b)

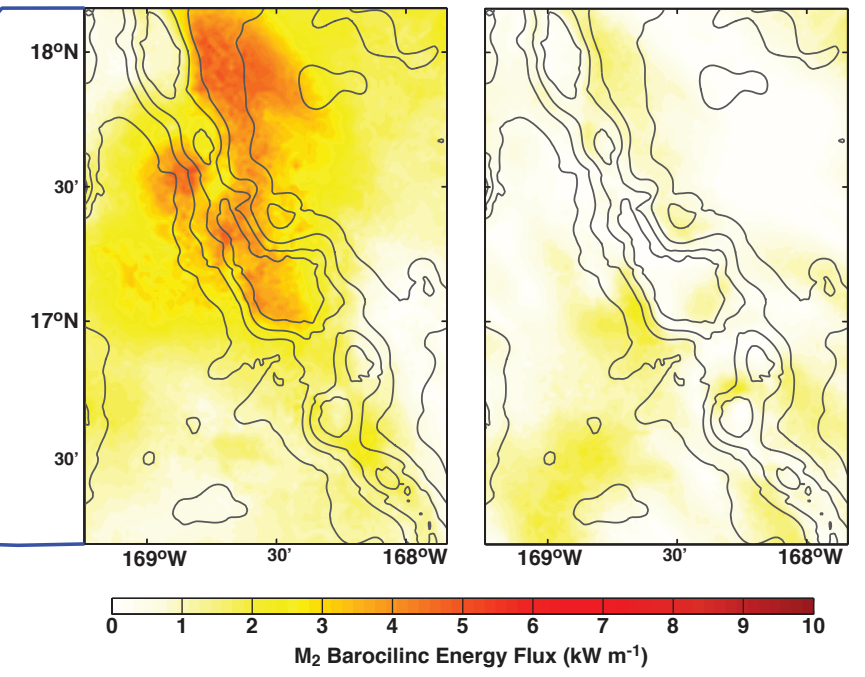

Figure 4-15: The (a) total energy flux, (b) mode-1 energy flux, and $(c)$ mode-2 energy flux at the Line Islands Ridge as predicted by a high-resolution primitive equation model simulation. In $(a)$ every eight depth-integrated $M_{2}$ baroclinic energy flux vectors in each direction has been plotted. The underlying color gives the flux magnitude. Plots $(b)$ and $(c)$ zoom in on the EXITS study region. Contour interval is $1000 \mathrm{~m}$. Based on Carter et al. (2008) with data from conversations with Dr. Glenn Carter. 


\section{Chapter 5}

\section{Conclusions}

Internal tides are key to understanding mixing processes in the ocean. They draw energy from the barotropic tide and their eventual dissipation drives ocean circulation. Specifically, the majority of the energy of an internal tide is contained in low-modes, which have been observed to propagate over a thousand kilometers away, while highmode internal tides dissipate locally. This thesis investigates the scattering of internal tides by deep-ocean topography as a method of redistributing the energy of low-mode internal tides to higher modes that are more susceptible to energy dissipation via small-scale processes resulting in internal-tide driven mixing at the scattering site. Analytical methods were used to estimate the internal tide generation and scattering characteristics of the Line Islands Ridge, the location of the first field observation of internal tide scattering from the EXperiment on Internal Tide Scattering (EXITS). Additionally, theory was developed to determine the modal content of internal wave fields for the goal of being able to reliably distinguish the modal content of a wave field given limited data in the vertical domain. Finally, insights from these analytical studies and modal decomposition methods were used to analyze field observations from EXITS for evidence of internal tide scattering.

The Green function approach, a theoretical frame work for modeling the twodimensional interaction of internal waves with topography, was described in Chapter 2 . The theory was utilized to predict both the generated and scattered wave fields at two transects of the Line Islands Ridge that were the subject of investigation in 
EXITS. The results from this chapter were used to aid in the analysis of the observational field data from the EXITS study, detailed in Chapter 4 . The topography at the location considered was shown to be efficient at converting a significant fraction of the incident mode-1 energy flux to higher modes, thus serving as a strong source of internal tide dissipation as the higher modes are more susceptible to instability and breaking.

Chapter 3 presented an algorithm to decompose two-dimensional wave fields in constant depth into their constitutive modes. An analytical expression for the modal content was derived for the case of incomplete vertical domain data, which is common in oceanographic field studies as well as laboratory experiments. The performance of the modal decomposition technique was compared against a regression technique, the current standard method of modal decomposition of oceanographic field data, by applying both methods to an idealized wave field with varying amounts of image loss. The chapter concluded with a comparison of the two techniques using a wave field generated using Green function theory at one of the transects studied in EXITS at the Line Islands Ridge with image loss consistent with a field experiment using two stacked moored profilers, as was the case in EXITS.

The EXITS cruise, the first observational study to investigate internal tide scattering, was discussed in Chapter 4. The data and methods of gathering measurements using two stacked moored profilers from moorings upstream and downstream of the Line Islands Ridge was discussed. The data was processed for evidence of the semidiurnal internal tide and analyzed to determine the energy and energy flux of the internal tide before and after encountering the topographic feature. Results indicated that the internal tide content upstream of the ridge was dominated by mode 1 , while downstream of the ridge the internal tide had equal, and sometimes greater, contributions from mode 2 , suggestive of internal tide scattering.

Further work will include the determination of the number of modes required by the projection method described in Chapter 3 to ensure reliable convergence of the algorithm without requiring knowledge of the modal content of the wave field a priori. Additional work will focus on the application of this modal decomposition method to 
wave fields generated and scattered by various topographies as predicted by the Green function approach, described in Chapter 2. Particularly, the use of local topographic depth as opposed to far-field depth (and corresponding image loss of the velocity field below the topography) in the accurate determination of modal content will be studied for topographies of varying criticality and height. Additional work will be done to explore for which classes of topographies the modal content of the internal tide above the topography is in good quantitative agreement with the far-field internal tide. Moreover, the validity in utilizing flat-bottom modes for the modal decomposition of wave fields above these topographic features will be investigated with the aim of determining when and how flat-bottom modes may be implemented to determine the modal content of an internal wave field. 


\section{Bibliography}

M. J. Alexander, J. H. Richter, \& B. R. Sutherland. Generation and trapping of gravity waves from convection with comparison to parameterization. J. Atmos. Sci., 63:2963-2977, 2006.

M. H. Alford. Redistribution of energy available for ocean mixing by long-range propagation of internal waves. Nature, 21:159-163, 2003.

M. H. Alford \& Z. Zhao. Global patterns of low-mode internal-wave propagation. Part I: Energy and energy flux. J. Phys. Oceanogr., 37:18291848, 2007.

M. H. Alford, J. A. MacKinnon, Z. Zhao, R. Pinkel, J. Klymak, \& T. Peacock. Internal waves across the pacific. Geophys. Res. Lett., 34:L24601, 2007.

Matthew H. Alford. Sustained, Full-Water-Column Observations of Internal Waves and Mixing near Mendocino Escarpment. J. Phys. Oceanogr., 40(12):2643-2660, 2010.

N. J. Balmforth \& T. Peacock. Tidal conversion by supercritical topography. J. Phys. Oceanogr., 39:1965-1974, 2009.

G. S. Carter, M. A. Merrifield, J. M. Becker, K. Katsumata, M. C. Gregg, D. S. Luther, M. D. Levine, T. J. Boyd, \& Y. L. Firing. Energetics of $\mathrm{M}_{2}$ barotropicto-baroclinic tidal conversion at the Hawaiian islands. J. Phys. Oceanogr., 38: 2205-2223, 2008.

K. W. Doherty, D. E. Frye, S. P. Liberatore, \& J. M. Toole. A Moored Profiling Instrument. J. Atmos. Oceanic Technol., 16(1989):1816-1830, 1999.

B. D. Dushaw, B. M. Howe, Cornuelle B. D., P. F. Worcester, \& D. S. Luther. Barotropic and baroclinic tides in the central north pacific ocean determined from long-range reciprocal acoustic transmissions. J. Phys. Oceanogr., 25:631-647, 1995.

P. Echeverri. Internal Tide Generation by Tall Ocean Ridges. PhD thesis, Massachusetts Institute of Technology, 2009.

P. Echeverri \& T. Peacock. Internal tide generation by arbitrary two-dimensional topography. J. Fluid Mech., 659:247-266, 2010. 
P. Echeverri, M. R. Flynn, K. B. Winters, \& T. Peacock. Low-mode internal tide generation by topography: An experimental and numerical investigation. J. Fluid Mech., 636:91-108, 2009.

G. D. Egbert \& R. D. Ray. Estimates of $\mathrm{M}_{2}$ tidal dissipation from TOPEX/POSEIDON altimeter data. J. Geophys. Res., 106:22475-22502, 2001.

C. Garrett \& E. Kunze. Internal tide generation in the deep ocean. Annu. Rev. Fluid Mech., 39:57-87, 2007.

A. E. Gill. Atmosphere-Ocean Dynamics. Academic Press, New York, 662pp., 1982.

C. O. Hines. Internal atmospheric gravity waves at ionospheric heights. Can. J. Phys., 38(11):1441-1481, 1960.

T. M. S. Johnston \& M. A. Merrifield. Internal tide scattering at seamounts, ridges and islands. J. Geophys. Res., 108(C6):3180, 2003.

T. M. S. Johnston, M. A. Merrifield, \& P. E. Holloway. Internal tide scattering at the Line Islands Ridge. J. Geophys. Res., 108(C11):3365, 2003.

T. M. S. Johnston, D. L. Rudnick, M. H. Alford, A. Pickering, \& H. L. Simmons. Internal tidal energy fluxes in the South China Sea from density and velocity measurements by gliders. J. Geophys. Res., 118(8):3939-3949, 2013.

S. M. Kelly, N. L. Jones, \& J. D. Nash. A coupled model for Laplace's tidal equations in a fluid with one horizontal dimension and variable depth. J. Phys. Oceanogr., 43(8):1780-1797, 2013.

E. Kunze \& S. G. Llewellyn Smith. The role of small-scale topography in turbulent mixing of the global ocean. Oceanography, 17:55-64, 2004.

P. H. LeBlond \& L. A. Mysak. Waves in the Ocean. Elsevier Scientific Pub. Co., New York, NY, 1978.

J. R. Ledwell, E. T. Montgomery, K. L. Polzin, L. C. St. Laurent, R. W. Schmitt, \& J. M. Toole. Evidence for enhanced mixing over rough topography in the abyssal ocean. Nature, 403:179-182.

C. M. Lee, E. Kunze, T. B. Sanford, J. D. Nash, M. A. Merrifield, \& P. E. Holloway. Internal tides and turbulence along the $3000-\mathrm{m}$ isobath of the Hawaiian ridge. $J$. Phys. Oceanogr., 36:1165-1183, 2006.

S. Legg. Scattering of low-mode internal waves at finite isolated topography. J. Phys. Oceanogr., 44:359-383, 2014.

S. G. Llewellyn Smith \& W. R. Young. Conversion of the barotropic tide. J. Phys. Oceanogr., 32:1554-1566, 2002. 
S. G. Llewellyn Smith \& W. R. Young. Tidal conversion at a very steep ridge. J. Fluid Mech., 495:175-191, 2003.

J. A. MacKinnon \& K. B. Winters. Subtropical catastrophe: Significant loss of lowmode tidal energy at 28.9 . Geophys. Res. Lett., 32:L15605, 2005.

M. Mathur, G. S. Carter, \& T. Peacock. Topographic scattering of the low-mode internal tide in the deep ocean. J. Geophys. Res., 119(4):2165-2182, 2014.

M. J. Mercier, M. Mathur, M. N. Haji, M. Buijsman, S. J. Ghaemsaidi, P. Echeverri, \& T. Peacock. iTides: A semi-analytical method for the calculation of internal tide generation and scattering by two-dimensional topography. Comput. Geosci., in prep.

M. A. Merrifield \& P. E. Holloway. Model estimates of M2 internal tide energetics at the Hawaiian ridge. J. Geophys. Res., 107(C8):3179-3190, 2002.

P. Müller \& X. Liu. Scattering of internal waves at finite topography in two dimensions. Part I: Theory and case studies. J. Phys. Oceanogr., 30:532-549, 2000.

P. Müller \& N. Xu. Scattering of oceanic internal gravity waves off random bottom toporgaphy. J. Phys. Oceanogr., 22:474-488, 1992.

W. Munk \& C. Wunsch. Abyssal recipes II: Energetics of tidal and wind mixing. Deep-Sea Res., 45:1977-2010, 1998.

J. D. Nash, M. H. Alford, \& E. Kunze. Estimating internal wave energy fluxes in the ocean. J. Atmos. Oceanic Technol., 22(10):1551-1570, 2005.

Y. Niwa \& T. Hibiya. Numerical study of the spatial distribution of the $\mathrm{M}_{2}$ internal tide in the Pacific Ocean. J. Geophys. Res., 106(C10):22441-22449, 2001.

F. Pétrélis, S. G. Llewellyn Smith, \& W. R. Young. Tidal conversion at a submarine ridge. J. Phys. Oceanogr, 36:1053-1071, 2006.

K. L. Polzin. Idealized solutions for the energy balance of the finescale internal wave field. J. Phys. Oceanogr., 34:213-246, 2004.

K. L. Polzin, J. M. Toole, J. R. Ledwell, \& R. W. Schmitt. Spatial variability of turbulent mixing in the abyssal ocean. Science, 276:93-96, 1997.

L. Rainville \& R. Pinkel. Propagation of low-mode internal waves through the ocean. J. Phys. Oceanogr., 36:1220-1236, 2006.

R. D. Ray \& D. E. Cartwright. Estimates of internal tide energy fluxes from TOPEX/Poseidon altimetry: Central North Pacific. Geophys. Res. Lett., 28:12591262, 2001.

R. D. Ray \& G. T. Mitchum. Surface manifestation of internal tides in the deep ocean: Observations from altimetry and island gauges. Prog. Oceanogr., 40:135-162, 1997. 
R. M. Robinson. The effects of a barrier on internal waves. Deep Sea Res., 16:421-429, 1969.

D. L. Rudnick, T. J. Boyd, R. E. Brainard, G. S. Carter, G. D. Egbert, M. C. Gregg, P. E. Holloway, J. M. Klymak, E. Kunze, C. M. Lee, M. D. Levine, D. S. Luther, J. P. Martin, M. A. Merrifield, J. N. Moum, J. D. Nash, R. Pinkel, L. Rainville, \& T. B. Sanford. From tides to mixing along the Hawaiian ridge. Science, 301: 355-357, 2003.

S. J. Saidi. Experimental Investigation of $2 D$ and 3D Internal Wave Fields. Master's thesis, Massachusetts Institute of Technology, 2011.

L. C. St. Laurent \& C. Garrett. The role of internal tides in mixing the deep ocean. J. Phys. Oceanogr., 32:2882-2899, 2002.

L. C. St. Laurent \& J. D. Nash. An examination of the radiative and dissipative properties of the internal tides. Deep-Sea Res. II, 51:3029-3042, 2004.

C. Staquet \& J. Sommeria. Internal gravity waves: From instabilities to turbulence. Annu. Rev. Fluid Mech., 34:559593, 2002.

Y. K. Tsang, W. R. Young, \& N. J. Balmforth. Near-inertial parametric subharmonic instability. J. Fluid Mech., 607:2549, 2008.

C. Wunsch \& R. Ferrari. Vertical mixing, energy, and the general circulation of the oceans. Annu. Rev. Fluid Mech., 36:281-314, 2004.

Z. Zhao \& M. H. Alford. New altimetric estimates of Mode- $1 \mathrm{M}_{2}$ internal tides in the Central North Pacific Ocean. J. Phys. Oceanogr., 39(7):1669-1684, 2009.

Z. Zhao, M. H. Alford, J. MacKinnon, \& R. Pinkel. Long-Range Propagation of the Semidiurnal Internal Tide from the Hawaiian Ridge. J. Phy. Oceanogr., 40(4): 713-736, 2010.

Z. Zhao, M. H. Alford, J. Girton, T. M. Johnston, \& G. Carter. Internal tides around the Hawaiian Ridge estimated from multisatellite altimetry. J. Geophys. Res., 116: C12039, 2011.

Z. Zhao, M. H. Alford, \& J. B. Girton. Mapping low-mode internal tides from multisatellite altimetry. Oceanography, 25(2):42-51, 2012. 